\title{
WestVirginiaUniversity
}

THE RESEARCH REPOSITORY @ WVU

Graduate Theses, Dissertations, and Problem Reports

1998

\section{Impedance effects of metallic ground plane on the CTHA antenna}

Tao Guan

West Virginia University

Follow this and additional works at: https://researchrepository.wvu.edu/etd

\section{Recommended Citation}

Guan, Tao, "Impedance effects of metallic ground plane on the CTHA antenna" (1998). Graduate Theses, Dissertations, and Problem Reports. 910.

https://researchrepository.wvu.edu/etd/910

This Thesis is protected by copyright and/or related rights. It has been brought to you by the The Research Repository @ WVU with permission from the rights-holder(s). You are free to use this Thesis in any way that is permitted by the copyright and related rights legislation that applies to your use. For other uses you must obtain permission from the rights-holder(s) directly, unless additional rights are indicated by a Creative Commons license in the record and/ or on the work itself. This Thesis has been accepted for inclusion in WVU Graduate Theses, Dissertations, and Problem Reports collection by an authorized administrator of The Research Repository @ WVU. For more information, please contact researchrepository@mail.wvu.edu. 


\title{
Impedance Effects of Metallic Ground Plane on the CTHA Antenna
}

\author{
Tao Guan
}

Thesis submitted to the faculty of the College of Engineering and Mineral Resources at West Virginia University in partial fulfillment of the requirements for the degree of

\author{
Master of Science \\ in \\ Mechanical Engineering \\ James E. Smith, Ph.D., Chair \\ Roy S. Nutter, Ph.D. \\ Gregory J. Thompson, Ph.D. \\ October 26, 1998 \\ Morgantown, West Virginia
}

Keywords: CTHA, Impedance, Experimental, Numerical, Antenna, Ground Plane 


\title{
Impedance Effects of Metallic Ground Plane on the CTHA Antenna
}

\author{
Tao Guan
}

\begin{abstract}
As with the fast-paced development of the communication industry, antennas have become an indispensable part of daily life. However, everyone may have experienced the malfunction of a communication system or radio due to a nearby human body or building or automobile. Then comes the question of how adjacent objects influence the antenna. This thesis has concentrated on the study of these effects on the impedance of the CTHA (contrawound toroidal helical antenna).

The results of this study indicate the CTHA's input impedance changes as a damped sinusoid function of the distance between it and the metallic ground plane. For equivalent distances between the antenna and the metallic ground plane, the CTHA's impedance has been shown to be less affected than the impedance of a vertical dipole, a horizontal dipole, or a horizontal loop antenna. This has been shown by both simulation and experiment and is demonstrated by the plot of the magnitude of the relative impedance change. It indicates that the CTHA will have a smaller increase of the SWR than the other antennas when foreign objects are in the vicinity of the antenna.
\end{abstract}

This study has also confirmed that NEC (numerical electromagnetic code) is an effective numerical tool for modeling dipoles and loop antennas. The inability for NEC to accurately model the CTHA seems to be caused by the inability to include effects of the core material with dielectric constant other than 1 of the CTHA. Another numerical method, XFDTD (X-window finite difference time domain method), has shown better accuracy in calculating the CTHA's impedance than NEC when comparing with the experimental results. 


\section{Acknowledgments}

The author would like to thank Dr. Roy Nutter, Dr. Gregory Thompson and Dr. James Smith for agreeing to be on the committee. Their assistance and critical comments to the research work and thesis have been very valuable to the accomplishment of this thesis. The author is particularly indebted for support and encouragement to Dr. Smith. Without his aid, the research would not be able to be completed. Besides, the author also wants to express the appreciation to the help from Robert Craven, Franz Andy Pertl, Joanna Davis-Swing, and Khaled Elsherbini. Their suggestions and technical supports have been very helpful throughout the whole research and the completion of the thesis. It is the author's honor to work with the team-Center for Industrial Research Applications (CIRA). It provides a wonderful environment for the author to obtain knowledge and skills on one hand, and employ her knowledge and skills to implement technical research on the other hand. It is a precious experience.

Finally, the author wants to thank her parents Zhihong Guan and Qihui Xu, and her husband Xiaotao Mei, who have given her so much love and encouragement. Each of her achievements should be attributed to them. 


\section{Table of Contents}

Abstract

$i i$

Acknowledgments

iii

Table of Contents iv

List of Figures vii

List of Tables ix

Nomenclature $x$

Chapter 1 Introduction 1

1. Background 1

2. Contrawound Toroidal Helical Antenna (CTHA)___ 2

3. Objective ___ 3

4. Methodology _______ 4

5. Literature Review_____ 4

1) Antenna Input Impedance___ 4

2) Related Research __ 7

Chapter 2 Modeling with NEC for Impedance Study___ 12

1. Introduction of NEC ______________ 12

2. Modeling for Metal Plate Study ___ 13

3. Numerical Result ___ _ 17

1) Vertical Dipole over 3ft x 3ft Metal Plate __ 17

2) Horizontal Dipole over $3 \mathrm{ft} \times 3 \mathrm{ft}$ Metal Plate __ 21

3) Horizontal Loop over $3 \mathrm{ft} \times 3 \mathrm{ft}$ Metal Plate __ 22

4) Horizontal CTHA over 3ft x 3ft Metal Plate _ 22

4. Summary ___ 23

Chapter 3 Measurement of Antennas' Input Impedance __ 25

1. Introduction of Equipment ___ 25 
1) Anechoic Chamber__ 25

2) HP 8753D Network Analyzer __ 26

3) Setup of the $3 \mathrm{ft} \times 3 \mathrm{ft}$ Metal Plate Study ___ 27

4) Measurement Calibration __ 28

2. Experimental Results ___ 30

3. Error Analysis of Impedance Measurement ___ 32

Chapter 4 Comparison of NEC and Experimental Results __ 33

1. Introduction __ 33

2. Vertical Dipole ___ 33

3. Horizontal Dipole ___ 35

4. Horizontal Loop ___ 36

5. Horizontal CTHA___ 38

6. Summary ___ 42

Chapter 5 Applying XFDTD on Impedance Study ___ 44

1. Introduction ___ 44

2. Evaluation of XFDTD ___ 44

3. Applying XFDTD on the Metal Plate Study of the CTHA _ 46

4. Summary ___ 51

Chapter 6 Comparison of Antennas __ 52

1. Introduction __ 52

2. Magnitude of the Relative Impedance Change ___ 52

3. Effect of the Relative Impedance Change on SWR___ 53

4. Change of the Resonance Frequency __ 56

5. Exploration of the Reasons for CTHA's Relative Impedance Invariance____ 57

1) Effect of Wire Coupling__ 57

2) Effect of Near Field Energy Distribution __ 60

6. Summary ___ 64

Chapter 7 Conclusions and Recommendations __ 66

1. Conclusions ___ 66

2. Recommendations____ 67

References _— 68 
Appendix B Plain Surface Generator 85

Appendix C Properties of RANTEC FerroSorb 89

Appendix D Specifications of HP8753D Network Analyzer 90 Approval of Examining Committee 91 


\section{List of Figures}

Figure 1 Geometry of the CTHA 3

Figure 2 Equivalent Circuit to a Transmitting Antenna [8]

Figure 3 Input Impedance of a Horizontal Wire antenna above the Earth $(\varepsilon 1 r=10.0$,

$$
\left.\sigma l=1.0 \times 10^{-3} \mathrm{~S} / \mathrm{m}\right)[9]
$$

Figure 4 Vertical Electric Dipole Impedance as a Function of Height above a Homogeneous Lossy Half-Space [8,10] 10

Figure 5 Vertical Magnetic Dipole (or small Horizontal Loop) Impedance Change as a Function of Height above a Homogeneous Lossy Half-Space [8,10]

Figure 6 Antennas above a 3ft x 3ft Metal Plate 14

Figure 7 Closed Patch Surfaces 18

Figure 8 Relative Impedance Change of the Vertical Dipole over the 3'x 3'Metal Plate Modeled with Different Surface Elements 20

Figure 9 Vertical Dipole Numerical Results for Different Plate Discretization 21

Figure 10 Input Impedance of Horizontal Dipole in a Function of Relative Height 21

Figure 11 3'x3' Metal Plate under the CTHA inside the CIRA Anechoic Chamber 26

Figure 12 Picture of the Testing Site 28

Figure 13 Diagram of the Setup of Impedance Measurement 28

Figure 14 Measured Free Space Impedance of a Half-Wave Dipole 30

Figure 15 Relative Impedance Change of the Vertical Dipole 34

Figure 16 Resistance and Reactance of the Vertical Dipole as a Function of the Relative Height 35

Figure 17 Relative Impedance Change of the Horizontal Dipole 36

Figure 18 Resistance and Reactance of the Horizontal Dipole as a Function of the Relative Height 36

Figure 19 Relative Impedance Change of the Horizontal Loop 37

Figure 20 Resistance and Reactance of the Horizontal Loop as a Function of the Relative Height 37 
Figure 21 Relative Impedance Change of the Horizontal CTHA

Figure 22 Resistance and Reactance of the Horizontal CTHA as a Function of the Relative Height 42

Figure 23 Relative Impedance Change of the Vertical Dipole over a 3'x3'Metal Plate 46

Figure 24 Relative Impedance Change of the CTHA over a 4"x4" Metal Plate

Figure 25 Magnitude of the Relative Impedance Change of the CTHA over a 4"x4" Metal Plate 51

Figure 26 Magnitude of the relative Impedance Change Resulted from the Experimental Results 53

Figure 27 Resonance Frequency Reference to the Free Space Resonance Frequency. 56

Figure 28 Relative Impedance Change of the Single Helix and the CTHA Resulted from NEC 59

Figure 29 Magnitude of the relative Impedance Change of the Single Helix and the CTHA Resulted from NEC. 60

Figure 30 Near-Field Intensity of Horizontal Dipole 61

Figure 31 Near-Field Intensity of CTHA 62

Figure 32 Comparison of Electric Flux Density 63

Figure 33 Comparison of Magnetic Flux Density 64

Figure 34 Plain_form 85

Figure 35 Form of Keypoint Data File Generator 86

Figure 36 Form of Grid Data File Generator 87 


\section{List of Tables}

Table 1 Nomenclatures in the Thevenin Circuit Equivalent

Table 2 Specifications of Antennas 15

Table 3 Distances from the Antennas to the Metal Plate Used for Impedance Study (mm) 16

Table 4 Free Space Frequencies and Impedance of the CTHA in NEC Analysis 23

Table 5 Free Space Frequencies and Impedance 31

Table 6 Percentage Difference of Numerical Result to Experimental Result 31

Table 7 Free Space Resonance Frequency and Impedance of the Vertical Dipole 45

Table 8 Percentage Difference of the Numerical Results to the Experimental Results 45

Table 9 Free Space Resonance Frequencies and Impedance of the CTHA 47

Table 10 Percentage Difference of the Numerical Results to the Experimental Results 47 


\title{
Nomenclature
}

\author{
B Magnetic flux density \\ D Electric flux density \\ E Electric field intensity \\ Ei ith data of the experimental results set \\ $\mathrm{H} \quad$ Magnetic field intensity \\ Ig Current in the loop \\ $\mathrm{m} \quad$ Magnitude of the relative impedance change \\ n Total number of data in a data set \\ $\mathrm{Ni}$ ith data of the numerical results set \\ $\operatorname{Pr} \quad$ Power delivered to the antenna for radiation \\ r Radius of wire-grid segment \\ $\mathrm{R} \quad$ Resistance of the antenna \\ R0 Free space resistance of the antenna \\ $\mathrm{Ra} \quad$ Load resistance at terminated end of transmission line \\ RA Antenna's resistance at terminals $a-b$ \\ Rc Characteristic resistance of transmission line \\ $\operatorname{Rg} \quad$ Resistance of the generator and transmission line impedance \\ $\mathrm{RL} \quad$ Loss resistance of the antenna \\ $\mathrm{Rr} \quad$ Radiation resistance of the antenna \\ Vg Peak generator voltage \\ X Reactance of the antenna \\ X0 Free space reactance of the antenna \\ $\mathrm{Xa} \quad$ Load reactance at terminated end of transmission line \\ XA Antenna's reactance at terminals a-b \\ Xc Characteristic reactance of transmission line \\ $\mathrm{Xg} \quad$ Reactance of the generator and transmission line impedance \\ ZA Antenna's impedance at terminals $a-b$ \\ Zc Complex characteristic impedance of transmission line \\ $\mathrm{Zg} \quad$ Internal impedance of the generator \\ $\Delta \quad$ Separation of wires in the mesh
}


E0 Permittivity of free space $(\approx 8.854 \mathrm{E}-12 \mathrm{~F} / \mathrm{m})$

$\lambda \quad$ Wavelength

$\lambda 0 \quad$ Free space wavelength

$\mu 0 \quad$ Permeability of free space $(\approx 1.257 \mathrm{E}-6 \mathrm{H} / \mathrm{m})$

$\rho \quad$ Reflection coefficient

$\sigma \mathrm{E} \quad$ Standard deviation of the experimental results set

$\sigma \mathrm{N}$ Standard deviation of the numerical results set 


\section{Chapter 1 Introduction}

\section{Background}

Over the last 50 years, communication technology has experienced a breathtaking revolution; the antenna has played an indispensable role in that revolution. The antenna serves a communication system the same way that eyes and ears serve a human. More than that, the antenna has been one of the most critical components of some new technologies, such as GPS

(Global Positioning System), keyless entry, etc. The need for exploring better antenna designs for all kinds of purposes is well recognized.

As defined in IEEE Transactions on Antennas and Propagation [1], an antenna is "a means for radiating or receiving radio waves." Consequently the amount of energy generated from a source that can be radiated or received through an antenna is the primary consideration of antenna design.

In general, an antenna is a special component of an electrical circuit, in which the antenna is connected to a source with a transmission line. Under ideal conditions, the energy generated by the source should be totally transferred to the antenna through a transmission line, and then radiated to space. But in a practical system, there are conduction-dielectric losses due to the lossy nature of the transmission line and the antenna, as well as reflection (mismatch) losses at the interface between the line and the antenna. The maximum radiation occurs when the system is under conjugate matching.

System matching is the match between the impedance of transmission line, the tuning circuit (matching network), and the impedance of antenna. When the impedance of the transmission line 
and the tuning circuit is fixed, the system matching depends on the stability of the impedance of the antenna. The impedance of an antenna is made up of self-impedance and mutual impedance [2]. Self-impedance is the impedance that would be measured at the terminals of the antenna if it were in free space with no other antennas or reflecting objects in the vicinity. Mutual impedance accounts for the coupling between the antenna and any other source [3]. Usually, the system matching is designed by taking into account the antenna's self-impedance. In practice, all kinds of objects can exist in the vicinity of an antenna, such as human bodies, automobiles, buildings, etc. These objects could affect the antenna's mutual impedance significantly, which may result in a very poor performance of the antenna.

This research has focused on studying the impedance effect of metallic ground plane on the Contrawound Toroidal Helical Antenna (CTHA). Both experimental and numerical techniques have been used to study the CTHA.

\section{Contrawound Toroidal Helical Antenna (CTHA)}

"The Toroidal Helical Antenna (THA) is a new antenna design that has promising performance as it has a small profile compared to the commercial standard dipole antennas of the same resonance frequency" [6]. As an important class of THA, the Contrawound Toroidal Helical Antenna (CTHA), which has two helices with uniform pitches and opposite senses and one feeding port as shown in Figure 1, not only has the advantage of being physically small, but also has the advantages of being omnidirectional in multiple axis (isotropic radiator), circularly polarized and scaleable design [7]. With these advantages, the CTHA has promising potentials for wide applications. 
This research studies the impedance variance of the CTHA in the vicinity of a metallic ground plane. Both numerical and experimental results are obtained, compared, and contrasted against the same results for a vertical dipole, a horizontal dipole, and a horizontal loop.

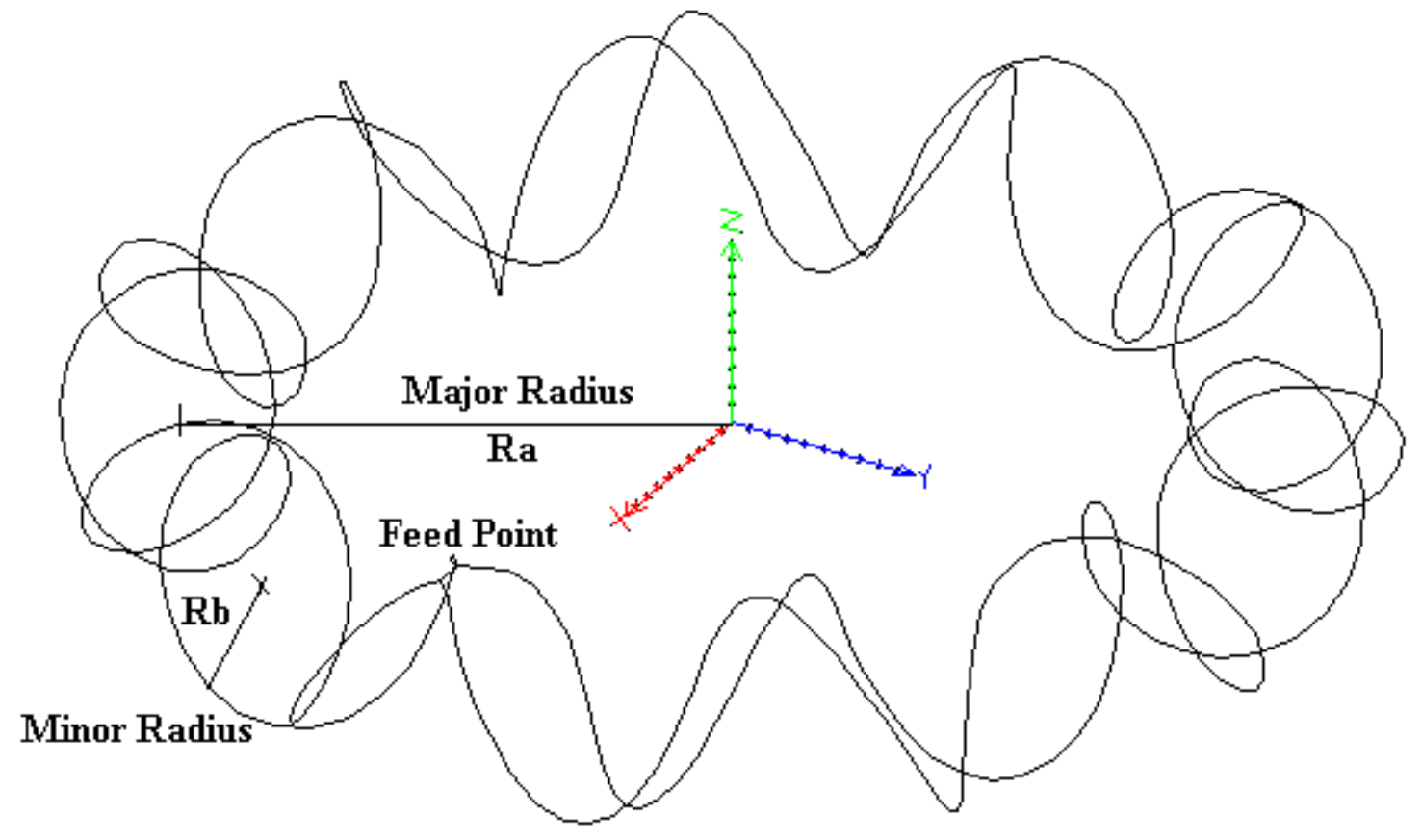

Figure 1 Geometry of the CTHA

\section{Objective}

The primary interest of this thesis is to study the effects of varying the distances between the CTHA and the metallic ground plane on the impedance of the CTHA. The impedance effect of the metallic ground plane on the CTHA is to be compared with that on the other antennas. The reasons for its relative impedance invariance are to be explored. For conducting this impedance study, both numerical and experimental approaches have been employed. The effectiveness of the numerical methods for studying the CTHA's impedance is to be evaluated. 


\section{Methodology}

Both numerical and experimental approaches have been employed in this impedance study. The numerical methods involved include NEC (Numerical Electromagnetics Code) [11] and XFDTD (X-Window Finite Difference Time Domain) [20]. The measurement of the antenna's impedance was conducted in an anechoic chamber with the HP8753D network analyzer.

\section{Literature Review}

\section{1) Antenna Input Impedance}

As mentioned above, an antenna is a special kind of electrical circuit. Figure 2 shows the Thevenin circuit equivalent of an antenna. The nomenclatures involved in this circuit equivalent are listed in Table 1. The input impedance of an antenna is defined as the impedance presented by an antenna at its terminals or the ratio of the voltage to current at a pair of terminals [8] and is given as

$$
Z_{A}=R_{A}+j X_{A}
$$

In general, the resistive part of Eq.1-1 consists of two components $R_{r}$ and $R_{L}$ as indicated in Eq.1-2. The generator and transmission line has internal impedance as expressed in Eq.1-3.

$$
\begin{aligned}
& R_{A}=R_{r}+R_{L} . \\
& Z_{g}=R_{g}+j X_{g} .
\end{aligned}
$$


Table 1 Nomenclatures in the Thevenin Circuit Equivalent

\begin{tabular}{|c|l|}
\hline Term & \\
\hline $\mathrm{Ig}$ & the current in the loop \\
\hline $\mathrm{P}_{\mathrm{r}}$ & the power delivered to the antenna for radiation \\
\hline $\mathrm{R}_{\mathrm{A}}$ & the antenna's resistance at terminals a-b \\
\hline $\mathrm{R}_{\mathrm{g}}$ & the resistance of the generator and transmission line impedance \\
\hline $\mathrm{R}_{\mathrm{L}}$ & the loss resistance of the antenna \\
\hline $\mathrm{R}_{\mathrm{r}}$ & the radiation resistance of the antenna \\
\hline $\mathrm{V}_{\mathrm{g}}$ & the peak generator voltage \\
\hline $\mathrm{X}_{\mathrm{A}}$ & the antenna's reactance at terminals a-b \\
\hline $\mathrm{X}_{\mathrm{g}}$ & the reactance of the generator and transmission line impedance \\
\hline $\mathrm{Z}_{\mathrm{A}}$ & the antenna's impedance at terminals a-b \\
\hline
\end{tabular}




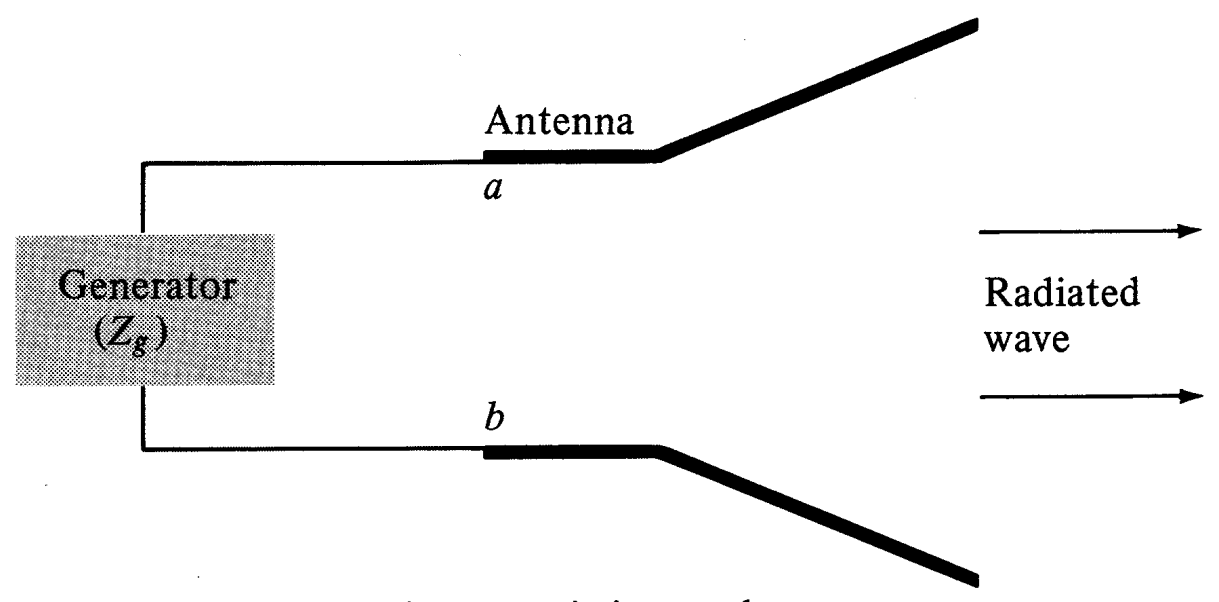

(a) Antenna in transmitting mode

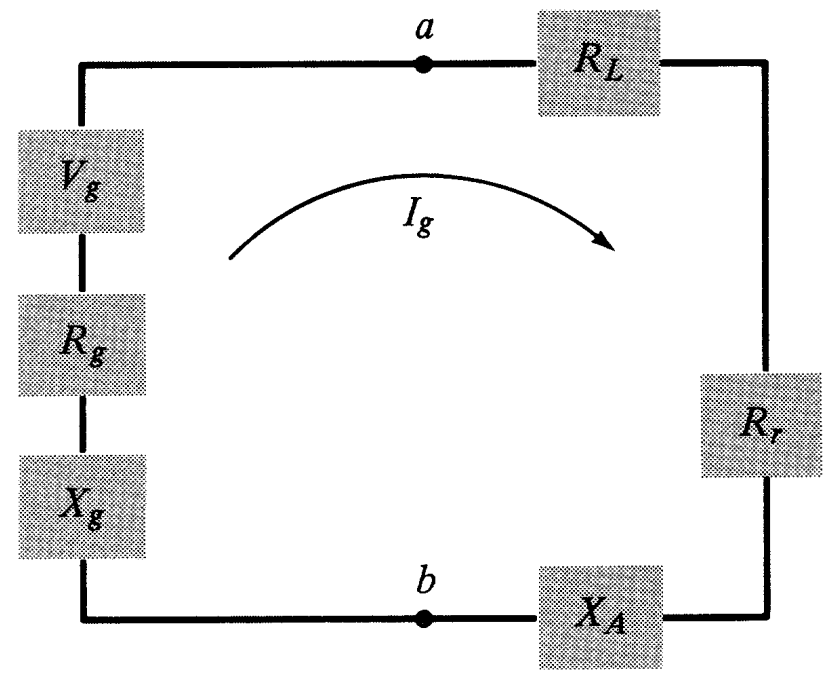

(b) Thévenin equivalent

Figure 2 Equivalent Circuit to a Transmitting Antenna [8]

According to the Thevenin equivalent circuit and circuit theory, the current in the system can be expressed as Eq.1-4 and the power delivered to the antenna for radiation can be expressed as Eq.1-5.

$$
I_{g}=\frac{V_{g}}{Z_{A}+Z_{g}}=\frac{V_{g}}{\left(R_{r}+R_{L}+R_{g}\right)+j\left(X_{A}+X_{g}\right)}
$$




$$
P_{r}=\frac{1}{2}\left|I_{g}\right|^{2} R_{r}=\frac{\left|V_{g}\right|^{2}}{2}\left[\frac{R_{r}}{\left(R_{r}+R_{L}+R_{g}\right)^{2}+\left(X_{A}+X_{g}\right)^{2}}\right]
$$

From Eq.1-5, it can be clearly found that the maximum power delivered to the antenna occurs when conjugate matching is reached by satisfying:

$$
R_{r}+R_{L}=R_{g},
$$

and

$$
X_{A}=-X_{g} .
$$

When there is a metallic ground plane in the vicinity of the antenna, the antenna's impedance $Z_{A}$ will change. That is to say the value of $R_{A}$, which is equal to $R r+R_{L}$, and $X_{A}$ may no longer equal to $R_{g}$ and $-X_{g}$, respectively. Consequently, the conjugate matching of the system will not be satisfied. This will lead to the decrease of the power delivered to the antenna. By studying how the metallic ground plane affects the impedance of the CTHA, it is expected to help to find compensations for maintaining the system matching in the case when there are objects in the vicinity of the CTHA.

\section{2) Related Research}

Several studies on the impedance effects of adjacent objects on antennas have been conducted. For example, R. C. Babu's "Impedance Calculations for Horizontal Wire Antennas above a Lossy Earth" [9] and Constantain A. Balanis' study of ground effects on an infinitesimal vertical dipole, and a small horizontal loop [8]. But this study is the first effort to study the impedance effects of the metallic ground plane on the CTHA. The conclusions of Babu's study and Balanis' study have provided valuable references for validating the approaches used in this 
research for studying CTHA's impedance, and also provided guidance for studying the impedance effects of the metallic ground plane on the CTHA.

In the Babu's study [9], the input impedance of a thin cylindrical wire antenna kept horizontally above a lossy earth is calculated by solving the electric field integral equation for the antenna current using the method of moments (MoM). Coupled horizontal wire antennas kept at the same height from the air-earth interface are analyzed by solving the corresponding zero and first phase sequence current integral equations. The input impedance as a function of antenna height (in terms of free space wavelength) from the air-earth interface is shown in Figure 3. "For the finite conductivity of the earth, the impedance of a horizontal antenna is maximum when it is kept closest to the interface; it reaches a minimum as the separation from the interface is increased, and then increases and finally reaches the free space value at large distances from the boundary" [9].

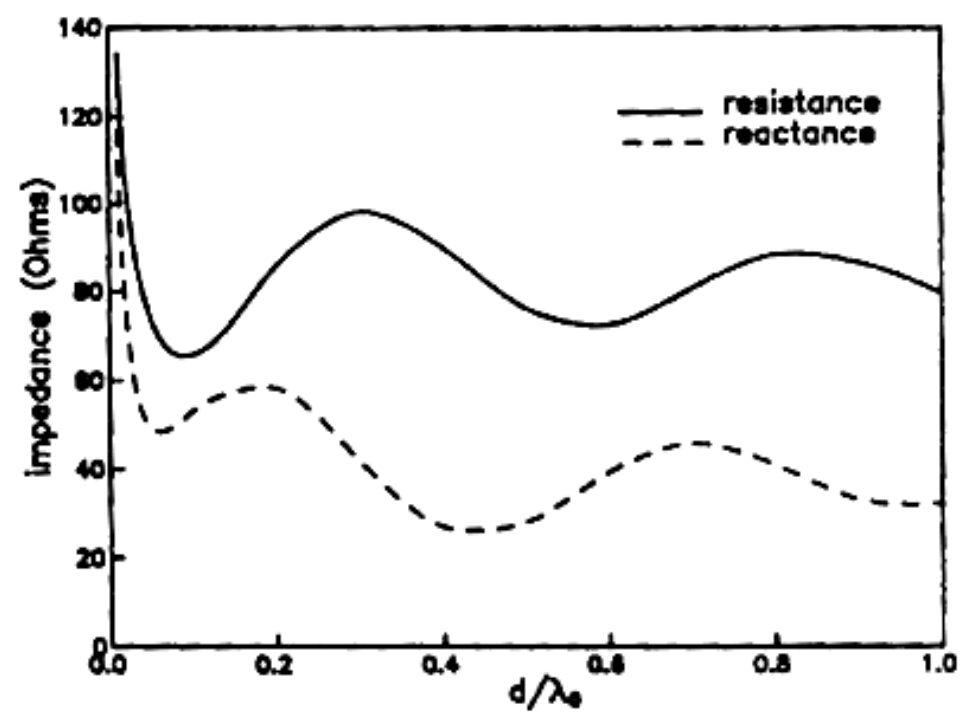

Figure 3 Input Impedance of a Horizontal Wire antenna above the Earth $(\varepsilon 1 r=10.0$, $\left.\sigma 1=1.0 \times 10^{-3} \mathrm{~S} / \mathrm{m}\right)[9]$ 
Babu's study has disclosed that the antenna's resistance and reactance change as a function of the distance between the antenna and the ground plane relative to the free space wavelength. In this study, the function of the resistance and reactance of the CTHA has been studied.

In the Balanis' study [8], the impedance change of an infinitesimal vertical dipole and a small loop placed a height above a homogeneous lossy half-space has been compared to the impedance in free space radiation. The relative resistance change is expressed as Eq.1-8 and the relative reactance change is expressed as Eq.1-9.

$$
\begin{aligned}
& \Delta R / R_{0}=\left(R-R_{0}\right) / R_{0} \\
& \Delta X / R_{0}=\left(X-X_{0}\right) / R_{0}
\end{aligned}
$$

Graphical illustration of the relative impedance change of the infinitesimal vertical dipole is shown in Figure 4, and that of the small loop is shown in Figure 5. Balanis' results indicate that the antennas' relative impedance change decreases along spiral curves when the distance between the antenna and the lossy ground increases. When the distance tends to be infinity, the relative impedance change tends to be zero. This approach provides a combined consideration on both the resistance change and the reactance change. In addition, it references the impedance change to the free space impedance, which makes the impedance effects comparable among different antennas at different frequencies. In this impedance study, this analysis method has been extensively used on studying the impedance effects of the metallic ground plane on the CTHA. 


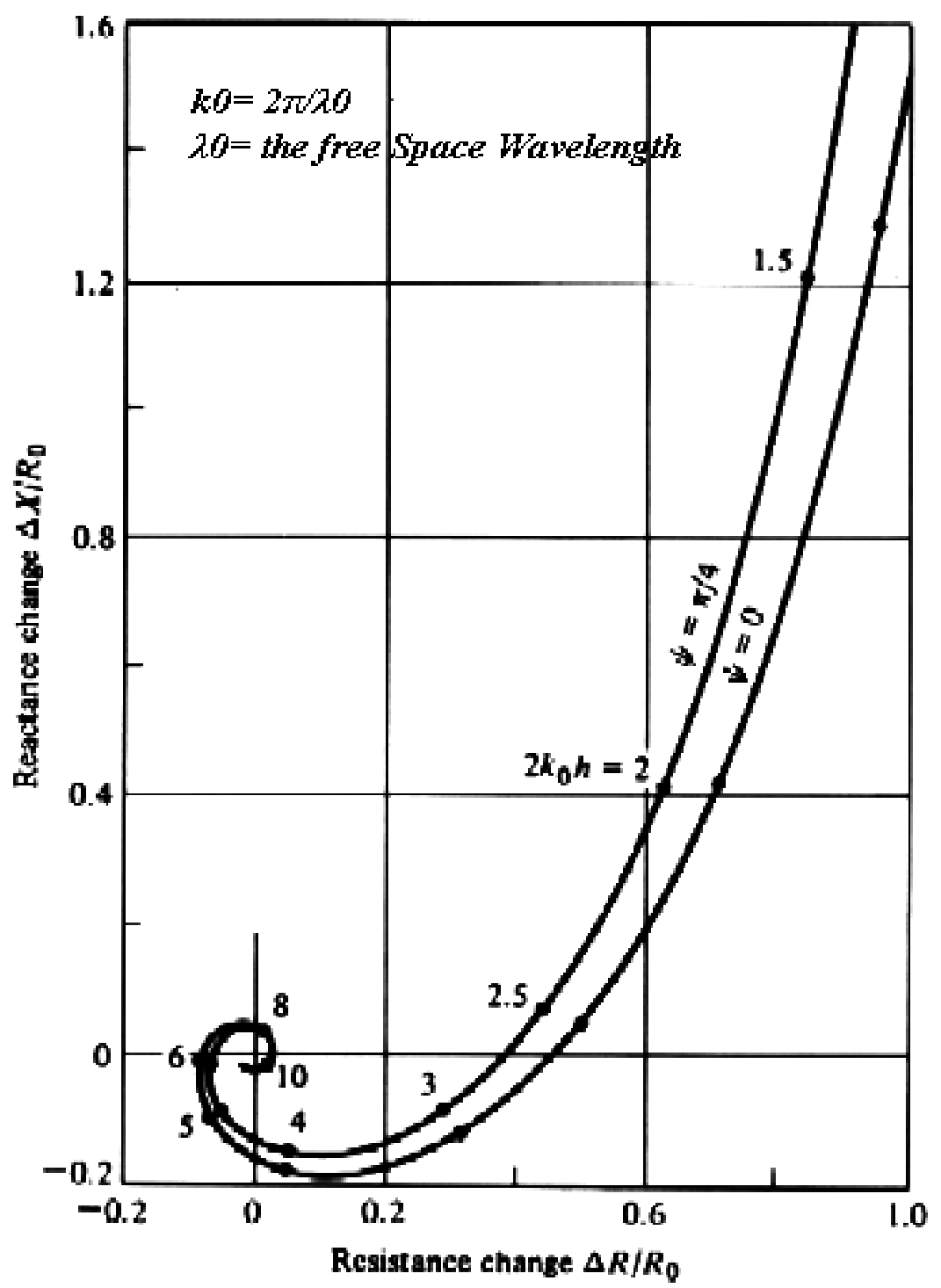

Figure 4 Vertical Electric Dipole Impedance as a Function of Height above a Homogeneous Lossy Half-Space $[8,10]$ 


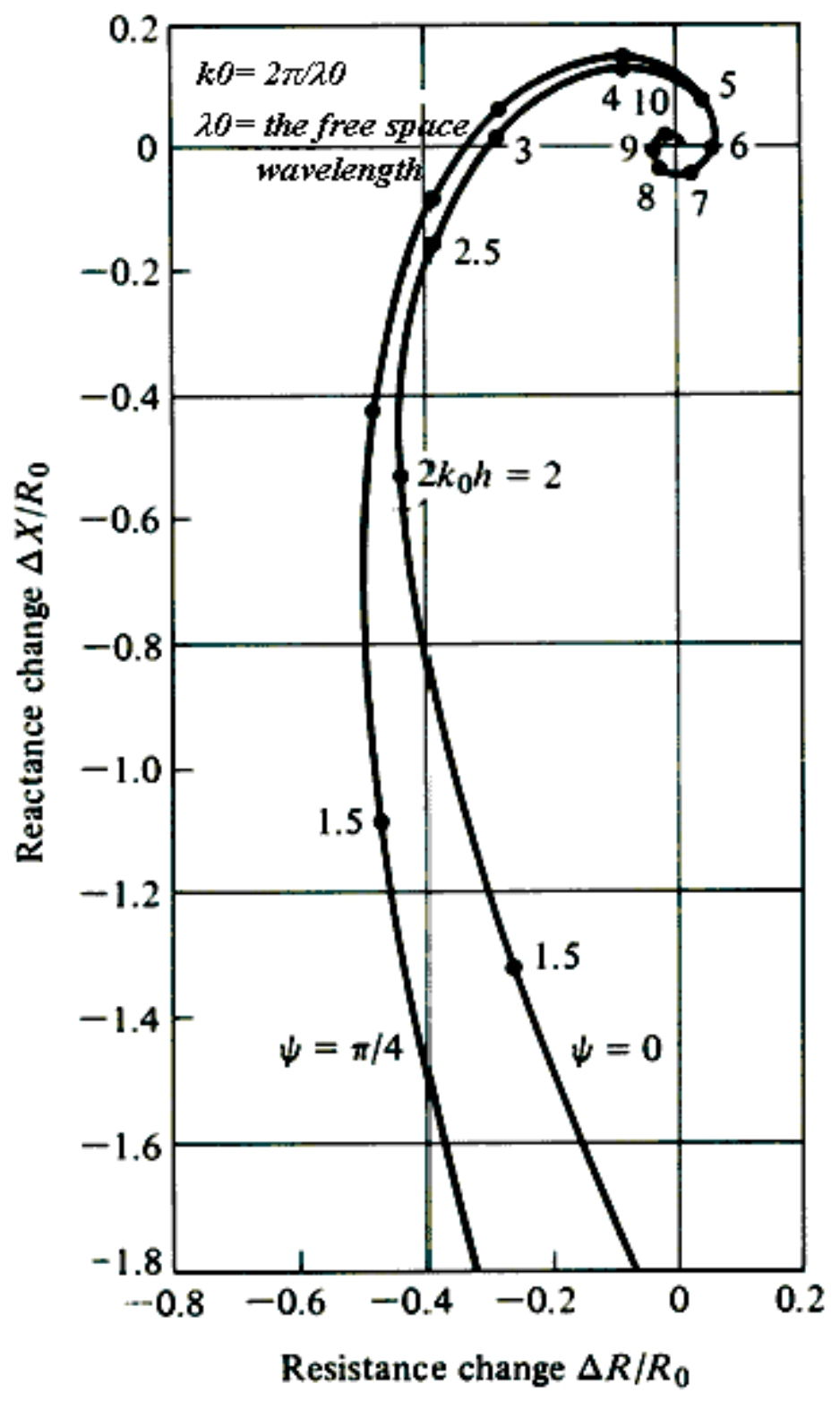

Figure 5 Vertical Magnetic Dipole (or small Horizontal Loop) Impedance Change as a Function of Height above a Homogeneous Lossy Half-Space [8,10] 


\section{Chapter 2 Modeling with NEC for Impedance Study}

For studying the impedance effects of a metallic ground plane on the CTHA, a $3 \mathrm{ftx} 3 \mathrm{ft}$ metal plate was modeled at a distance beneath a horizontal CTHA. In order to validate the effectiveness of NEC in impedance calculation, the $3 \mathrm{ftx} 3 \mathrm{ft}$ metal plate has also been modeled under a vertical dipole, a horizontal dipole and a horizontal loop.

\section{Introduction of NEC}

The Numerical Electromagnetics Code (NEC) is "a computer program for analyzing the electromagnetic response of antennas and scatterers. The code is based on the numerical solution of integral equations by the method of moments; it combines an electric-field integral equation for modeling thin wires with a magnetic-field integral equation for closed, perfect conducting surfaces" [11].

NEC provides two options for modeling a conducting surface, namely wire-grid and surface patch. Modeling conducting surfaces with the wire-grid feature applies the equivalent of a solid conducting surface to a grid having sufficiently small mesh size. According to the description in [11], the segment length of wire should be less than $0.1 \lambda$ ( $\lambda$ is the wavelength), and the circumference of wire segment should be less than the separation of wires. This can be used to model thin plates, open shells, and finitely conducting surfaces. Modeling conducting surfaces with the surface patch option uses the magnetic field integral equation. It is restricted to closed surfaces with a non-vanishing enclosed volume. However, results from modeling a thin reflection surface with surface patch turn out to be quite close to the experimental results, as shown in Chapter 4. For evaluating the accuracy of NEC results, wire-grid, closed patch surfaces 
and thin plate composed of surface patch have been used to model the $3 \mathrm{ft} x 3 \mathrm{ft}$ metal plate separately.

\section{Modeling for Metal Plate Study}

The metal plate study is set up as shown in Figure 6. A horizontal CTHA (details as shown in Figure 1) is placed with its major radius parallel to the plate; a vertical dipole is placed with the wire perpendicular to the plate; a horizontal dipole is placed with the wire parallel to the plate and also parallel to two sides of the plate; and a horizontal loop is placed with its radius parallel to the plate. All antennas have been centered to the center of metal plate.

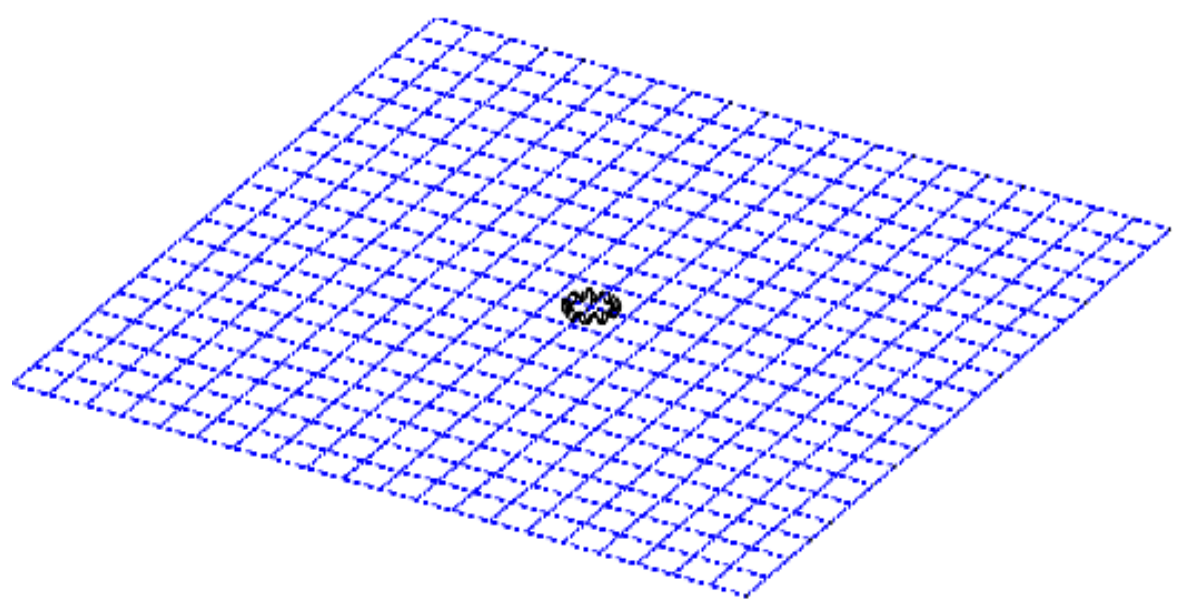

(a) Horizontal CTHA 


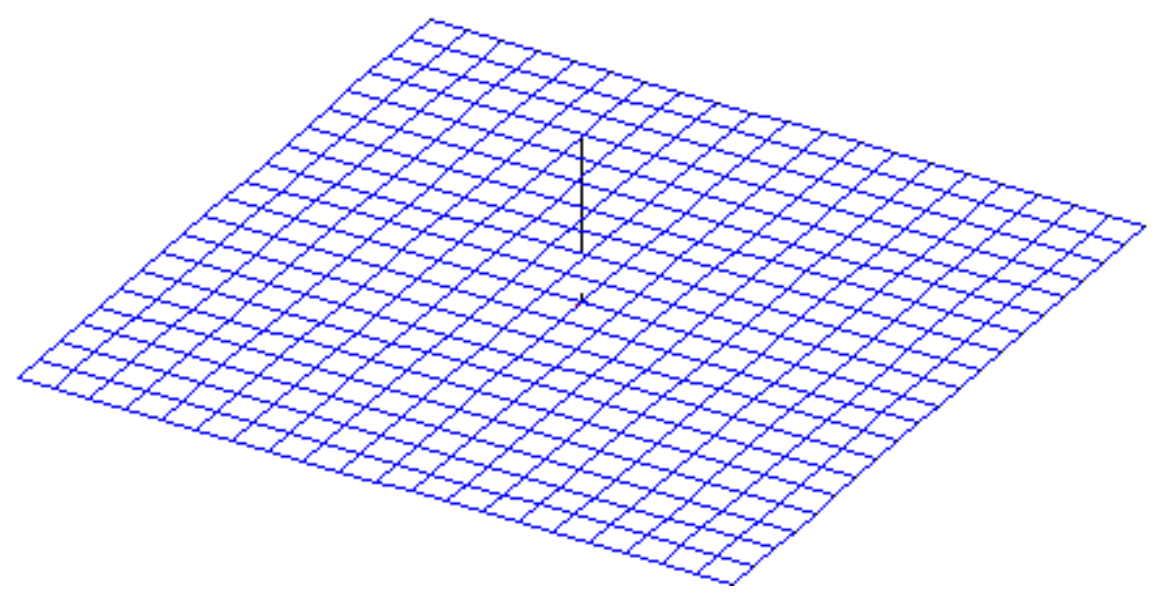

(b) Vertical Dipole

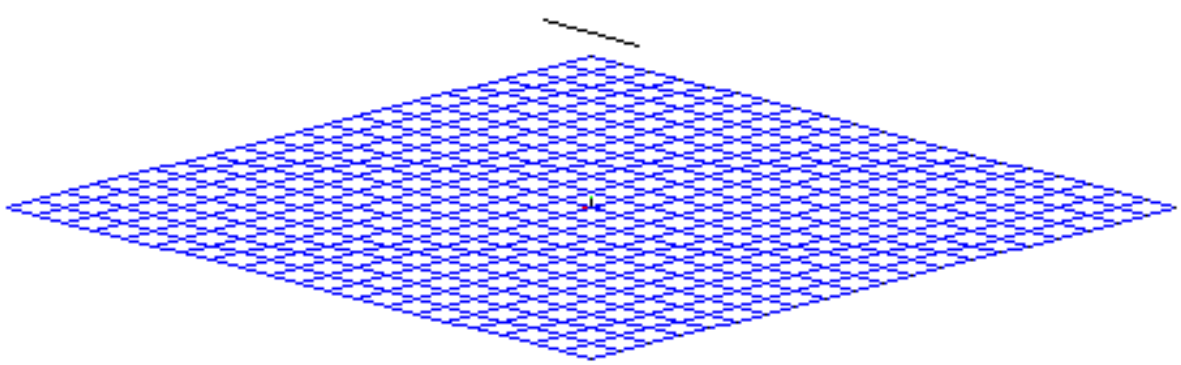

(c) Horizontal Dipole

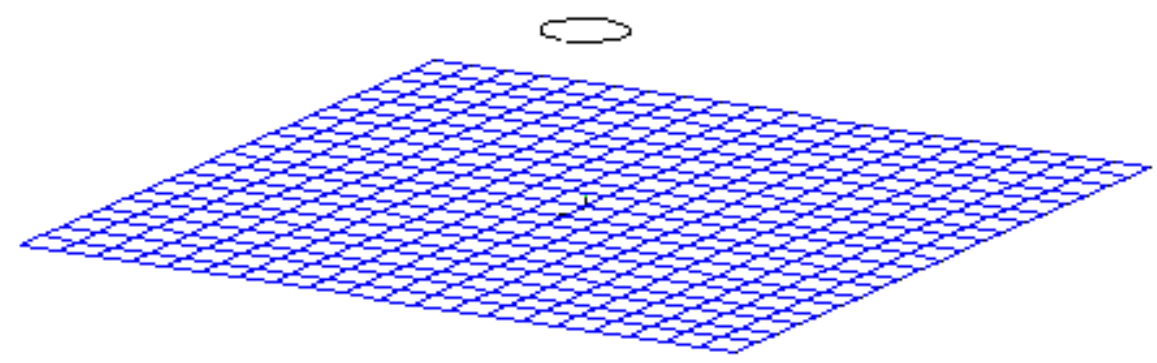

(d) Horizontal Loop

Figure 6 Antennas above a 3ft x 3ft Metal Plate

The NEC cards for modeling the antennas over a $3 \mathrm{ft} \times 3 \mathrm{ft}$ metal plate have been shown in Appendix A. The geometry specifications of the antennas are listed in Table 2. 
Table 2 Specifications of Antennas

\begin{tabular}{|l|c|c|c|}
\hline & CTHA & Dipole & Loop \\
\hline Major Radius & $0.02667 \mathrm{~m}$ & - & $0.04978 \mathrm{~m}$ \\
Minor Radius & $4.7 \mathrm{~mm}$ & - & - \\
Wire Radius & $0.36 \mathrm{~mm}$ & $1.016 \mathrm{~mm}$ & $0.5206 \mathrm{~mm}$ \\
Wire Length & $0.376 \mathrm{~m}+0.378 \mathrm{~m}$ & $0.1435 \mathrm{~m}$ & $0.313 \mathrm{~m}$ \\
Number of Turns & 10 & - & 1 \\
Crossing & Top & - & - \\
Feed Type & 6 & - & - \\
\hline
\end{tabular}

The plain surface generator for generating geometry cards for modeling surfaces with a wiregrid and a surface patch is programmed with Visual Basic. A brief introduction of the function of the generator is in Appendix B. To move the antennas along the $\mathrm{Z}$-axis and rotate the vertical loop to horizontal by $90^{\circ}$ around the $\mathrm{X}$-axis, a "GM"—coordinate transformation card, is used. The distance between the antenna and the plate is measured from the bottom of the antenna to the plate. For each antenna, 20 different distances are calculated. The distances from the bottom of the antenna to the metal plate and the corresponding distances from the feed point of the antenna to the metal plate are listed in Table 3. For most antennas, the testing frequency is around $1 \mathrm{GHz}$, this corresponds to a free space wavelength of $300 \mathrm{~mm}$. Variable steps have been chosen for different distance ranges due to that the closer the antenna to the plate, the more its impedance changes. 
Table 3 Distances from the Antennas to the Metal Plate Used for Impedance Study (mm)

\begin{tabular}{|c|c|c|c|c|}
\hline \multirow{2}{*}{$\begin{array}{l}\text { Bottom to } \\
\text { Plate }\end{array}$} & \multicolumn{4}{|c|}{ Feed Point to Plate } \\
\hline & Hori. CTHA & Vert. Dipole & Hori. Dipole & Hori. Loop \\
\hline 1 & 11 & 73 & 2 & 1.5 \\
\hline 5 & 15 & 77 & 6 & 5.5 \\
\hline 10 & 20 & 82 & 11 & 10.5 \\
\hline 15 & 25 & 87 & 16 & 15.5 \\
\hline 20 & 30 & 92 & 21 & 20.5 \\
\hline 25 & 35 & 97 & 26 & 25.5 \\
\hline 30 & 40 & 102 & 31 & 30.5 \\
\hline 35 & 45 & 107 & 36 & 35.5 \\
\hline 40 & 50 & 112 & 41 & 40.5 \\
\hline 50 & 60 & 122 & 51 & 50.5 \\
\hline 60 & 70 & 132 & 61 & 60.5 \\
\hline 70 & 80 & 142 & 71 & 70.5 \\
\hline 80 & 90 & 152 & 81 & 80.5 \\
\hline 100 & 110 & 172 & 101 & 100.5 \\
\hline 130 & 140 & 202 & 131 & 130.5 \\
\hline 160 & 170 & 232 & 161 & 160.5 \\
\hline 190 & 200 & 262 & 191 & 190.5 \\
\hline 220 & 230 & 292 & 221 & 220.5 \\
\hline 250 & 260 & 322 & 251 & 250.5 \\
\hline 300 & 310 & 372 & 301 & 300.5 \\
\hline
\end{tabular}




\section{Numerical Result}

\section{1) Vertical Dipole over 3ft x 3ft Metal Plate}

For a vertical dipole, three kinds of surfaces have been modeled, wire-grid, thin patch surface and closed patch surfaces.

I. The wire grid surface is constructed as a square mesh of segments. For accuracy, a mesh size of $30.5 \mathrm{~mm}$ is chosen, which is about $0.1 \lambda$. The wire radius of the wire-grid is $4.775 \mathrm{~mm}$, which agrees with the rule for wire modeling [11]:

$$
2 \pi r=\Delta
$$

A total number of 1,860 wire segments are used to model the $3 \mathrm{ft} \times 3 \mathrm{ft}$ metal plate.

II. The thin patch surface is constructed with the square patch element. A total number of 400 (20x20) patch elements are used to model the metal plate. The individual area of each patch is $2.09 \mathrm{E}-3 \mathrm{~m}^{2}$, which is less than $3.6 \mathrm{E}-3 \mathrm{~m}^{2}\left(0.04 \lambda^{2}\right)$ required for accuracy [11]. The normal vector of each patch element points upward.

III. Closed patch surfaces are made up of six thin patch surfaces as illustrated in Figure 7. All patch elements involved have the same size and shape. The normal vector of each element is directed outward. A total number of 880 elements are used. 


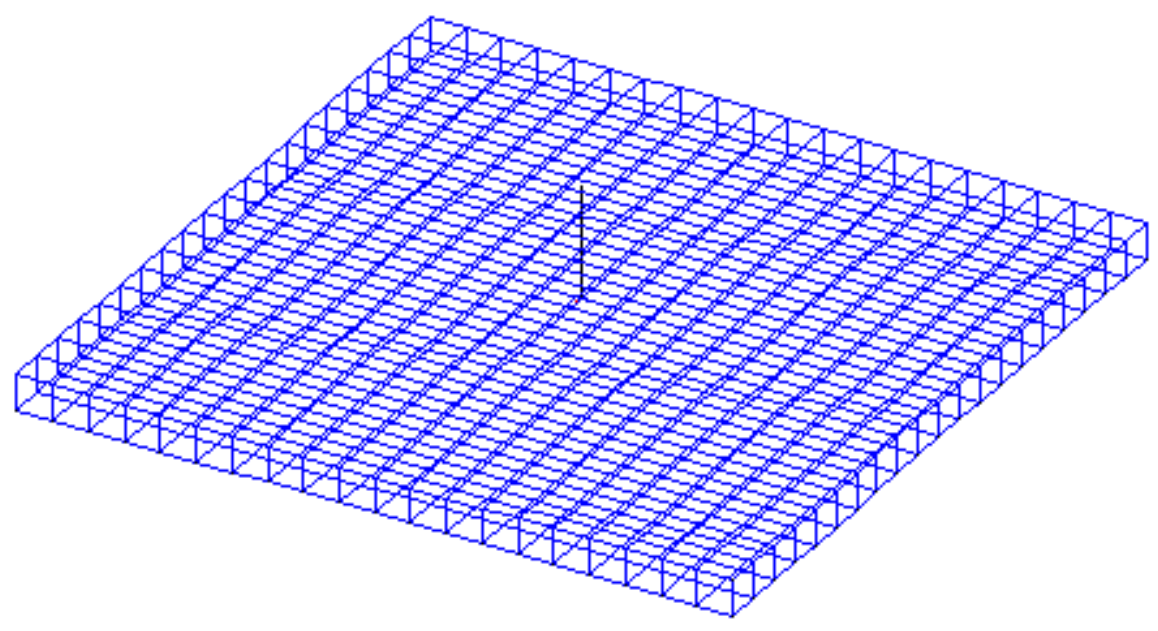

Figure 7 Closed Patch Surfaces

IV. Results

The results of the relative impedance change calculated for the different surface models have been plotted in Figure 8. It has been plotted the same way as Figure 4. The horizontal axes correspond to $\Delta R / R_{0}$, while the vertical axes correspond to $\Delta X / R_{0}$. By comparing the curves on these figures, it can easily be seen that the NEC result with the thin patch surface model agrees better with Figure 4, which is based on the numerical results obtained by Vogler and Noble [12]. Both indicate that as the distance between the antenna and the conducting surface increases, the relative impedance change decreases along a spiral curve, which is shrinking towards the origin. In Figure 4, value of $\Psi$ depends on the value of conductivity and permittivity of the conducting surface. It means that the gradient of the spiral curve varies with different kinds of conducting surfaces in the vicinity. It provides a reason for the difference between the spirals on (b) of Figure 8 and Figure 4. 


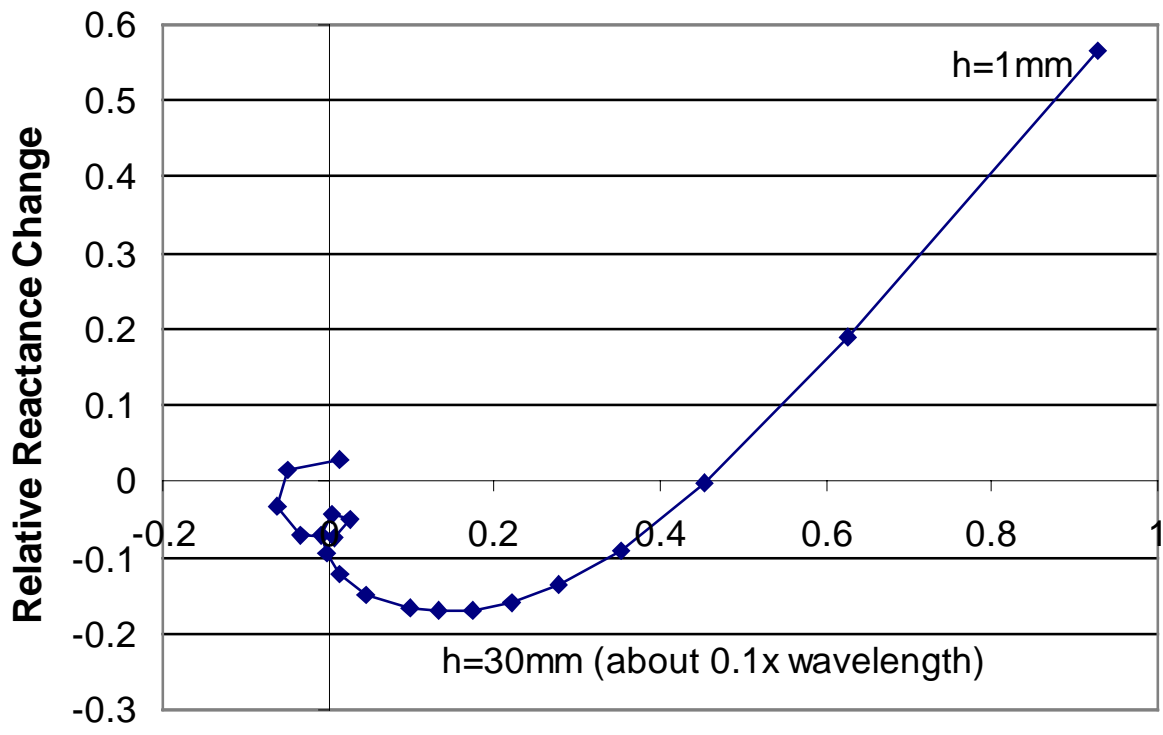

Relative Resistance Change

(a) Surface Model with Wire-Grid

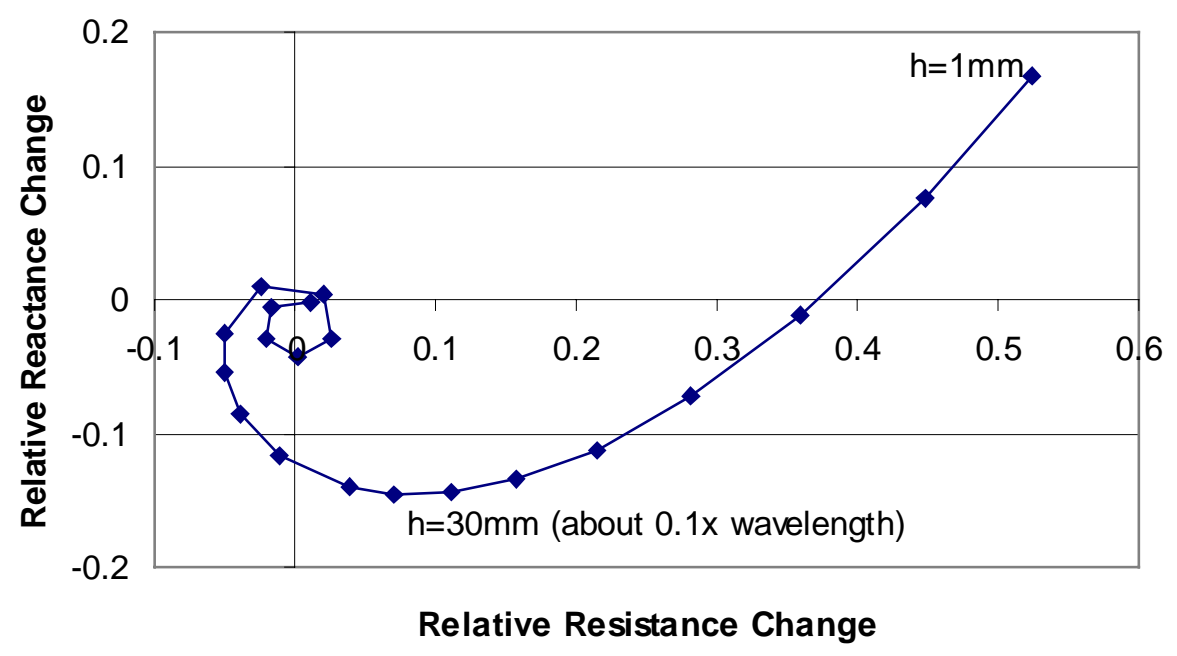

(b) Surface Model with Thin Patch Surface 


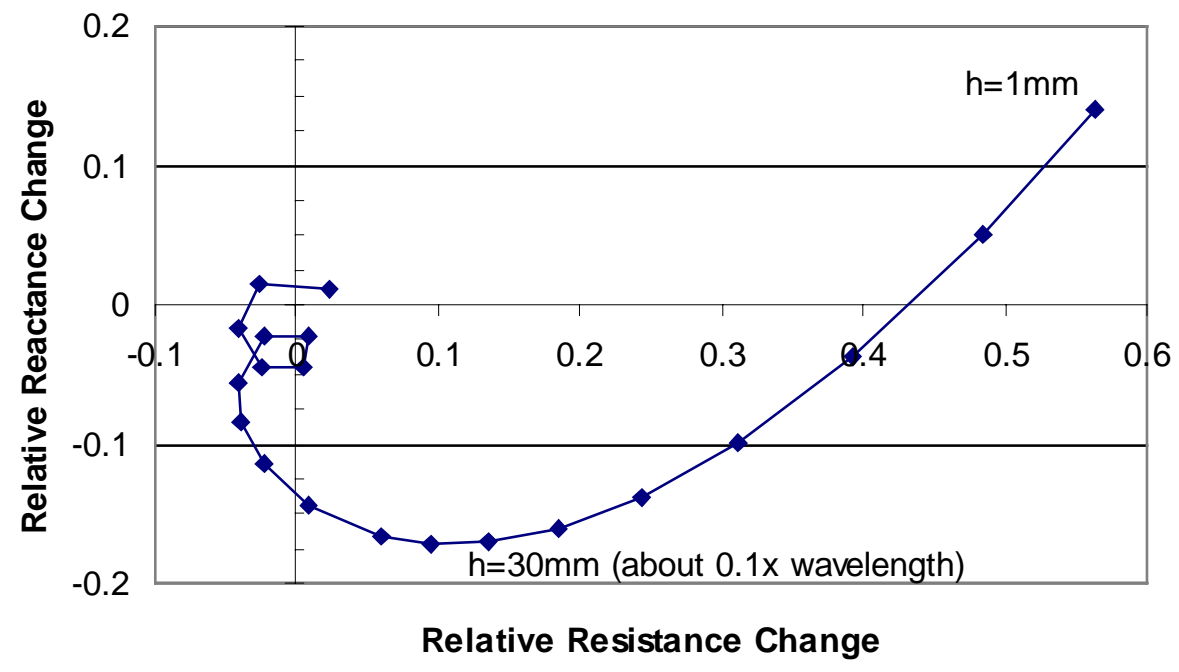

(c) Surface Model with Closed Patch Surfaces

Figure 8 Relative Impedance Change of the Vertical Dipole over the 3' x 3' Metal Plate Modeled with Different Surface Elements

The time spent calculating each point on the curve varies among different models. The wire-grid model takes about $1,360 \mathrm{sec}$, the 20x20 thin patch model takes about $94 \mathrm{sec}$, and the closed patch surfaces model takes about $956 \mathrm{sec}$.

Considering that there is always a compromise between the calculation time and the accuracy, various numbers of patch segments have been used for modeling the thin patch surface. Figure 9 illustrates the results from discretizing the $3 \mathrm{ft} \times 3 \mathrm{ft}$ plate by 10x10, 20x20, and $40 \times 40$.

The calculation of each point on the curve takes $3.7 \mathrm{sec}$ for 10x10, $94 \mathrm{sec}$ for $20 \times 20$, and 11,697 sec for 40x40. The result from the 20x20 agrees well with that from the 40x40, while saving quite a significant amount of time. Consequently, the 20x20 patch segment was chosen to model the metal plate for this study. 


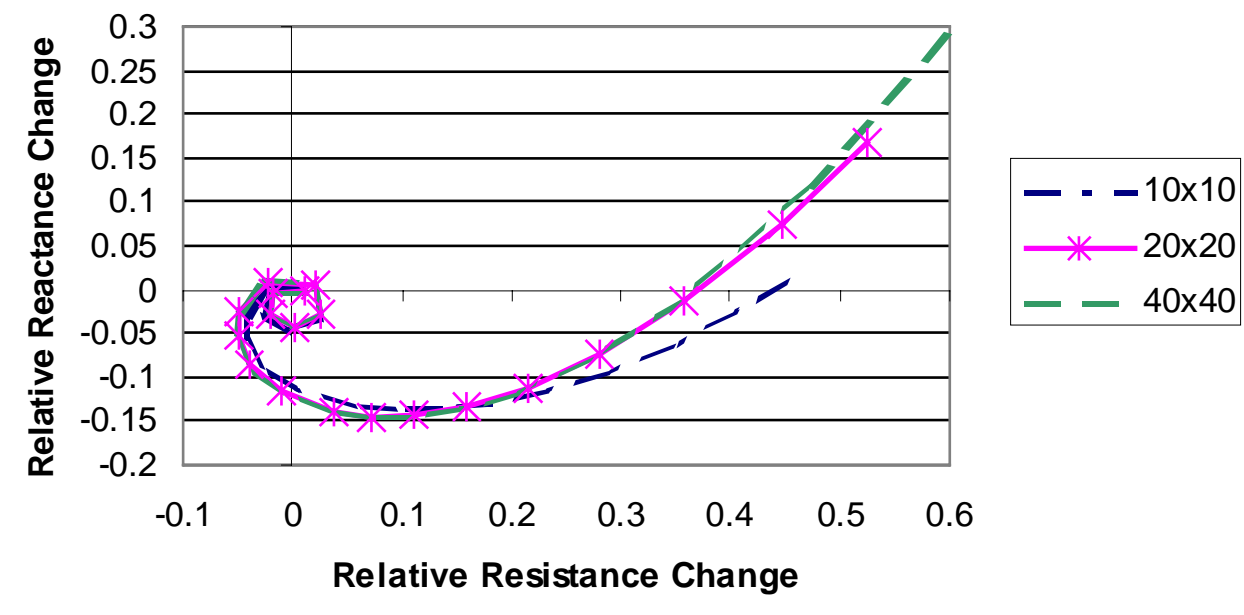

Figure 9 Vertical Dipole Numerical Results for Different Plate Discretization

\section{2) Horizontal Dipole over 3ft x 3ft Metal Plate}

The horizontal dipole is modeled to be over a 20x20 thin patch surface. Its resistance and reactance change as the height of it over the metal plate varies. Figure 10 exhibits its resistance and reactance in a function of the height relative to the free space wavelength. The numerical result of its relative impedance change is shown in Figure 17 in Chapter 4.

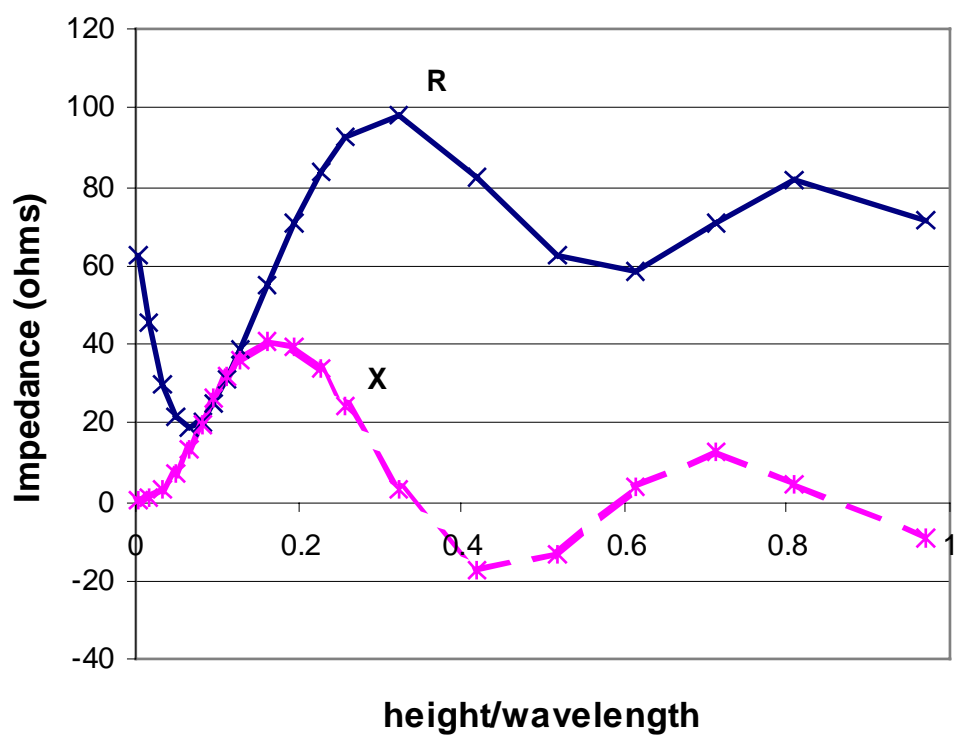

Figure 10 Input Impedance of Horizontal Dipole in a Function of Relative Height 
Figure 10 and Figure 3 have extremely similar contours, except that the reactance curve in Figure 10 crosses the horizontal axis because the impedance is calculated at its resonance frequency, while Figure 3 shows the result at a frequency other than resonance frequency. Both figures indicate that as the height of the horizontal dipole over the conducting surface increases, both its resistance and reactance oscillate around the value of free space impedance, namely 72 ohms of resistance, and $0 \mathrm{ohms}$ of reactance. The amplitude of oscillation decreases as the height increases.

\section{3) Horizontal Loop over 3ft $x$ 3ft Metal Plate}

The NEC result for the relative impedance change of the horizontal loop over a 20x20 thin patch surface model is illustrated in Figure 19, and the loop's resistance and reactance as a function of the relative height to the free space wavelength is plotted in Figure 20 in Chapter 4.

\section{4) Horizontal CTHA over 3ft $x$ 3ft Metal Plate}

Only the wires of CTHA have been modeled; the core material made of high density polyethylene, on which the wires are wound, could not be modeled with NEC, because it does not provide the function for modeling a core material with a dielectric constant other than one, which corresponds to air. However, it is possible to model different grounds with different material properties.

Using feed 6 [13], CTHA is not naturally matched. Consequently its impedance change has been studied at a high resistance resonance frequency ( $3^{\text {rd }}$ resonance), a low resistance resonance frequency $\left(4^{\text {th }}\right.$ resonance), and off the $3^{\text {rd }}$ resonance frequency when resistance is about 50 ohms. The results of the relative impedance change are shown in Figure 21 in Chapter 4. Figure 22 illustrate its resistance and reactance as a function of relative height to the free space wavelength. 
Table 4 Free Space Frequencies and Impedance of the CTHA in NEC Analysis

\begin{tabular}{|l|c|c|c|}
\hline & $3^{\text {rd }}$ Resonance & off $3^{\text {rd }}$ Resonance & $4^{\text {th }}$ Resonance \\
\hline Frequency (MHz) & 917.21 & 937.5 & 1215 \\
Resistance (ohms) & 35843 & 51.05 & 0.456 \\
Reactance (ohms) & 17.4 & -1345.8 & 0.636 \\
\hline
\end{tabular}

\section{Summary}

This chapter has primarily described the modeling of antennas above metal plates with NEC. The results for different models have been analyzed and compared with references. From the above discussion, the following points can be made:

- The thin patch surface is a reasonable approach for modeling the metal plate in this impedance study. Instead of representing the current flowing on the surface by the current flowing in the wires of a mesh, it represents the current as flowing on the surface in two orthogonal directions on square flat patches. The surface patch gives a more accurate representation of a surface, especially for near-field quantities, than a wire mesh because it directly models the surface and there are no problems relating to equivalence of the sort mentioned for wire meshes [14]. For calculation of the input impedance of the antennas, closed patch surfaces have not displayed much more accuracy than thin patch surfaces while taking about ten times more time to solve.

- By comparing the results given by NEC with the results of related research, it is evident that the NEC results provide a reasonable prediction of the impedance change as the distance between the antenna and the metallic ground plane changes. 
- From the numerical results, it could be found that the relative impedance change of the horizontal CTHA follows the similar spiral curves as the vertical dipole, the horizontal dipole, and the horizontal loop. It decreases as the distance between the CTHA and the metal plate increases.

- For the same object in the vicinity of an antenna, its effect on the impedance of different antennas is different. Further comparison of the impedance effect on different antennas will be discussed in Chapter 6. 


\section{Chapter 3 Measurement of Antennas' Input Impedance}

In Chapter 2, the NEC modeling and results for the $3 \mathrm{ft} \times 3 \mathrm{ft}$ metal plate study have been discussed. To further evaluate the accuracy of the NEC results, the measurements of the antennas' impedance have been conducted and the NEC results will be compared with the experimental results in Chapter 4.

\section{Introduction of Equipment}

\section{1) Anechoic Chamber}

Above discussion has indicated that objects in the vicinity of an antenna affect the input impedance of the antenna. Generally, the impedance change is a combined result of the interaction of different objects. In order to understand this effect, it is of primary importance to study the effect of each object separately. The metal plate experiment is designed to study the effect of a metal plate on the input impedance change of antennas. To screen out the influence by the ground and other adjacent objects, an anechoic chamber is used for the experiment.

An anechoic (no echo) chamber is constructed by completely covering all room surfaces with absorbing material. "The philosophy is to provide a nonreflecting environment like in outer space, but with the distinct advantage that the room provides shielding from all of the external electromagnetic noise and interference (natural and man-made)" [15].

The Center for Industrial Research Applications (CIRA) at West Virginia University has constructed an anechoic chamber to measure antenna characteristics in free space conditions. The dimensions of the chamber are $8 \mathrm{ft} \times 8 \mathrm{ft} \times 18 \mathrm{ft}$ wooden box with $1 \mathrm{ft}$ thick foam cones, resulting in a cone tip-to-tip distance of $6 \mathrm{ft} \times 6 \mathrm{ft} \times 16 \mathrm{ft}$. The walls are covered with EHP-12 
wedge absorber material supplied by RANTEC (the properties of the absorber material are shown in Appendix C). Figure 11 shows the inside view of the WVU anechoic chamber. As indicated in Pertl's report about the validation of the anechoic chamber [16], the effectiveness of the chamber for simulating a free space environment is acceptable when operating at a frequency above $900 \mathrm{MHz}$.

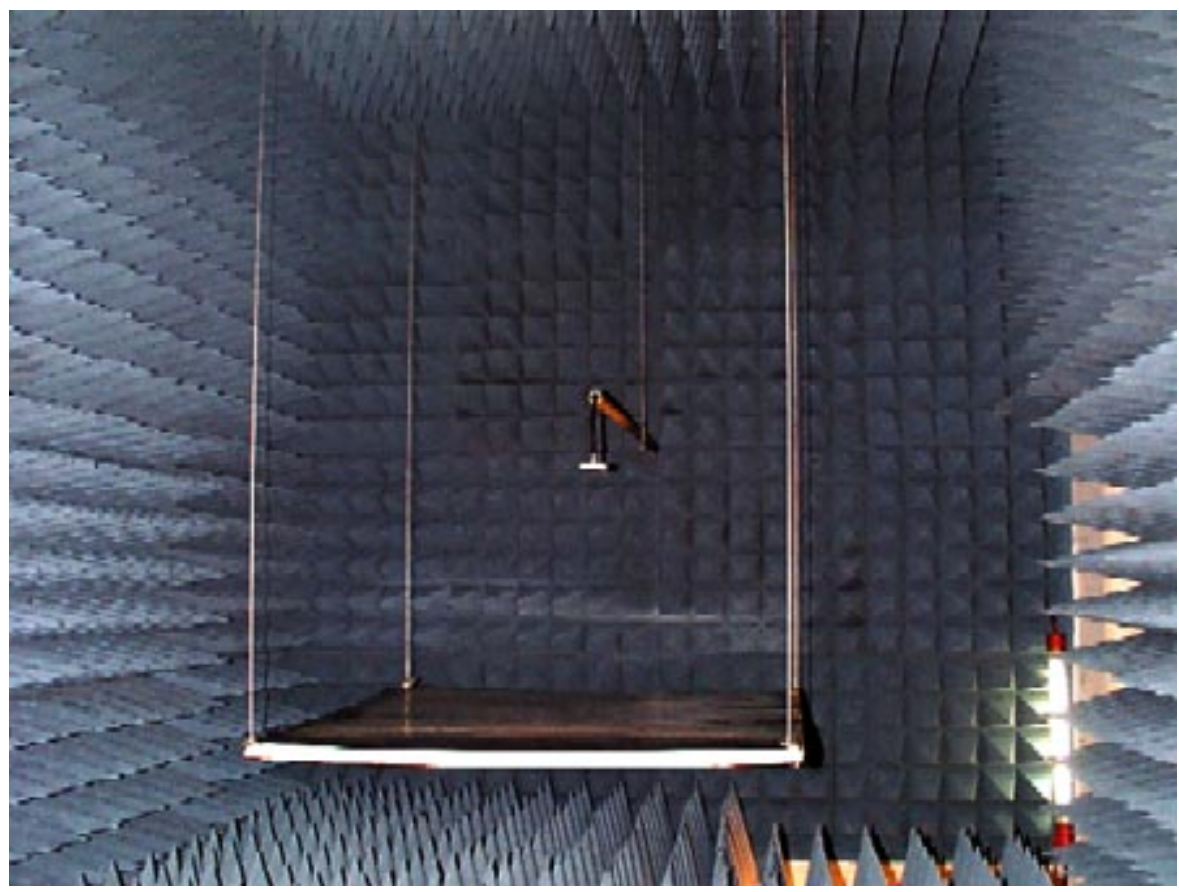

Figure 11 3'x3' Metal Plate under the CTHA inside the CIRA Anechoic Chamber

\section{2) HP 8753D Network Analyzer}

The HP 8753D option 011 is a high performance vector network analyzer for laboratory or production measurements of reflection and transmission parameters [17]. It provides combined digital signal processing and microprocessor controls for easy operation and measurement improvement. It is selected to measure the input impedance of antennas for the metal plate study.

The measurement results of the HP 8753D could be stored in three kinds of format: raw data, data (raw data with error-correction applied), and format (data processed to the display format). 
The raw data array has the least amount of processing associated with it, while the format array is the result after corrections, electrical delay, and other processings. In this experiment, the Port Extension function is applied to calibrate out the SMA connector of antenna (details are discussed in part 4 "Measurement Calibration"). This function involves electrical compensation, so the format data is used.

Within the format data file (".F1" for channel 1 or ".F2" for channel 2), the impedance results are stored in real and imaginary data pairs (Re and $\mathrm{Im})$. For interpreting the real and imaginary data pair into resistance and reactance, the following formula is applied:

$$
\begin{aligned}
& \text { Resistance }=\left(1-R e^{2}-I^{2}\right) /\left((1-R e)^{2}+I m^{2}\right) \times Z_{0} \\
& \text { Reactance }=(2 \times I m) /\left((1-R e)^{2}+I m^{2}\right) \times Z_{0}
\end{aligned}
$$

where $\mathrm{Z}_{0}$, the characterized impedance of the system, is $50 \mathrm{ohms}$ in this experiment.

\section{3) Setup of the 3ft $x$ 3ft Metal Plate Study}

The setup of the test is as shown in Figure 11, Figure 12 and Figure 13. The central axis of the metal plate crosses the center of the antenna being tested. The level of the plate was checked before the start of the tests, and it was maintained through keeping the same length of the four pieces of strings hanging the plate throughout the tests. For testing all the antennas, impedance measurement started from the smallest distance $(1 \mathrm{~mm})$. The distance between the antenna and the metal plate is increased through loosing the strings controlled outside the anechoic chamber.

Each antenna was connected to a coax cable through an SMA connector. The coax cable connected the antenna inside the anechoic chamber with the network analyzer outside the chamber. 

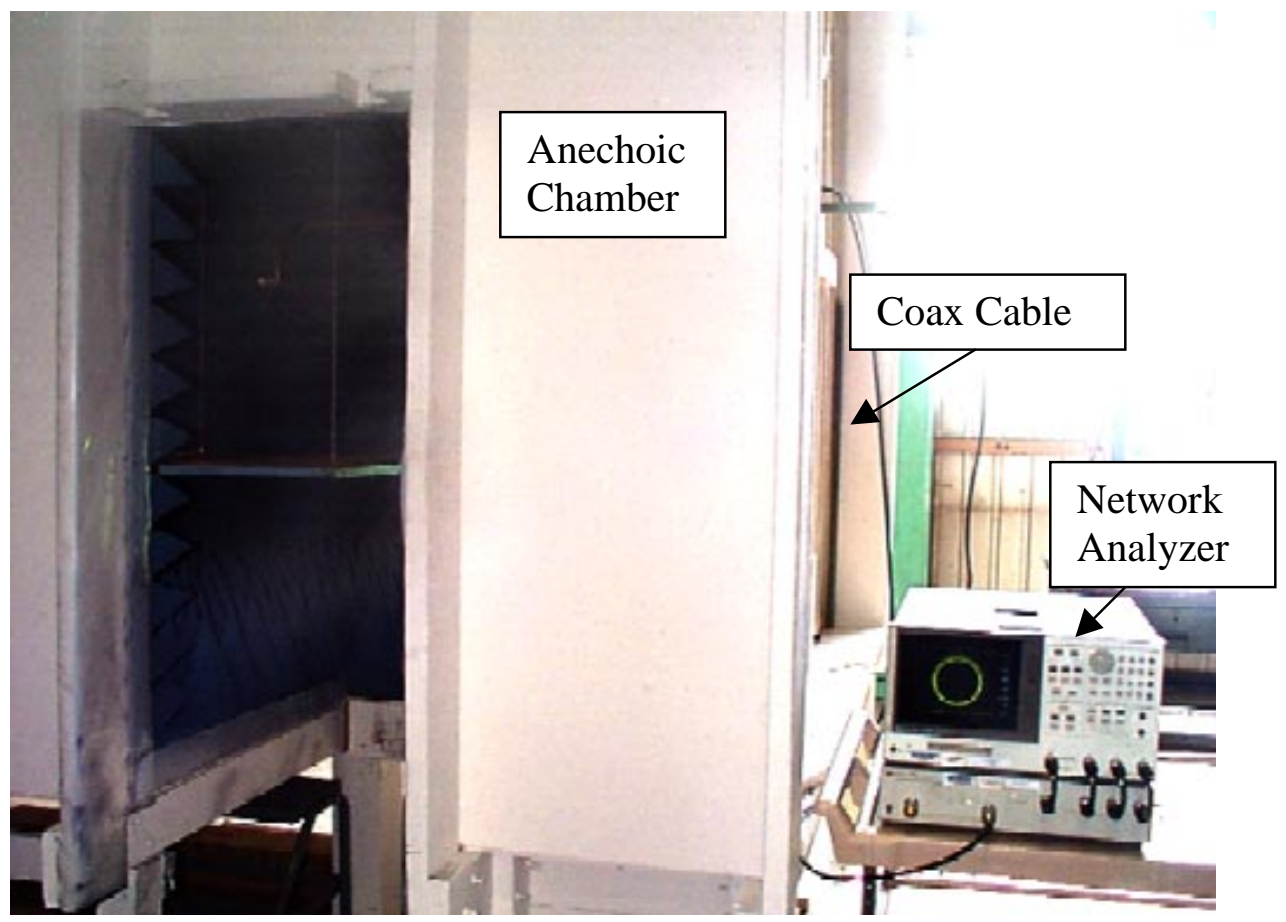

Figure 12 Picture of the Testing Site

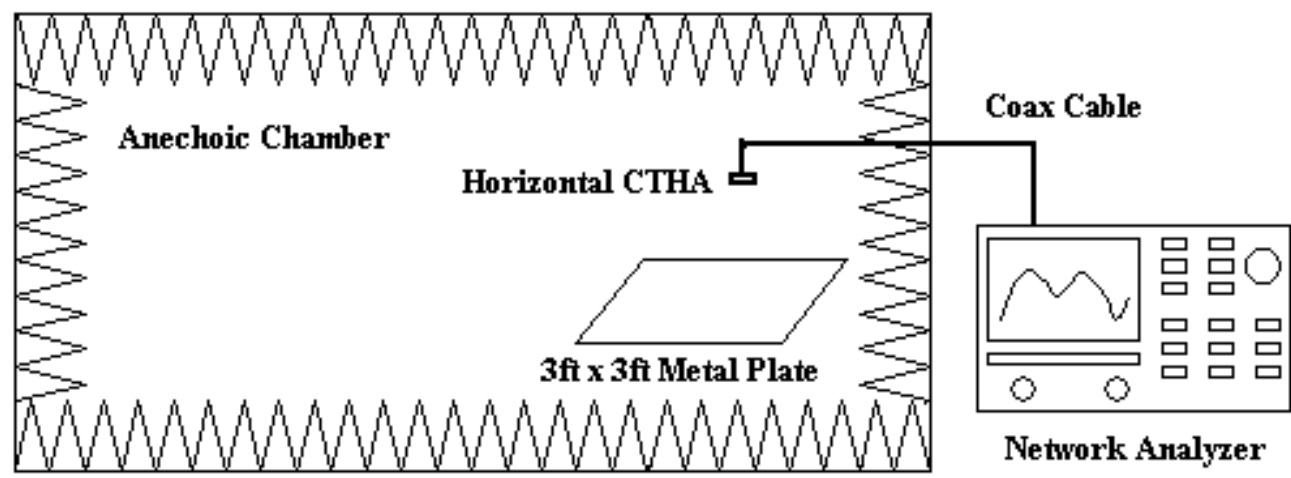

Figure 13 Diagram of the Setup of Impedance Measurement

\section{4) Measurement Calibration}

In this metal plate experiment, the antenna input impedance is measured. However, the unknown impedance is inevitably connected to the measurement device with a length of cable. The actual impedance being measured is not the unknown impedance-it is the impedance of the 
connection cable that is terminated in the unknown impedance [18]. To measure only the desired unknown impedance of the antennas, the connection cable needs to be calibrated out.

"Measurement calibration is an accuracy enhancement procedure that effectively removes the system errors that cause uncertainty in measuring a test device. It measures known standard devices and uses the results of these measurements to characterize the system" [17]. When the calibration includes the connection cable as a part of the measuring system, the cable can be calibrated out as desired. The known standard device used to calibrate the measuring system in this experiment is an HP85033D 3.5mm female calibration kit. The frequency range of $400 \mathrm{MHz}$ to $1.6 \mathrm{GHz}$ was selected (the frequency range of the instrument is from $30 \mathrm{KHz}$ to $6 \mathrm{GHz}$ ), and 1601 points were set within this range.

Considering that antennas are generally used without connectors, and that the numerical models discussed above exclude the SMA connector used to connect the test antennas to the connection cable in this experiment, the Port Extension feature of the network analyzer has been applied. This feature compensates for the phase shift of the extended measurement reference plane due to the SMA connector so that the effect of the SMA connector on the antenna's impedance measurement can be canceled out. 


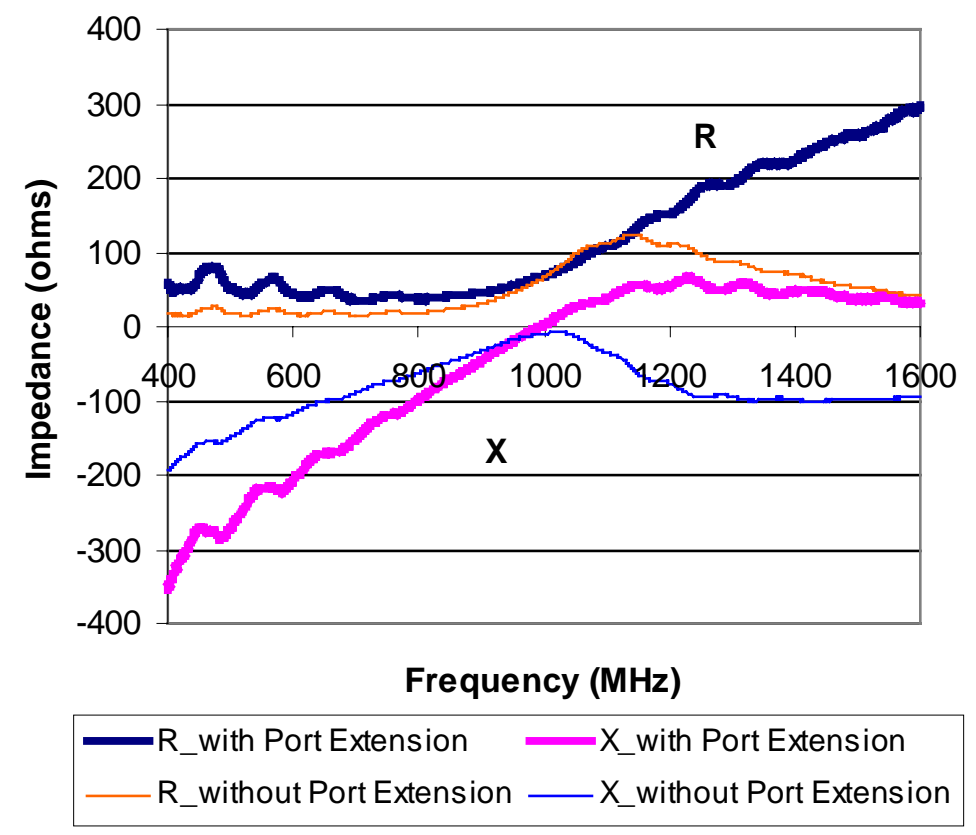

Figure 14 Measured Free Space Impedance of a Half-Wave Dipole

Figure 14 shows the measured free space impedance of a half-wave dipole with and without the Port Extension. From the results, no crossing could be found on the reactance curve with the zero-axis without activating the Port Extension. In other words, no resonance is detected around the designed working frequency. This result does not agree with common knowledge about halfwave dipoles. Consequently using the Port Extension to cancel out the effect of the SMA connector on the measurement result of antenna impedance is necessary.

\section{Experimental Results}

The antennas modeled in Chapter 2, the vertical dipole, the horizontal dipole, the horizontal loop, and the horizontal CTHA, have been tested. The experimental results are shown in Chapter 4. The frequencies are selected for the impedance change study according to the free space measurement result so that the impedance study will focus on the case that antenna is at or close 
to resonance. Table 5 lists the free space frequencies and impedance at the frequencies for both the numerical calculations and the experiments.

Table 5 Free Space Frequencies and Impedance

\begin{tabular}{|c|c|c|c|c|c|c|}
\hline & \multicolumn{3}{|c|}{ Numerical Calculation by NEC } & \multicolumn{3}{|c|}{ Experiment } \\
\hline & $\begin{array}{l}\text { Freq. } \\
(\mathrm{MHz})\end{array}$ & $\begin{array}{c}\mathrm{R} \\
\text { (ohms) }\end{array}$ & $\begin{array}{c}\mathrm{X} \\
\text { (ohms) }\end{array}$ & $\begin{array}{l}\text { Freq. } \\
(\mathrm{MHz})\end{array}$ & $\begin{array}{c}\mathrm{R} \\
\text { (ohms) }\end{array}$ & $\begin{array}{c}\mathrm{X} \\
\text { (ohms) }\end{array}$ \\
\hline Vert. Dipole & 971 & 71.89 & -0.01 & 994.75 & 67.24 & 0.14 \\
\hline Hori. Dipole & 971 & 71.89 & -0.01 & 994.75 & 68.71 & -0.08 \\
\hline Hori. Loop & 1035 & 142.92 & 0.02 & 1026.25 & 131.61 & 0.32 \\
\hline $\begin{array}{l}\text { CTHA } \\
3^{\text {rd }} \text { Resonance }\end{array}$ & 917.21 & 35843 & 17.4 & 739 & 575.17 & 10.38 \\
\hline $\begin{array}{l}\text { CTHA } \\
\text { R 50ohms }\end{array}$ & 937.5 & 51.05 & -1345.8 & 841 & 49.7 & -147.8 \\
\hline $\begin{array}{l}\text { CTHA } \\
4^{\text {th }} \text { Resonance }\end{array}$ & 1215 & 0.46 & 0.64 & 952 & 1.35 & -0.27 \\
\hline
\end{tabular}

Table 6 Percentage Difference of Numerical Result to Experimental Result

\begin{tabular}{|c|c|c|c|}
\hline Relative Error & Frequency & Resistance & Reactance \\
\hline Vertical Dipole & $-2.4 \%$ & $6.9 \%$ & $*$ \\
\hline Horizontal Dipole & $-2.4 \%$ & $4.6 \%$ & $*$ \\
\hline Horizontal Loop & $0.85 \%$ & $8.6 \%$ & $*$ \\
\hline CTHA $3^{\text {rd }}$ Resonance & $24 \%$ & $6100 \%$ & $*$ \\
\hline CTHA R 50ohms & $11.5 \%$ & $* *$ & $-810 \%$ \\
\hline CTHA $4^{\text {th }}$ Resonance & $27.6 \%$ & $-66 \%$ & $*$ \\
\hline
\end{tabular}

* For resonance evaluation, taking both experimental and numerical result of reactance as 0 ** For R 50ohms, taking both experimental and numerical result of resistance as 50ohms.

From Table 6, it can be seen that the measurement parameters of the vertical dipole, horizontal dipole, and horizontal loop are close to their numerical results. But the numerical 
results of CTHA are different from the measurements because the high-density polyethylene core of CTHA could not be modeled with NEC. The result indicates that the core material of CTHA significantly affects the input impedance of the CTHA.

\section{Error Analysis of Impedance Measurement}

- Measuring Error of HP8753D Network Analyzer

For measurement in the range of $400 \mathrm{MHz}$ to $1.6 \mathrm{GHz}$ with 1,601 points, the accuracy of the resonance frequency is $\pm 0.4 \mathrm{MHz}$.

The precision of the measurement with the HP8753D network analyzer also depends on the specifications as shown in Appendix D.

- Error of Distance Measurement

The distance between the antenna and the metal plate is measured with measuring tape. Its accuracy is $\pm 0.5 \mathrm{~mm}$. Considering the error with the flatness of the plate and the shape error with the antennas, the overall error with distance measuring is $\pm 1 \mathrm{~mm}$

- Fabrication Error with Antennas

All the antennas are hand-made. Error could result from the length of wire $( \pm 0.5 \mathrm{~mm})$, soldering between the antenna and the SMA connector, and shape irregularity. 


\section{Chapter 4 Comparison of NEC and Experimental Results}

\section{Introduction}

Chapter 2 and Chapter 3 discussed the study of the impedance effects of the $3 \mathrm{ftx} 3 \mathrm{ft}$ metal plate on the CTHA and some other antennas by numerical approach and experimental approach, respectively. This chapter compares the NEC and experimental results.

With the development of computer technology, calculation speed and computer memory have been greatly improved, which allows extensive application of a computerized analytical method. NEC is one of the more recently developed programs for analyzing the electromagnetic response of antennas and scatterers. The computerized analytical method can be used to analyze not only ready-made designs, but also the future designs. If the results of the method are demonstrated to be close to reality, new designs can be optimized or evaluated numerically, saving time and money when generating ideal designs. With this consideration, the accuracy of the NEC results is evaluated by comparing the numerical results with the experimental results.

\section{Vertical Dipole}

As can be seen from Figure 15 and Figure 16, the NEC results for the vertical dipole agree well with the experimental results. The standard deviations of the numerical results to the experimental results calculated as Eq. 4-1 are $6.6 \mathrm{ohms}$ and $1.8 \mathrm{ohms}$ for resistance and reactance, respectively. The correlation coefficients calculated as Eq. 4-2 are 0.99 and 0.95 for resistance and reactance, respectively. 


$$
\begin{aligned}
& \text { Standard Deviation }=\sqrt{\frac{\sum_{i=1}^{n}\left(N_{i}-E_{i}\right)^{2}}{n},} \\
& \text { CorrelationCoefficient }=\frac{\frac{1}{n} \sum_{i=1}^{n}\left(N_{i}-\bar{N}\right)\left(E_{i}-\bar{E}\right)}{\sigma_{N} \sigma_{E}}, \\
& \bar{N}=\sum_{i=1}^{n} N_{i}, \\
& \bar{E}=\sum_{i=1}^{n} E_{i},
\end{aligned}
$$

According to the theory of data fitting [19], the closer to 1 the correlation coefficient is, the better the two sets of data fit each other. The correlation coefficients of the resistance and reactance of the vertical dipole demonstrate that the numerical results for the vertical dipole fit well with the experimental results.

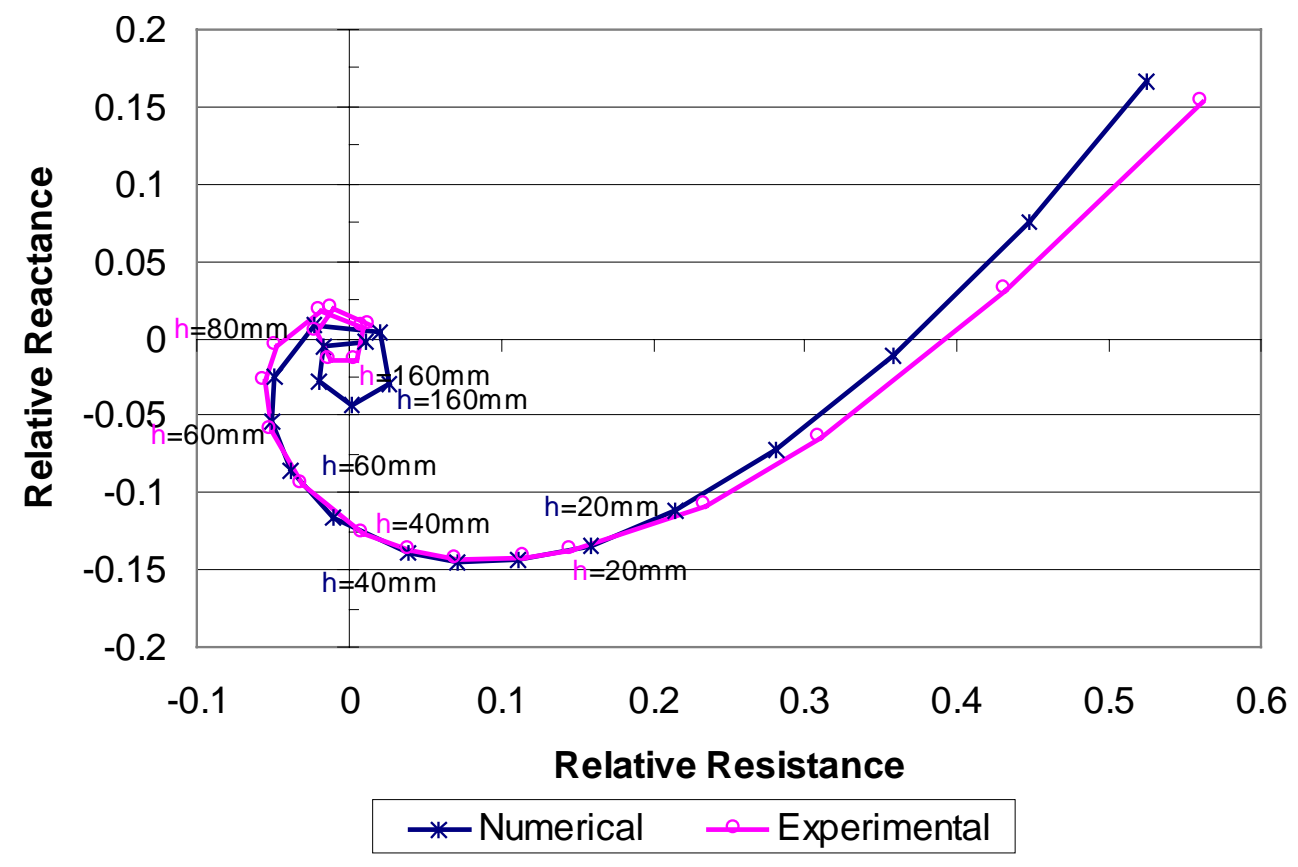

Figure 15 Relative Impedance Change of the Vertical Dipole 


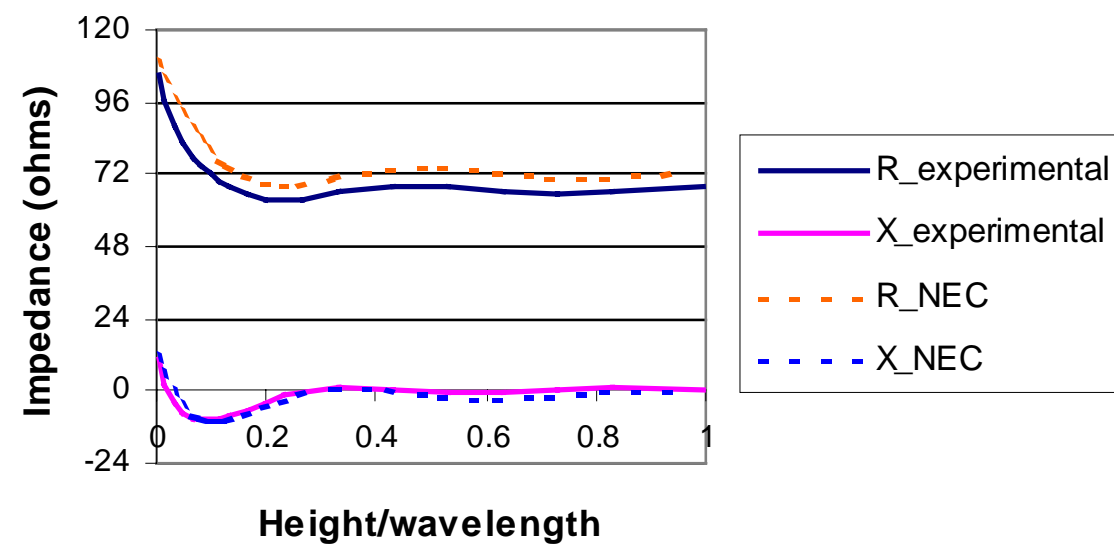

Figure 16 Resistance and Reactance of the Vertical Dipole as a Function of the Relative Height

\section{Horizontal Dipole}

Figure 17 indicates that the relative impedance change of the horizontal dipole given by NEC follows the same trend as the experimental results except that when the height is less than $20 \mathrm{~mm}$ (about $0.06 \lambda$ ). The standard deviations of numerical results to experimental results are $19.6 \mathrm{ohms}$ and $9.3 \mathrm{ohms}$ for resistance and reactance, respectively. Taking out the obviously skewed points, which correspond to the distances less than $20 \mathrm{~mm}$, the standard deviations are 8.7 ohms for both resistance and reactance. The correlation coefficients without the skewed points are 0.79 and 0.87 for resistance and reactance, respectively.

From the curves and the correlation coefficients, it is evident that the numerical results for the horizontal dipole do not fit the experimental results as well as they did for the vertical dipole. However, comparing the impedance of the vertical dipole with that of horizontal dipole, it is obvious that horizontal dipole's impedance is more variant as the height changes. It means the inaccuracy of the distance measurement may result in a larger error in the experimental results for the horizontal dipole. 


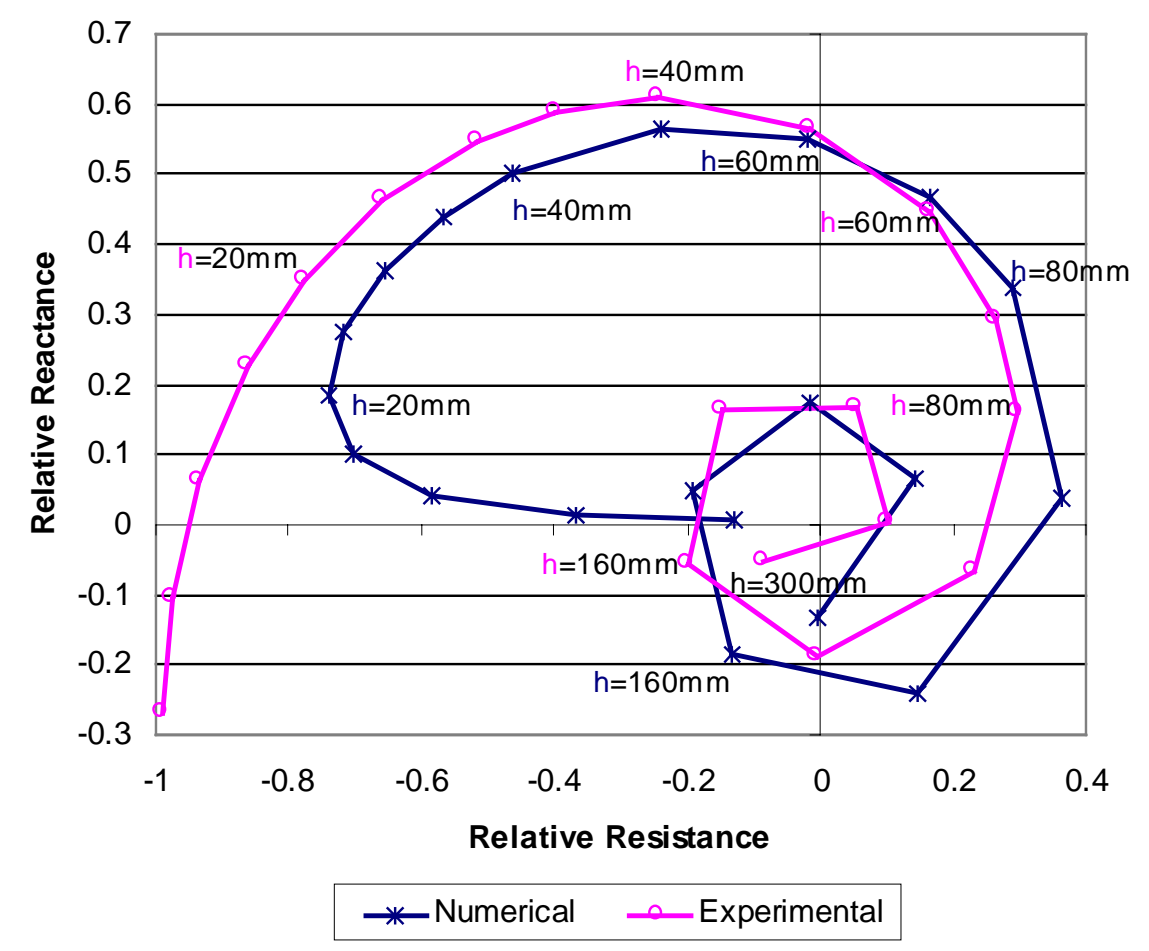

Figure 17 Relative Impedance Change of the Horizontal Dipole

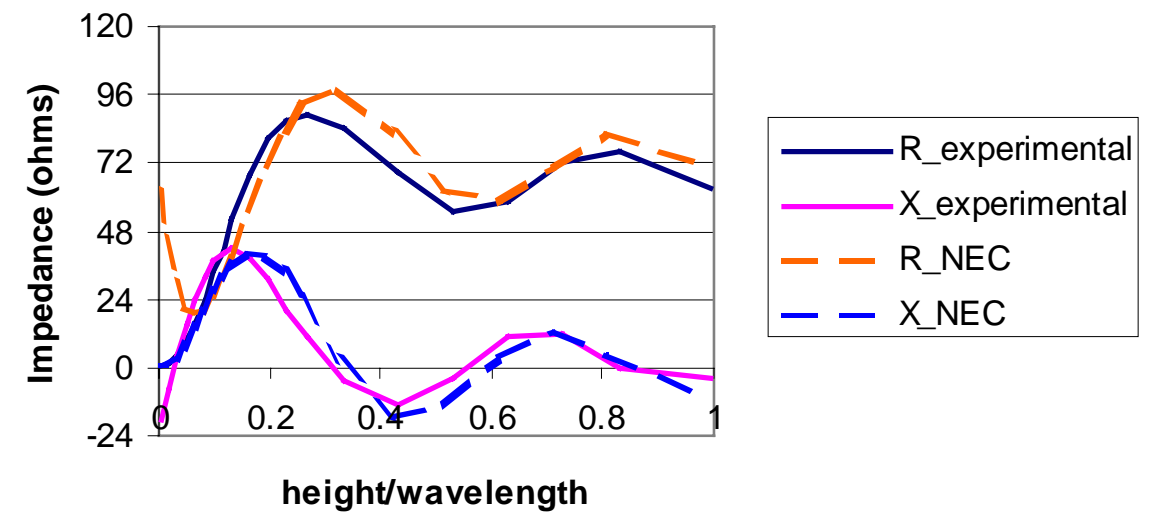

Figure 18 Resistance and Reactance of the Horizontal Dipole as a Function of the Relative Height

\section{Horizontal Loop}

Comparing Figure 19 with Figure 5, the similarity between the spirals can be observed. Comparing the NEC results with the experimental results shows that they agree with each other very well except when $\mathrm{h}<20 \mathrm{~mm}$. The standard deviations are $16.5 \mathrm{ohms}$ and $5.1 \mathrm{ohms}$ for 
resistance and reactance, respectively. The correlation coefficients are 0.99 and 0.998 for resistance and reactance, respectively. This means that when the height is larger than $20 \mathrm{~mm}$, NEC results fit the experimental results very well.

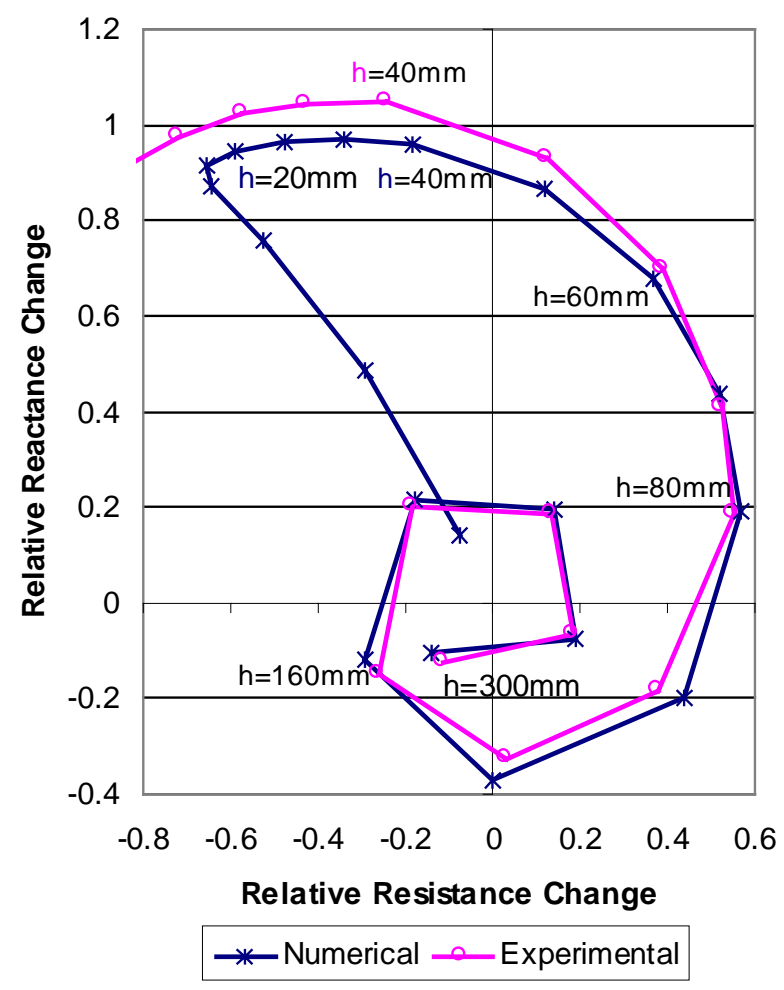

Figure 19 Relative Impedance Change of the Horizontal Loop

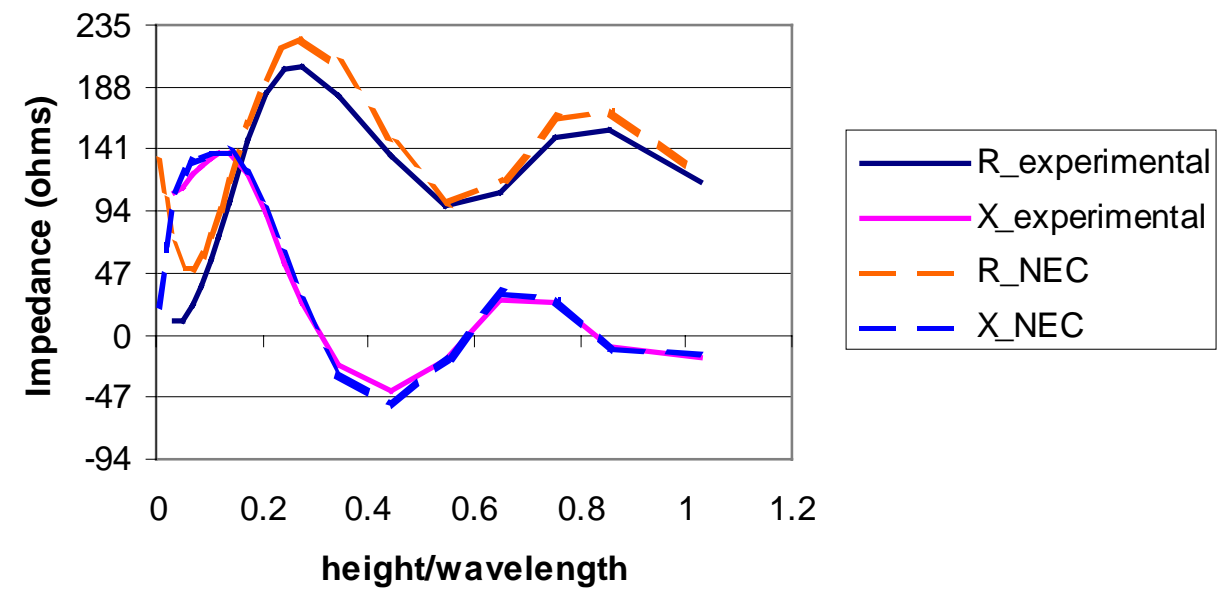

Figure 20 Resistance and Reactance of the Horizontal Loop as a Function of the Relative Height 


\section{Horizontal CTHA}

Figure 21 and Figure 22 exhibit significant differences between the NEC and experimental results for the horizontal CTHA at different frequencies. At the $3^{\text {rd }}$ resonance frequency, the standard deviations are 30,448 ohms and 7,127 ohms for resistance and reactance, respectively; when $\mathrm{R} \approx 50 \mathrm{ohms}$, they are $24 \mathrm{ohms}$ and 1,212 ohms for resistance and reactance, respectively; at the $4^{\text {th }}$ resonance frequency, they are $126 \mathrm{ohms}$ and $218 \mathrm{ohms}$ for resistance and reactance, respectively.

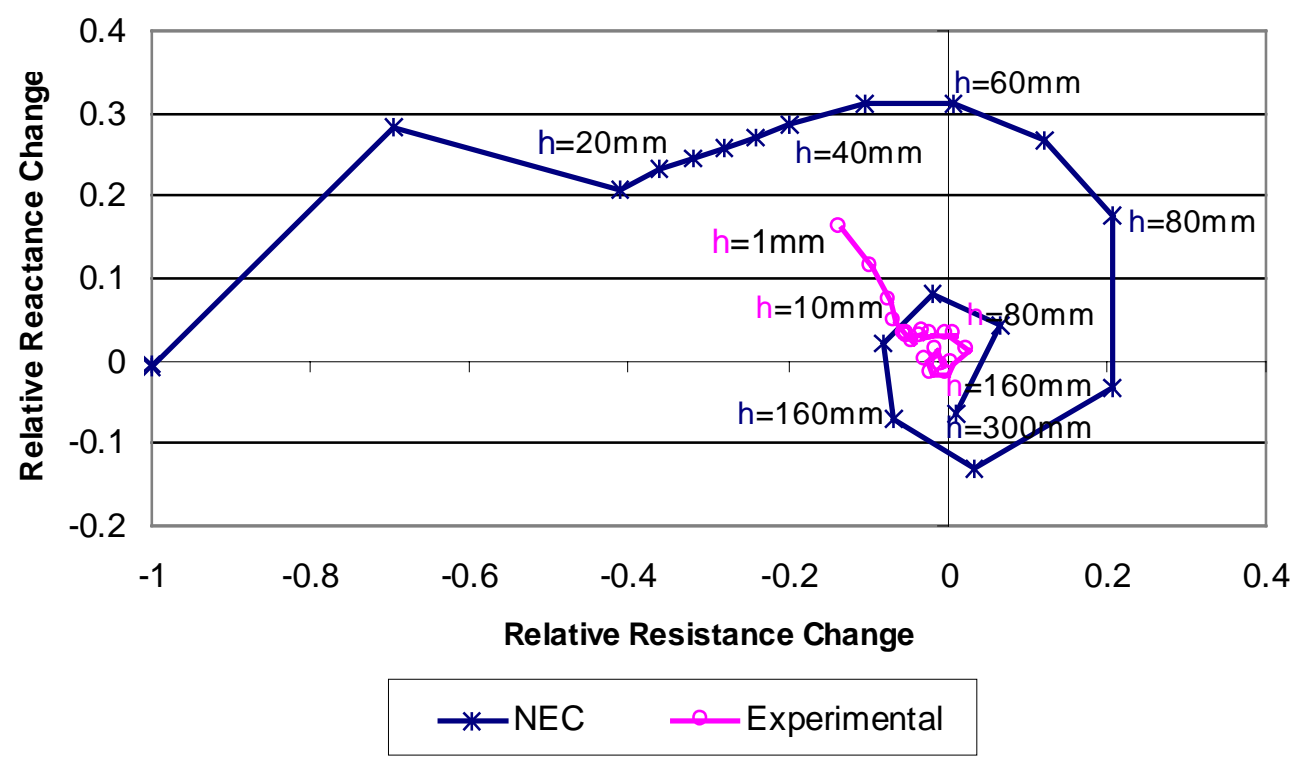

(a) at the $3^{\text {rd }}$ Resonance Frequency 


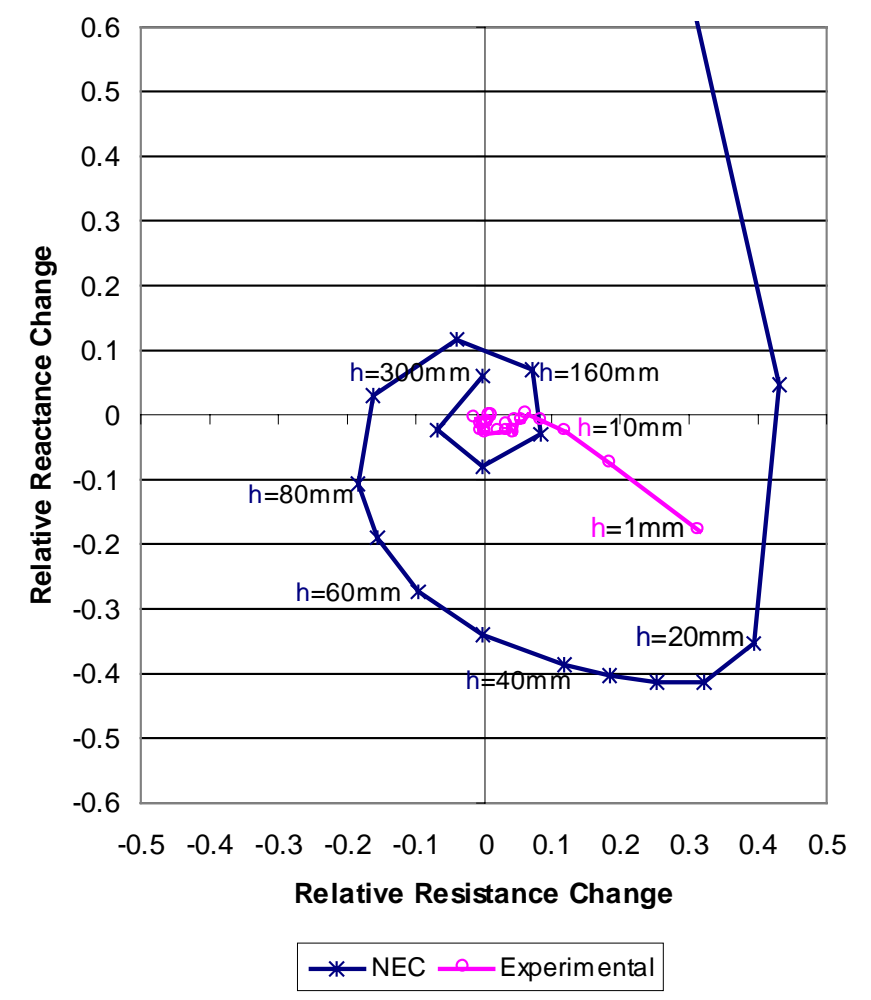

(b) off the $3^{\text {rd }}$ Resonance Frequency when $R \approx 50$ ohms

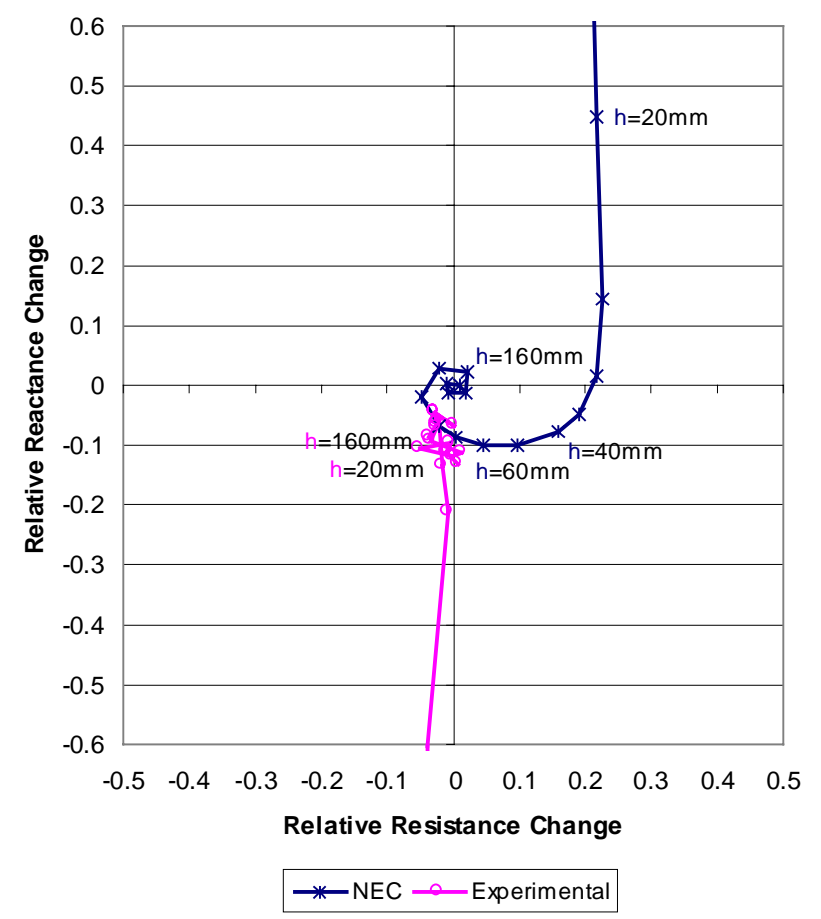

(c) at the $4^{\text {th }}$ Resonance Frequency

Figure 21 Relative Impedance Change of the Horizontal CTHA 
At the $3^{\text {rd }}$ resonance frequency and off the $3^{\text {rd }}$ resonance frequency when $R \approx 50 \mathrm{ohms}$, the NEC results have exagerated the CTHA's resistance and the amplitude of the impedance change. To get a better view of the CTHA's resistance and reactance resulting from the experiments, its experimental results at these two frequencies have been plotted separately in addition to the comparison graphs. From the similarity of the curves, it can be found that NEC has correctly predicted the tendency of the impedance change.

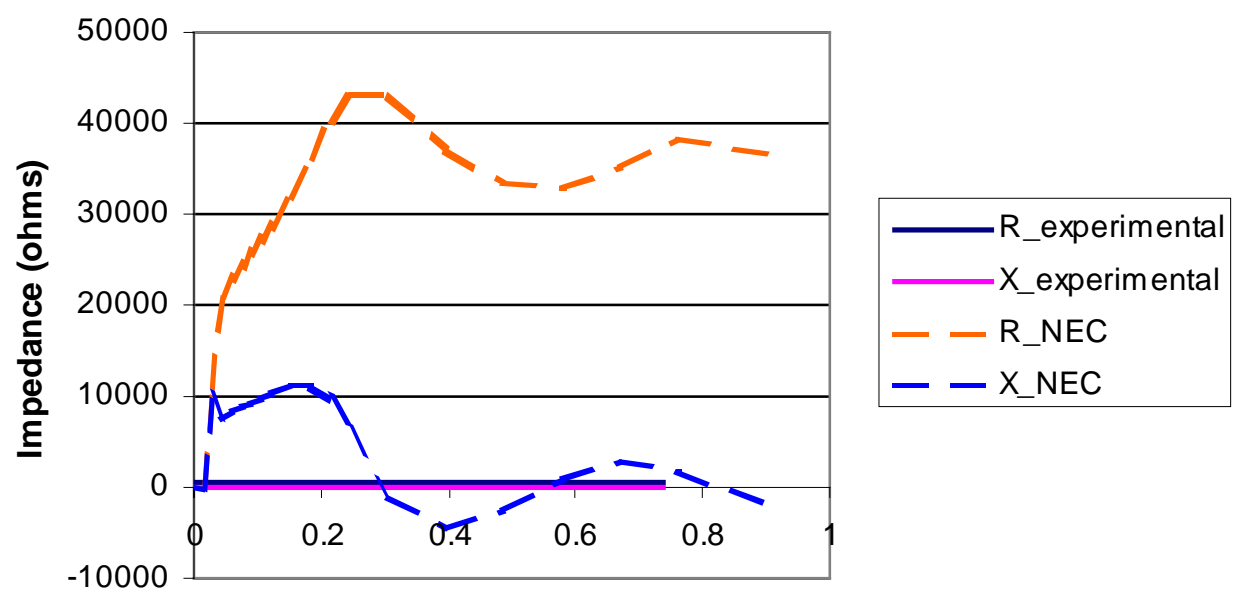

Height/wavelength

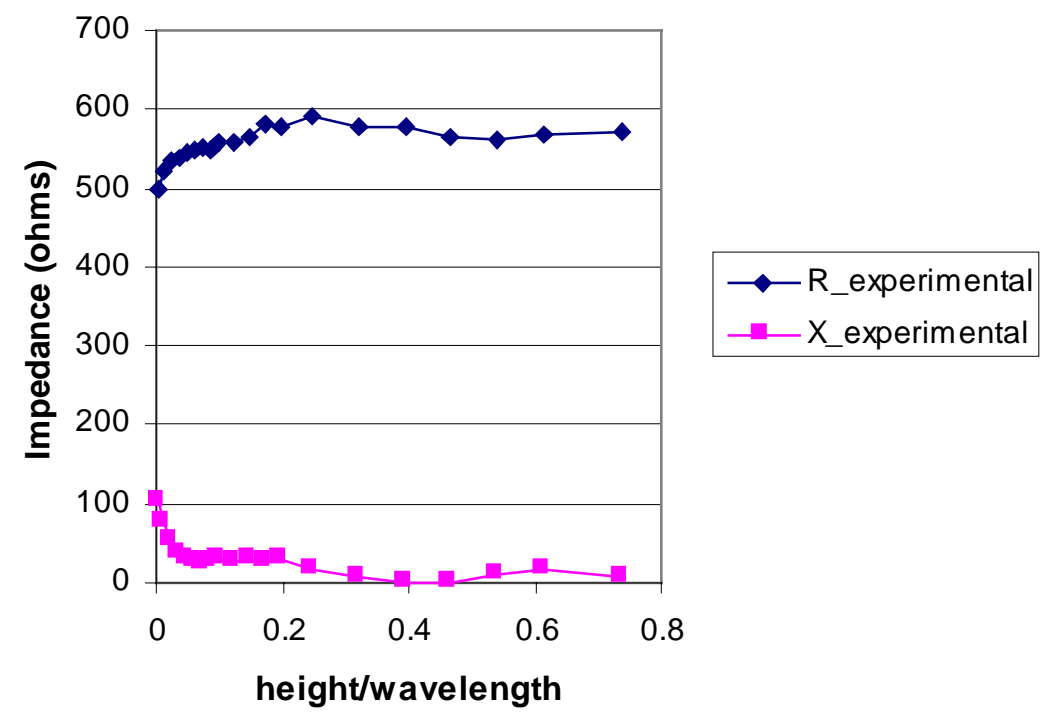

(a) at the $3^{\text {rd }}$ Resonance Frequency 


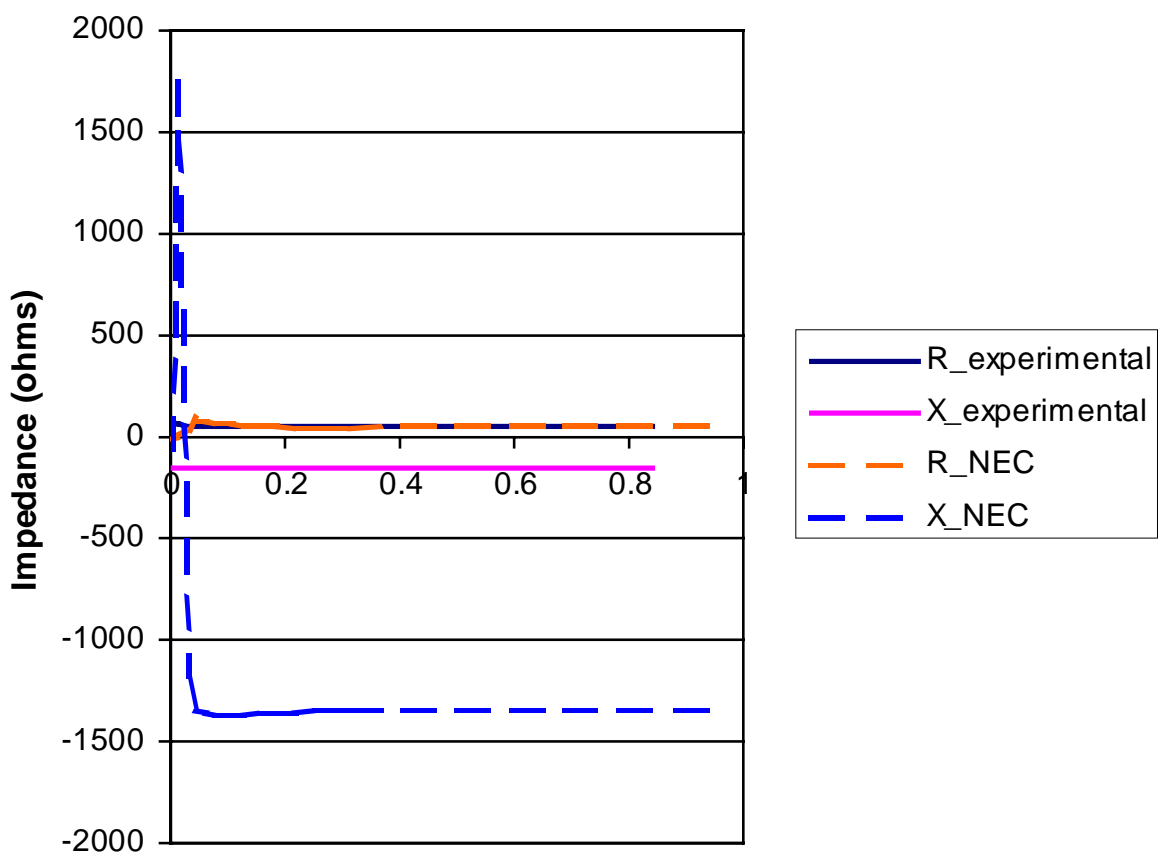

Height/wavelength

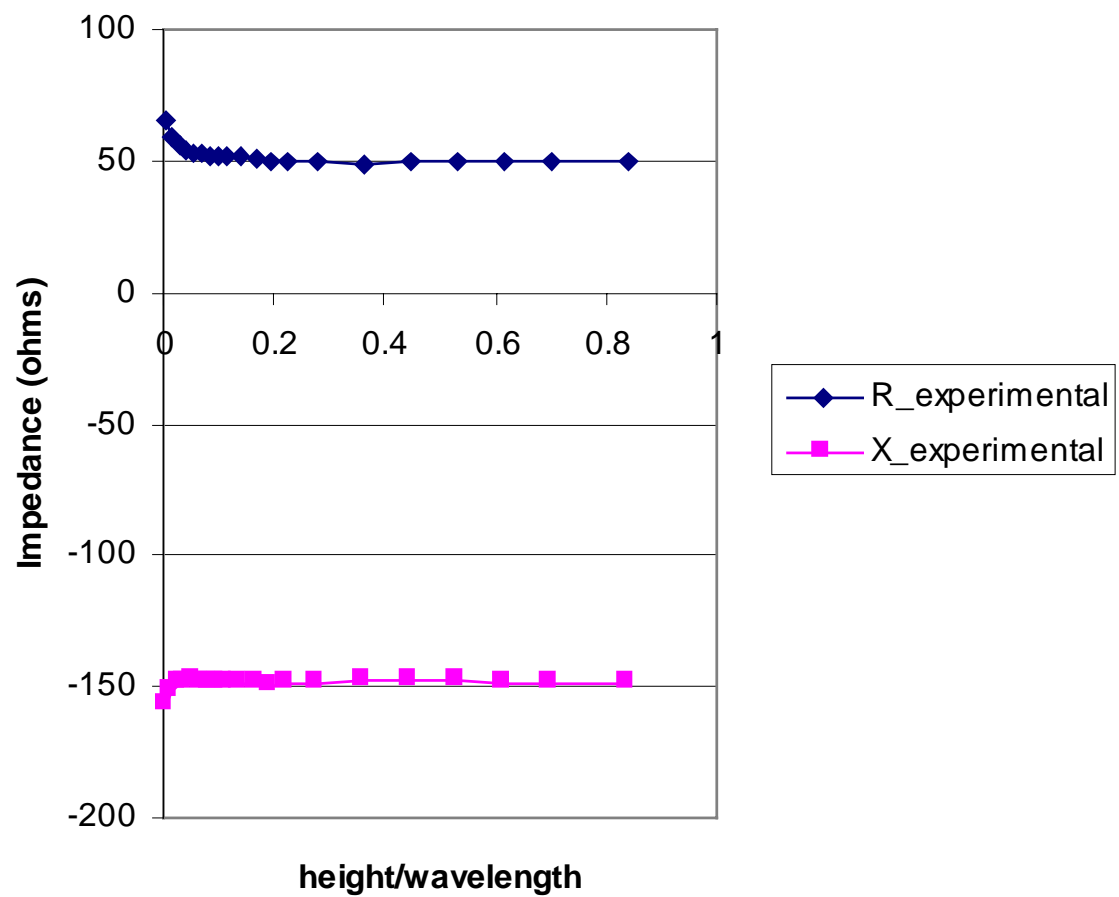

(b) off the $3^{\text {rd }}$ Resonance Frequency when $R \approx 50$ ohms 


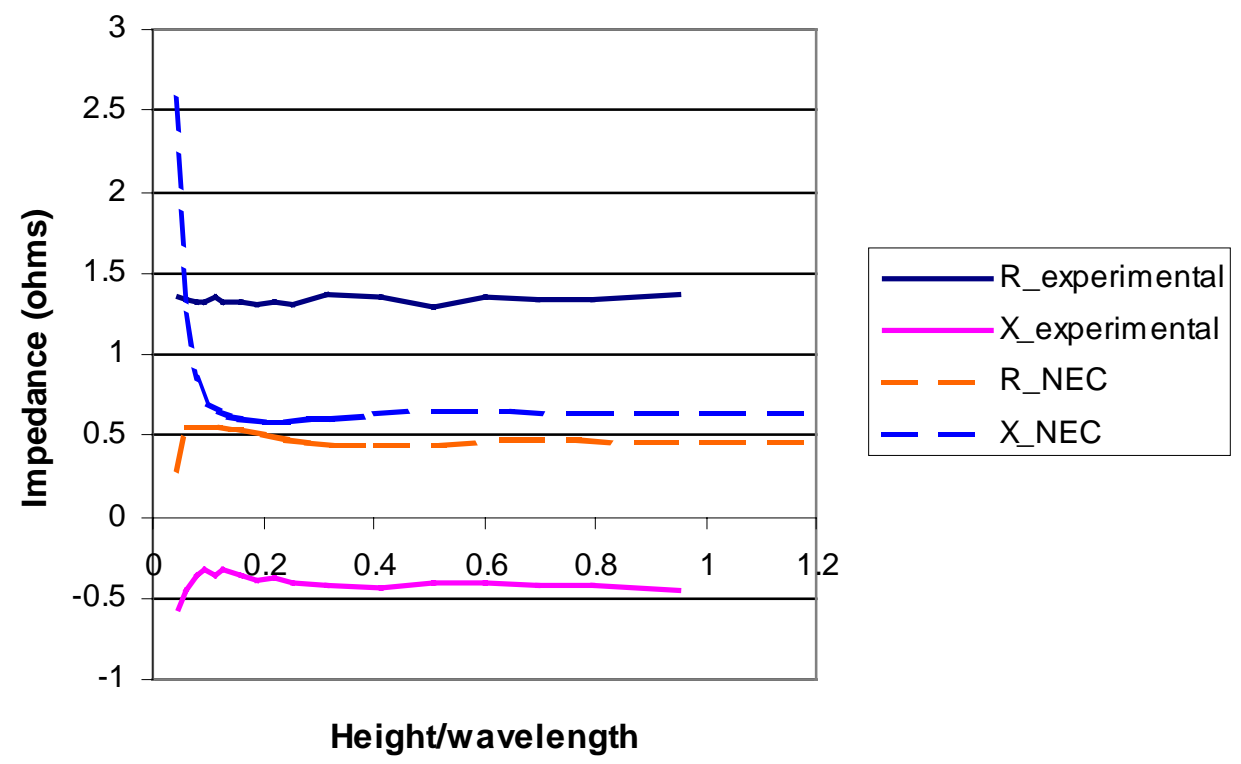

(c) at the $4^{\text {th }}$ Resonance Frequency

Figure 22 Resistance and Reactance of the Horizontal CTHA as a Function of the Relative Height

The experimental results of the CTHA indicate that, similar to the other antennas, the resistance and reactance of the CTHA change as a damped sinusoid function of the distance between the CTHA and the metal plate relative to the free space wavelength. The central axis of each sine wave corresponds to the value of the free space resistance and reactance, respectively.

\section{Summary}

From above comparisons, it can be found on the one hand, there is good agreement between the experimental results and the NEC results for the vertical dipole, the horizontal dipole and the horizontal loop; while on the other hand, an obvious disagreement between the experimental results and the NEC results for the horizontal CTHA. It implies that NEC is a reasonable numerical method for impedance calculation and metallic surface modeling, but the mismodeling of the core material of the CTHA by NEC could result in inaccuracy in calculating the 
CTHA's impedance. To better evaluate the impedance of a CTHA wound on materials with dielectric constant other than one, some other numerical methods need to be used. 


\section{Chapter 5 Applying XFDTD on Impedance Study}

\section{Introduction}

As discussed in Chapter 4, NEC has displayed satisfactory accuracy for calculating the input impedance of the vertical dipole, the horizontal dipole and the horizontal loop. But its results of the impedance of the CTHA disagree with the experimental results. To improve the model of the CTHA, another numerical method, XFDTD, is used.

XFDTD apples the FDTD (the finite difference time domain method) for electromagnetic calculation under the Unix operating system. The method is a transient marching-in-time approach, in which time is divided into small discrete steps and the electric and magnetic fields on a fine grid are calculated at each time step [20]. Being different from NEC, XFDTD allows the user to specify the parameters for dielectric materials, which can be set differently for each grid. This characteristic makes XFDTD being capable of modeling the core material of the CTHA. For this reason, XFDTD is applied to further the study of the impedance change of the CTHA.

\section{Evaluation of XFDTD}

Before XFDTD is applied to study the CTHA, its effectiveness of impedance calculation is evaluated through the study of the vertical dipole over a $3 \mathrm{ft} \times 3 \mathrm{ft}$ metal plate, the same study as mentioned in Chapter 2 and Chapter 3. 
Table 7 Free Space Resonance Frequency and Impedance of the Vertical Dipole

\begin{tabular}{|l|c|c|c|}
\hline & Experiment & NEC & XFDTD \\
\hline Frequency (MHz) & 994.75 & 971 & 1243.9 \\
Resistance (Ohms) & 67.24 & 71.89 & 71.34 \\
Reactance (Ohms) & 0.14 & -0.01 & 0 \\
\hline
\end{tabular}

Table 8 Percentage Difference of the Numerical Results to the Experimental Results

\begin{tabular}{|c|c|c|}
\hline$(\%)$ & NEC & XFDTD \\
\hline Resonance Frequency & 2.4 & 25 \\
Free Space Resistance & 6.9 & 6.1 \\
\hline
\end{tabular}

The relative impedance change as the distance between the vertical dipole and the metal plate changes is plotted in Figure 23. From Figure 23, it can be observed that both the results of NEC and XFDTD agree with the experimental results. All of them exhibit the trend that as the distance between the vertical dipole and the metal plate increases, the relative impedance change decreases along a spiral curve, which ends at the origin of the coordinate system when the distance is larger than $100 \mathrm{~mm}$. It indicates that XFDTD provides a reliable numerical method for impedance calculation. 


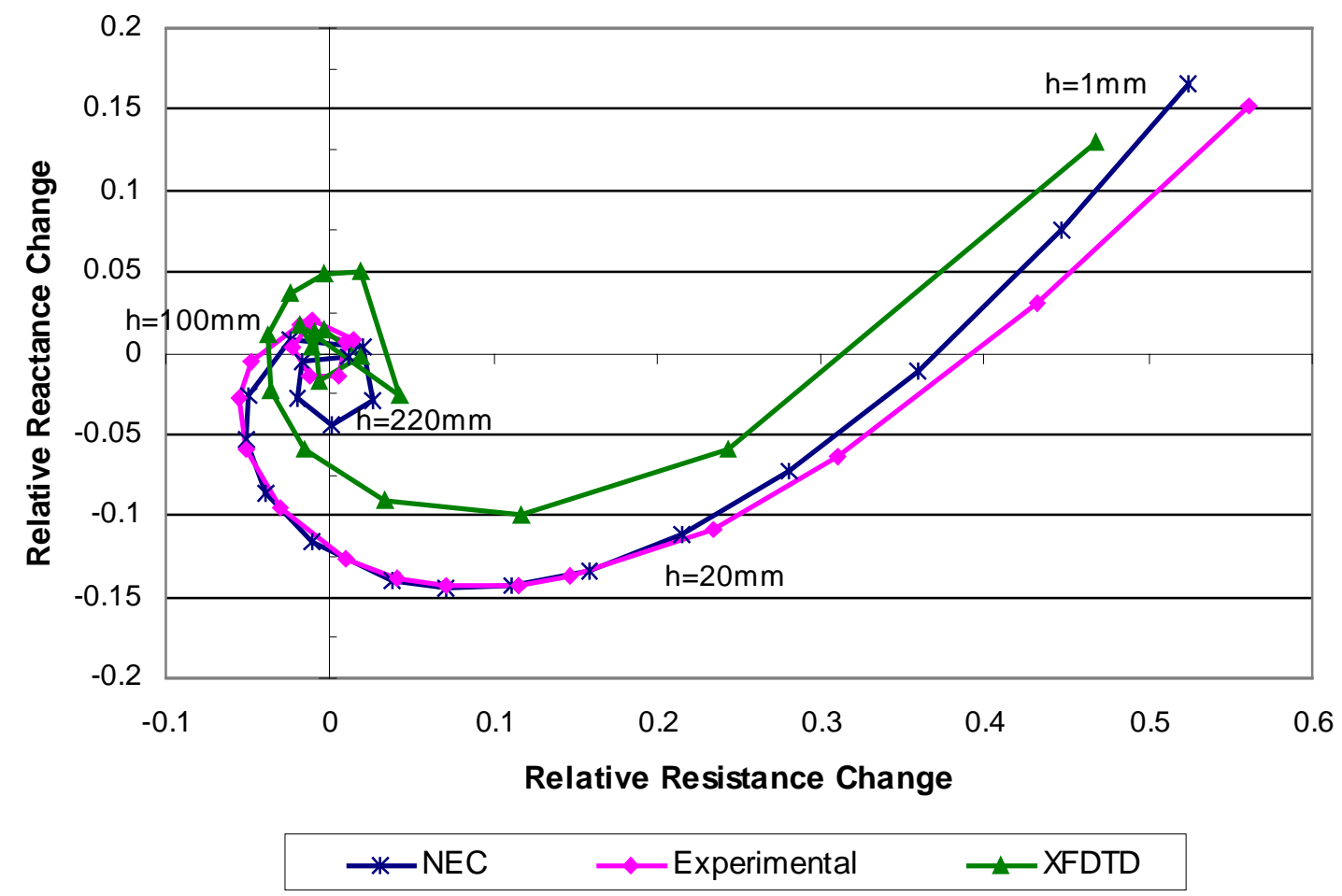

Figure 23 Relative Impedance Change of the Vertical Dipole over a 3'x3' Metal Plate

\section{Applying XFDTD on the Metal Plate Study of the CTHA}

Using XFDTD, it needs to discretize the whole space involved in the metal plate study. To maintain the accuracy as well as to save some time for the calculation, the $3 \mathrm{ft} x 3 \mathrm{ft}$ plate is replaced by a 4 in $x$ in plate.

The core material of the CTHA is modeled by XFDTD with the dielectric constant of 1.75 . For this model, the free space resonance frequencies of the CTHA predicted by XFDTD agree well with the experimental results.

Table 9 lists the free space resonance frequencies and impedance of the CTHA resulted from experiment, NEC and XFDTD. Table 10 lists the percentage difference of the NEC results and the XFDTD results comparing to the experimental results. 
Table 9 Free Space Resonance Frequencies and Impedance of the CTHA

\begin{tabular}{|l|l|l|l|l|l|l|l|l|l|}
\hline & \multicolumn{3}{|c|}{$3^{\text {rd }}$ Resonance } & \multicolumn{3}{c|}{ R $\approx 50$ ohm } & \multicolumn{3}{c|}{$4^{\text {th }}$ Resonance } \\
\cline { 2 - 10 } & Exp. & NEC & XFDTD & Exp. & NEC & XFDTD & Exp. & NEC & XFDTD \\
\hline Freq. & 739 & 917 & 727 & 841 & 938 & 835 & 952 & 1215 & 949 \\
R & 575 & 35843 & 17 & 50 & 51 & 65 & 1.4 & 0.5 & 676 \\
\hline
\end{tabular}

Table 10 Percentage Difference of the Numerical Results to the Experimental Results

\begin{tabular}{|c|c|c|c|c|c|c|}
\hline \multirow{2}{*}{$(\%)$} & \multicolumn{2}{|c|}{$3^{\text {rd }}$ Resonance } & \multicolumn{2}{c|}{ R $\approx 50$ ohms } & \multicolumn{2}{c|}{$4^{\text {th }}$ Resonance } \\
\cline { 2 - 7 } & NEC & XFDTD & NEC & XFDTD & NEC & XFDTD \\
\hline Frequency & 24.1 & 1.6 & 11.5 & 0.7 & 27.6 & 0.5 \\
Resistance & 6100 & 97 & $* *$ & $* *$ & 66 & 48200 \\
Reactance & $*$ & $*$ & 810 & 249 & $*$ & $*$ \\
\hline
\end{tabular}

* for the resonance frequencies, taking the reactance to be zero

** for $\mathrm{R} \approx 50 \mathrm{ohms}$, taking the resistance to be $50 \mathrm{ohms}$

From Table 10, it can be found that XFDTD, which has taken into account the core material of the CTHA, has provided more accurate prediction than NEC on the free space resonance frequencies and impedance for the $3^{\text {rd }}$ resonance and off the $3^{\text {rd }}$ resonance when $\mathrm{R} \approx 50 \mathrm{ohms}$.

Figure 24 illustrates the relative impedance change of the CTHA over the 4" x 4" metal plate resulted from the experiment, NEC and XFDTD. On the plots, $h_{r}$ represents the distance relative to the free space wavelength. In each of the three cases, namely at the $3^{\text {rd }}$ resonance frequency, off the $3^{\text {rd }}$ resonance frequency when $R \approx 50 \mathrm{ohms}$, and at the $4^{\text {th }}$ resonance frequency, the relative 
impedance change of the CTHA decreases as the distance increases at a similar trend for all the three results. But the gradients of the decrease for the three results are different. The NEC results display a relatively slower decrease comparing with the experimental results, and the XFDTD results display a relatively faster decrease. For a better view of the difference, the magnitude of the relative impedance change of the CTHA is calculated:

$$
\text { Magnitude }=\sqrt{\frac{\left(R-R_{0}\right)^{2}+\left(X-X_{0}\right)^{2}}{R_{0}{ }^{2}}}
$$

The magnitude of the relative impedance change corresponds to the distance from each point on the relative impedance change curve to the origin. Figure 25 illustrates the magnitude of the relative impedance change of the CTHA as a function of the relative distance. It can be found the XFDTD results agree well with the experimental results.

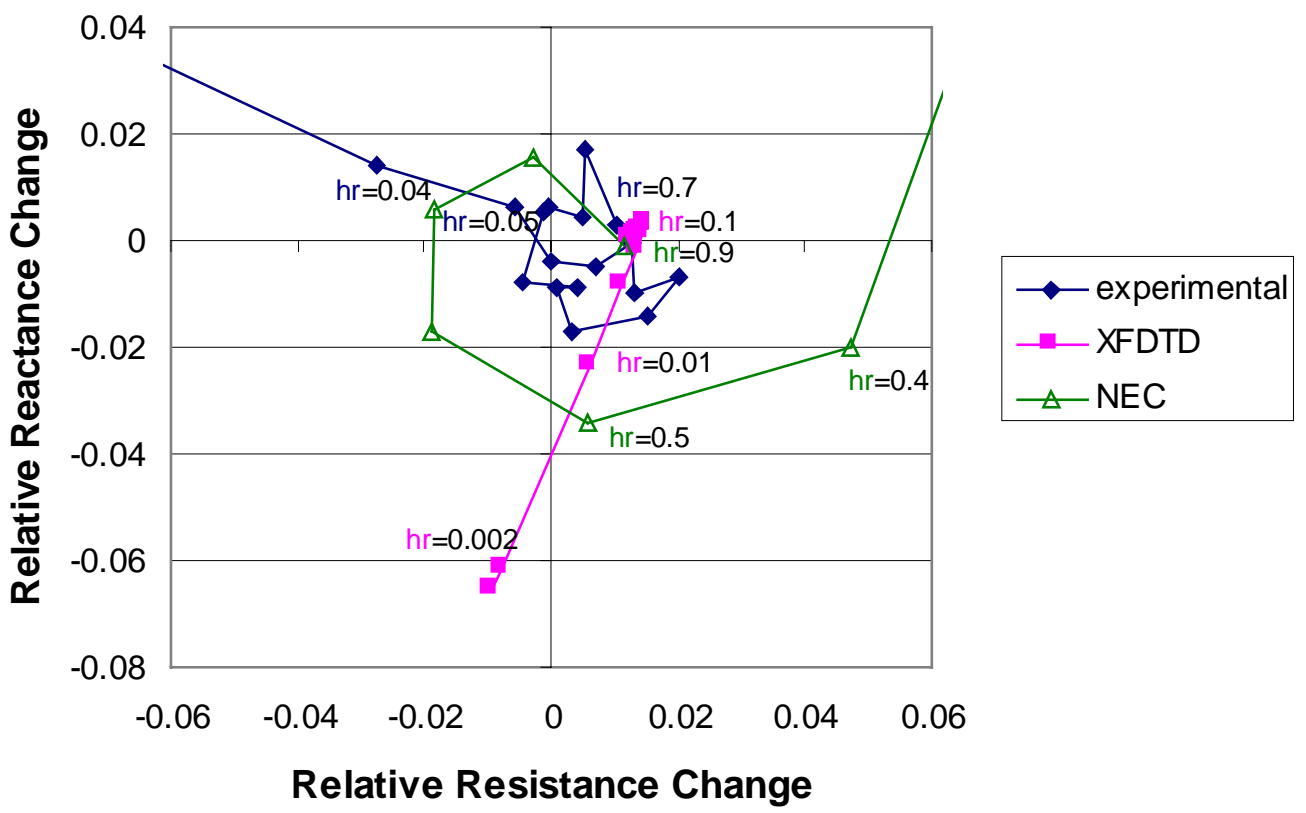

(a) at the $3^{\text {rd }}$ Resonance Frequency 


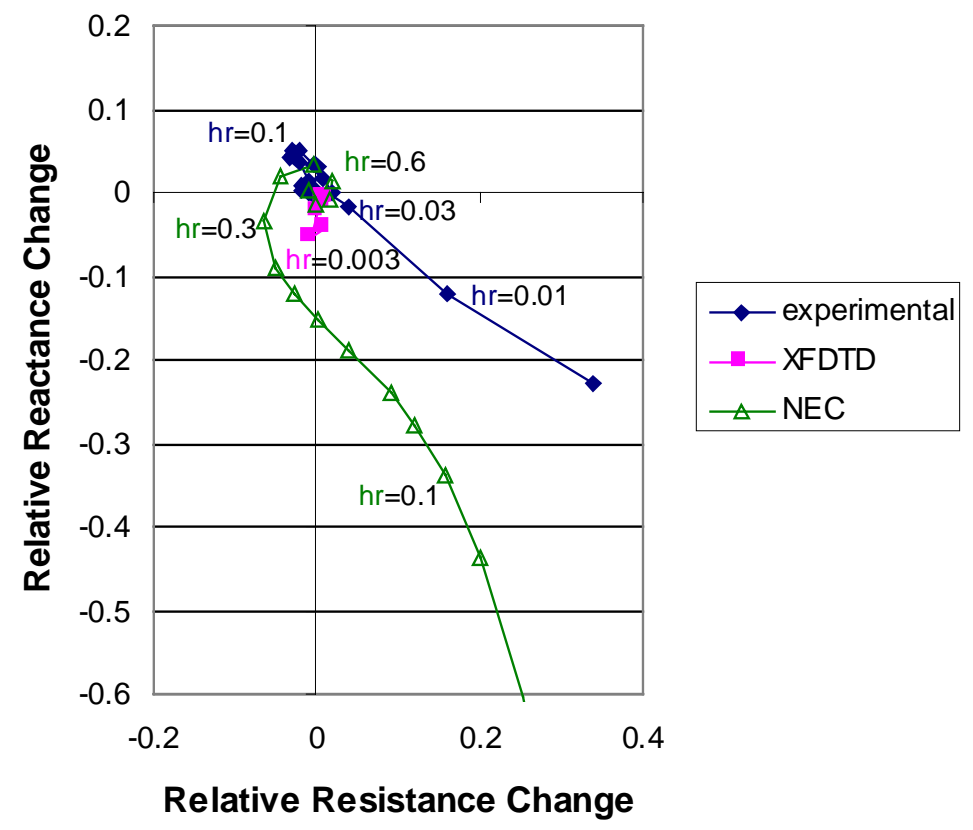

(b) off the $3^{\text {rd }}$ Resonance Frequency when $R \approx 50 \mathrm{ohm}$

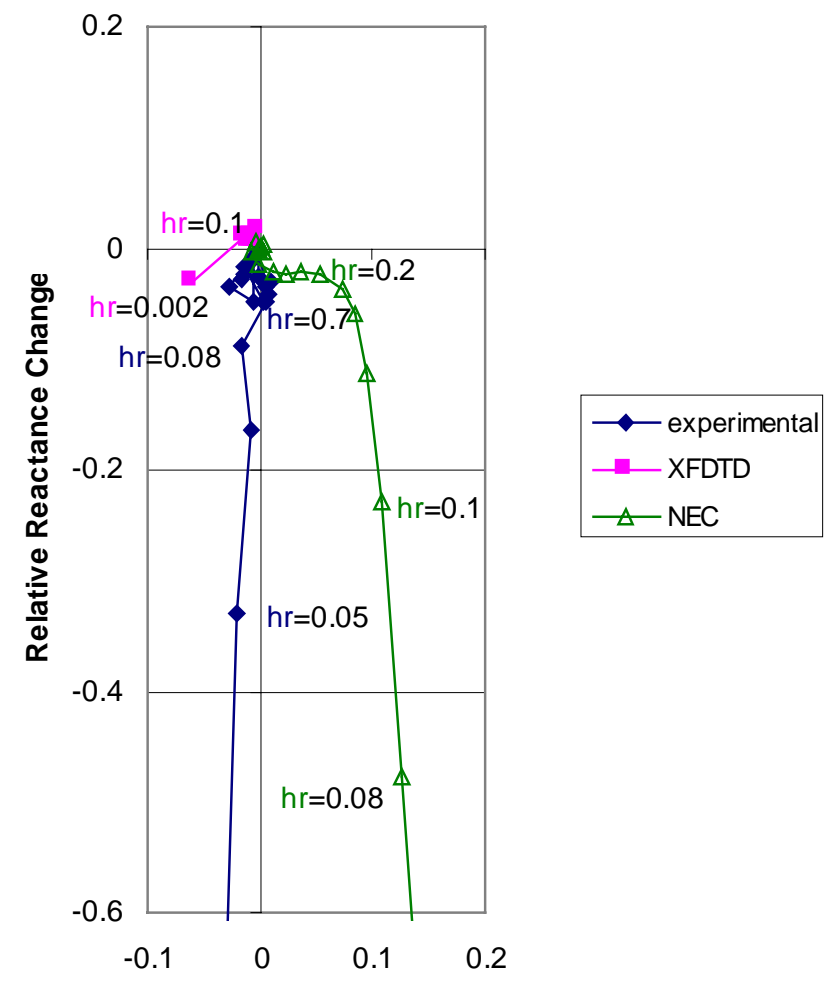

Relative Resistance Change

(c) at the $4^{\text {th }}$ Resonance Frequency

Figure 24 Relative Impedance Change of the CTHA over a 4"x4" Metal Plate 


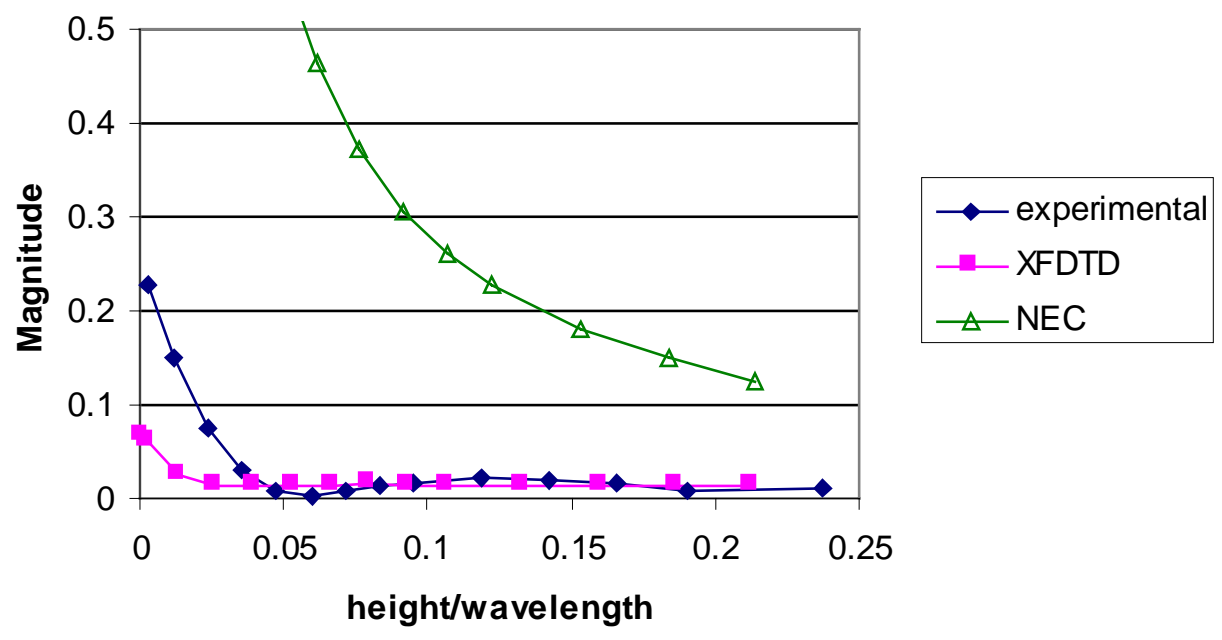

(a) at the $3^{\text {rd }}$ Resonance Frequency

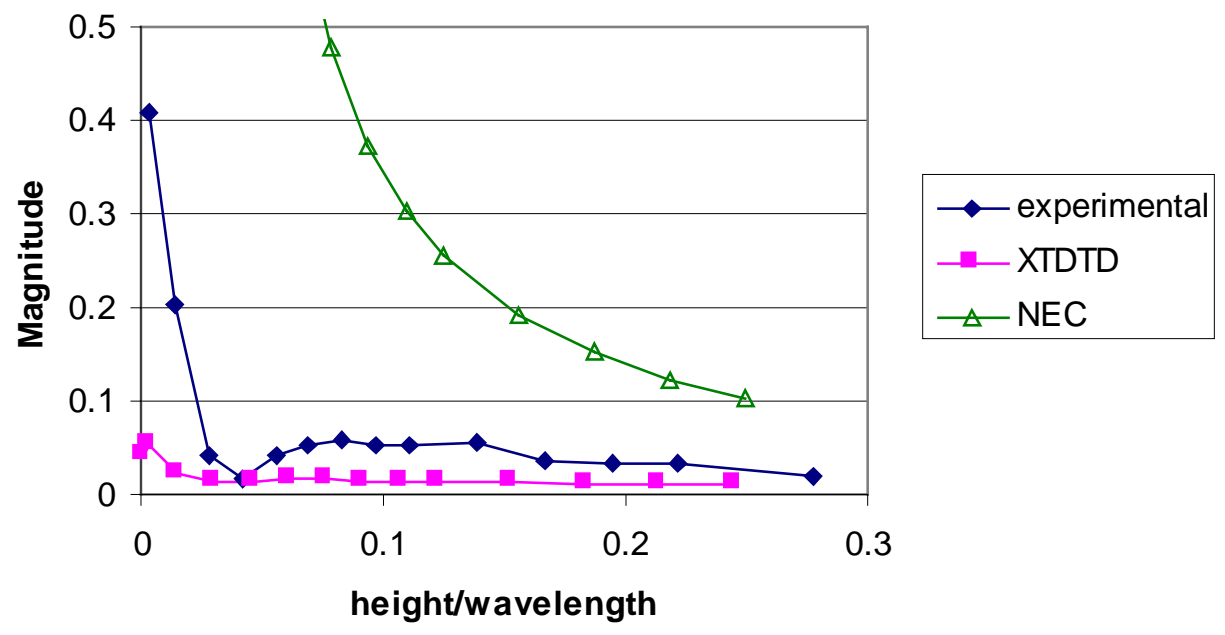

(b) off the $3^{\text {rd }}$ Resonance Frequency when $R \approx 50 \mathrm{ohm}$ 


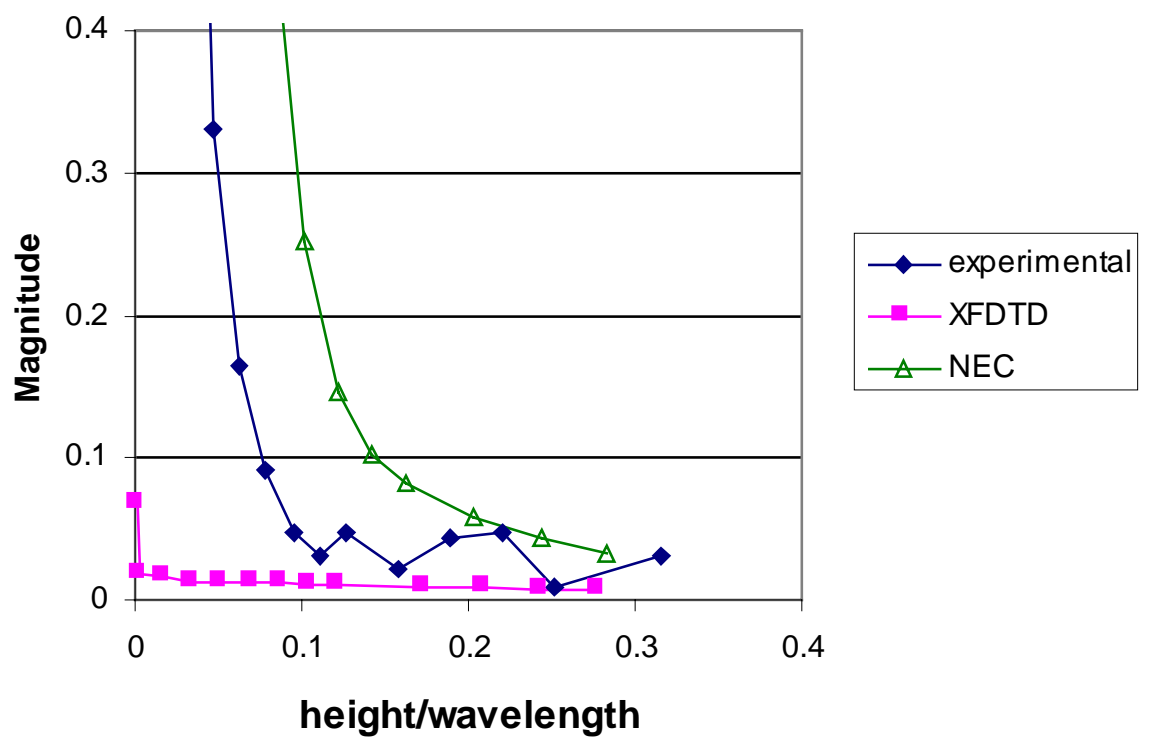

(c) at the $4^{\text {th }}$ Resonance Frequency

Figure 25 Magnitude of the Relative Impedance Change of the CTHA over a 4"x4" Metal Plate

\section{Summary}

The agreement between the XFDTD and the experimental results indicates that XFDTD is a reliable numerical method for impedance study. It is a better choice comparing to NEC for modeling the CTHAs. But the calculation of each point on the relative impedance Change curve with XFDTD takes much more time (several hours per point) than with NEC (several minutes per point). 


\section{Chapter 6 Comparison of Antennas}

\section{Introduction}

In Chapter 4, the numerical and experimental results for the relative impedance change of antennas over a $3 \mathrm{ft} \times 3 \mathrm{ft}$ metal plate were presented and compared. From the relative impedance change plots, it can be observed that different antennas have differently shaped spirals. The magnitude of the relative impedance change, which is defined as Eq.6-1, represents the size of the spiral curves. For comparing the impedance effect of the metal plate on each antenna, the magnitudes of the relative impedance change of antennas are compared, and the relationship between the magnitude of the relative impedance change and the antenna's SWR (standing wave ratio) is analyzed.

In addition, the reason why the CTHA is relatively invariant with its impedance is explored by comparing the impedance effect of the metal plate on the CTHA and a single helix torioid antenna, and by comparing the near field energy distribution under the horizontal dipole and under the CTHA.

\section{Magnitude of the Relative Impedance Change}

The magnitude of the relative impedance change is defined for comparing the impedance effect of the metal plate on different antennas by

$$
\text { Magnitude }=\sqrt{\left(\frac{\Delta R}{R_{0}}\right)^{2}+\left(\frac{\Delta X}{R_{0}}\right)^{2}}
$$

where $\Delta R=R-R_{0}, \Delta X=X-X_{0}$. 
Eq.6-1 gives the definition of the magnitude of the relative impedance change. The smaller the magnitude, the less variant the antenna's impedance as compared to free space impedance. Figure 26 shows the magnitude of each antenna's results from the experiment as a function of the relative height to the free space wavelength. It is clear from Figure 26 that CTHA at its $3^{\text {rd }}$ resonance frequency and off resonance frequency has the least variance of its relative impedance change. At the $4^{\text {th }}$ resonance frequency, its relative impedance change is larger than that of the vertical dipole, but smaller than those of the horizontal dipole and horizontal loop.

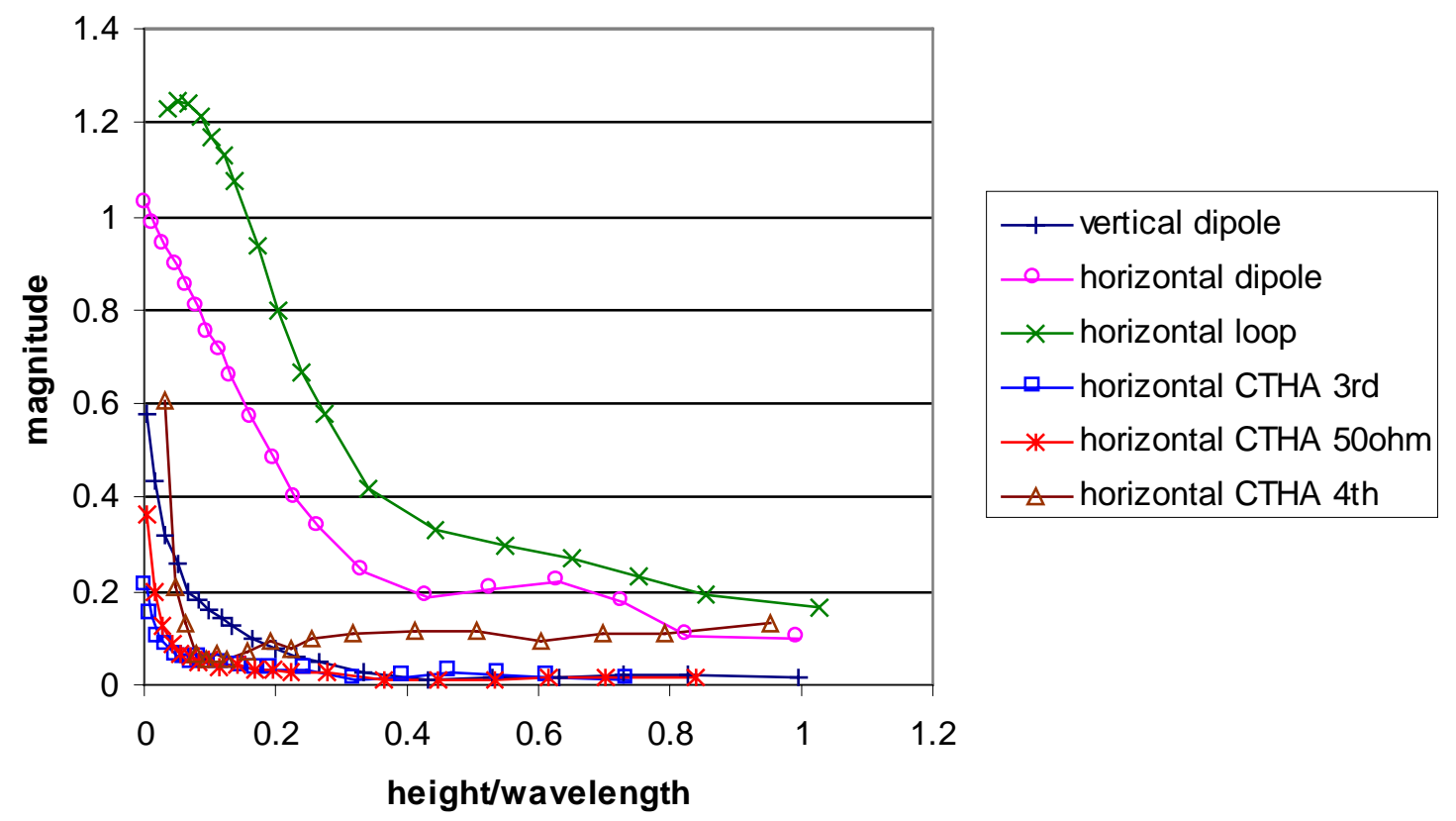

Figure 26 Magnitude of the relative Impedance Change Resulted from the Experimental Results

\section{Effect of the Relative Impedance Change on SWR}

According to the definition in [21], the voltage reflection coefficient is the ratio of the reflected voltage at a given point on a transmission line to the incident voltage. The reflection coefficient is determined by the relationship between the characteristic impedance of the transmission line and the actual load at the terminated end of the line. For high-quality, low-loss 
transmission lines, the characteristic impedance $\mathrm{Z}_{\mathrm{c}}$ is almost completely resistive, meaning that $\mathrm{Z}_{\mathrm{c}} \approx \mathrm{R}_{\mathrm{c}}$ and $\mathrm{X}_{\mathrm{c}} \approx 0$. Thus the magnitude of reflection coefficient could be expressed as:

$$
|\rho|=\sqrt{\frac{\left(R_{a}-R_{c}\right)^{2}+X_{a}{ }^{2}}{\left(R_{a}+R_{c}\right)^{2}+X_{a}^{2}}},
$$

For an ideally matched antenna:

$$
R_{a}=R_{c}
$$

and

$$
X_{a}=0,
$$

such that $|\rho|=0$.

Assuming that the existence of an object in the vicinity of the antenna causes a change of resistance $\Delta \mathrm{R}$ and reactance $\Delta \mathrm{X}$ of the antenna, the magnitude of the reflection coefficient will be:

$$
\left|\rho^{\prime}\right|=\sqrt{\frac{\left(R_{a}+\Delta R-R_{c}\right)^{2}+\left(X_{a}+\Delta X\right)^{2}}{\left(R_{a}+\Delta R+R_{c}\right)^{2}+\left(X_{a}+\Delta X\right)^{2}}} .
$$

Substituting Eq.6-3 and Eq.6-4 into Eq.6-5, the magnitude of changed reflection coefficient can be simplified to Eq.6-6. Dividing $\mathrm{R}_{0}$, the free space resistance of the antenna, by both the numerator and denominator of $\left|\rho^{\prime}\right|$, Eq. 6-6 results in Eq.6-7.

$$
\begin{aligned}
& \left|\rho^{\prime}\right|=\sqrt{\frac{(\Delta R)^{2}+(\Delta X)^{2}}{\left(\Delta R+2 R_{c}\right)^{2}+(\Delta X)^{2}}} . \\
& \left|\rho^{\prime}\right|=\sqrt{\frac{(\Delta R)^{2} / R_{0}^{2}+(\Delta X)^{2} / R_{0}{ }^{2}}{\left(\Delta R+2 R_{c}\right)^{2} / R_{0}{ }^{2}+(\Delta X)^{2} / R_{0}{ }^{2}}} .
\end{aligned}
$$


According to the definition of the magnitude of the relative impedance change (Eq.6-1), the relationship between the magnitude of the reflection coefficient and the magnitude of the relative impedance change (abbreviated as $\mathrm{m}$ ) can be expressed as Eq.6-8. Because $\Delta \mathrm{R}$ is much smaller than $R_{c}$, which is a constant for all antennas, the reflection coefficient will increase as the magnitude of the relative impedance change increases.

$$
\left|\rho^{\prime}\right|=\sqrt{\frac{1}{1+4\left(\Delta R R_{c}+R_{c}^{2}\right) / m^{2}}} .
$$

The SWR is related to the magnitude of the complex reflection coefficient by Eq.6-9. Because $|\rho|$ is larger than 0 and less than 1, Eq. 6-9 indicates that the increase of the magnitude of the reflection coefficient will raise the value of the SWR. According to the positive relation between the magnitude of the relative impedance change and the magnitude of the reflection coefficient, as well as the positive relation between the magnitude of the reflection coefficient and the SWR, it can be concluded that larger magnitude of the relative impedance change would result in larger increase of the SWR.

$$
S W R=\frac{1+|\rho|}{1-|\rho|}
$$

From the above discussion, CTHA has relatively smaller magnitude of the relative impedance change when compared to the horizontal dipole and horizontal loop. This means in cases where there is an object in the vicinity of the antenna, CTHA will have a smaller increase of SWR than will the other antennas. 


\section{Change of the Resonance Frequency}

The metallic ground plane in the vicinity of an antenna will change the antenna's impedance. It will also change the antenna's resonance frequency. Based on the experimental results, the resonance frequency of the antennas at each height above the $3 \mathrm{ftx} 3 \mathrm{ft}$ metal plate reference to the free space resonance frequency is plotted as a function of the height relative to the free space wavelength in Figure 27.

From Figure 27, it can be observed that for all the antennas studied, the resonance frequency reference to the free space resonance frequency oscillates around the value of 1 . As the height of the antenna above the metal plate increases, the amplitude of the oscillation decreases. It is expected that as the height tends to be infinity, f/f0 tends to be 1, which means when the metal plate is very far away, the resonance frequency will be equal to the free space resonance frequency.

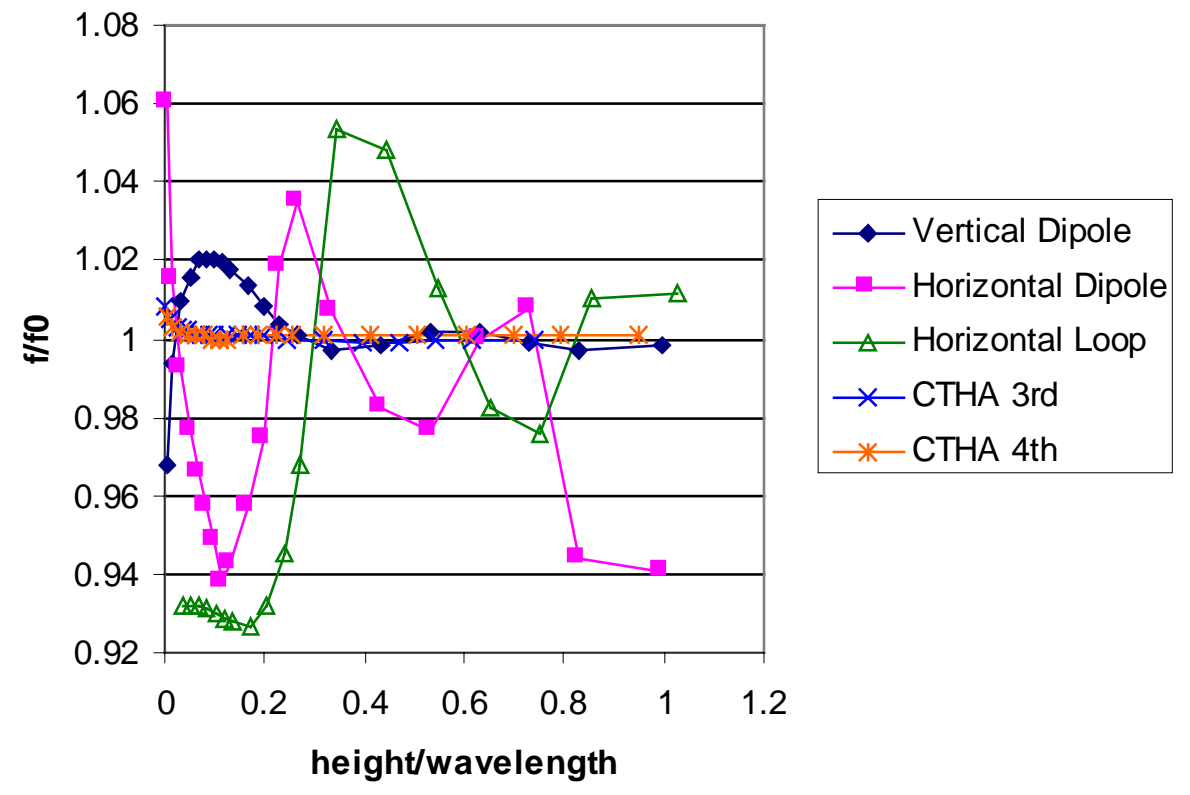

Figure 27 Resonance Frequency Reference to the Free Space Resonance Frequency 
Comparing the resonance frequency change of the antennas due to the metal plate in the vicinity, it can be found that the horizontal CTHA has the least change at both the $3^{\text {rd }}$ resonance and the $4^{\text {th }}$ resonance. This agrees with the conclusion mentioned above that the CTHA's input impedance is the least variant due to the metal plate in the vicinity. In other words, the metal plate has the least effect on the horizontal CTHA.

\section{Exploration of the Reasons for CTHA's Relative Impedance Invariance}

Above discussions indicate that CTHA is relatively impedance invariant to the metallic ground plane in the vicinity. Then comes the question why the CTHA has the characteristic of being relative impedance invariant. The reasons have been explored through studying its physical structure and the characteristic of its near field distribution.

\section{1) Effect of Wire Coupling}

Considering that the CTHA is a contrawound toroidal helical antenna, the coupling of the two single helices may be an explanation for CTHA's relative impedance invariance. To examine this possibility, the impedance effect of a metal plate on a single helix toroid antenna has been compared with CTHA by numerical modeling with NEC. The single helical toroid antenna has been modeled with the same dimensions as CTHA.

Figure 28 and Figure 29 graph the relative impedance change and the magnitude of the relative impedance change for the single helical toroid antenna and the CTHA. A comparison of the two shows that the coupling of the two single helices of the CTHA has not significantly changed the spiral shape of the relative impedance change curve, but the size of the spirals at different frequencies has decreased. From Figure 29, it can be more clearly observed that the 
magnitude of the relative impedance change of the CTHA is much smaller than that of the single helical toroid antenna.

By modeling with NEC, the effect of the core material of the CTHA has been taken out of consideration. The difference of the impedance effect of the metal plate on the CTHA comparing with that on the single helix toroid antenna can be explained by the difference of the structure of the two antennas. Consequently, it can be concluded that the coupling of the two helices of the CTHA helps to explain the relative impedance invariance of the CTHA.

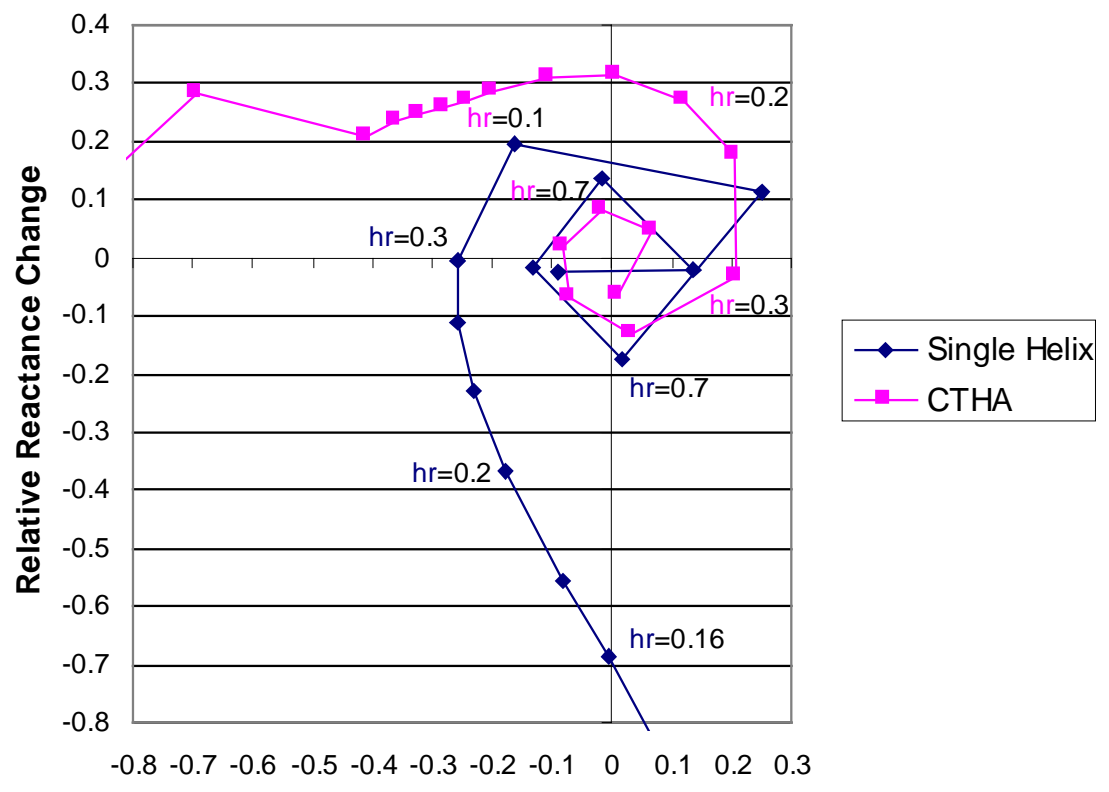

Relative Resistance Change

(a) at $3^{\text {rd }}$ Resonance Frequency 


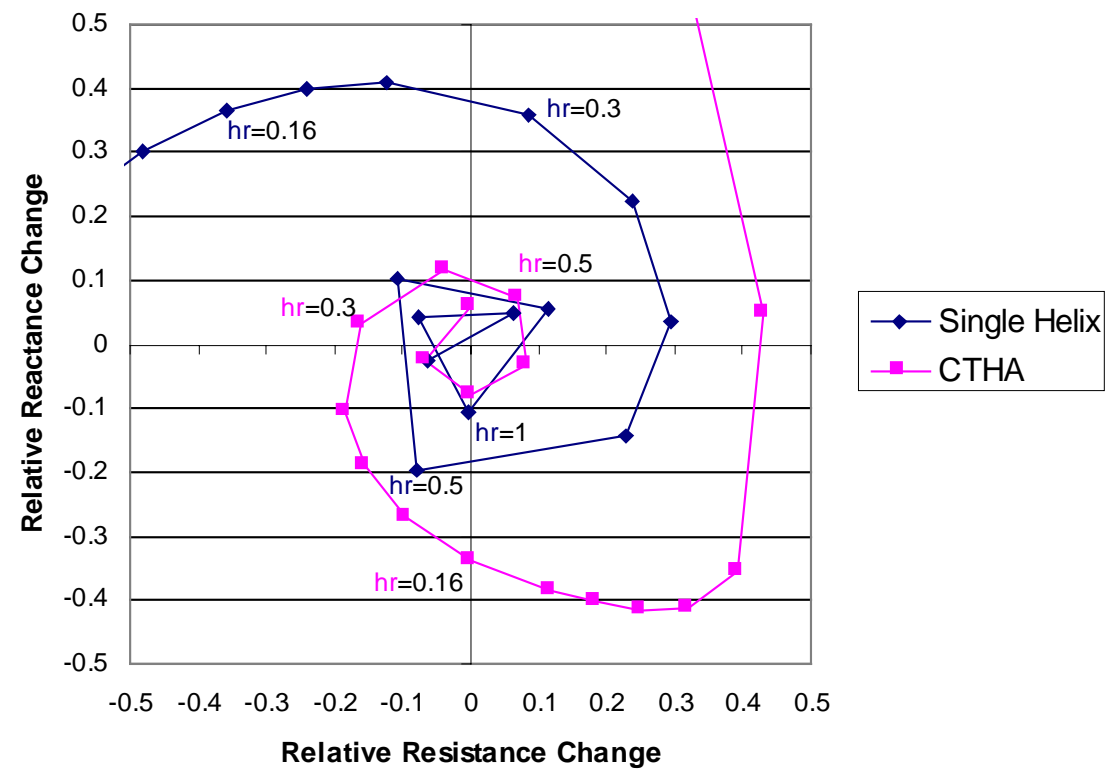

(b) off $3^{\text {rd }}$ Resonance Frequency when $R \approx 500 h m s$

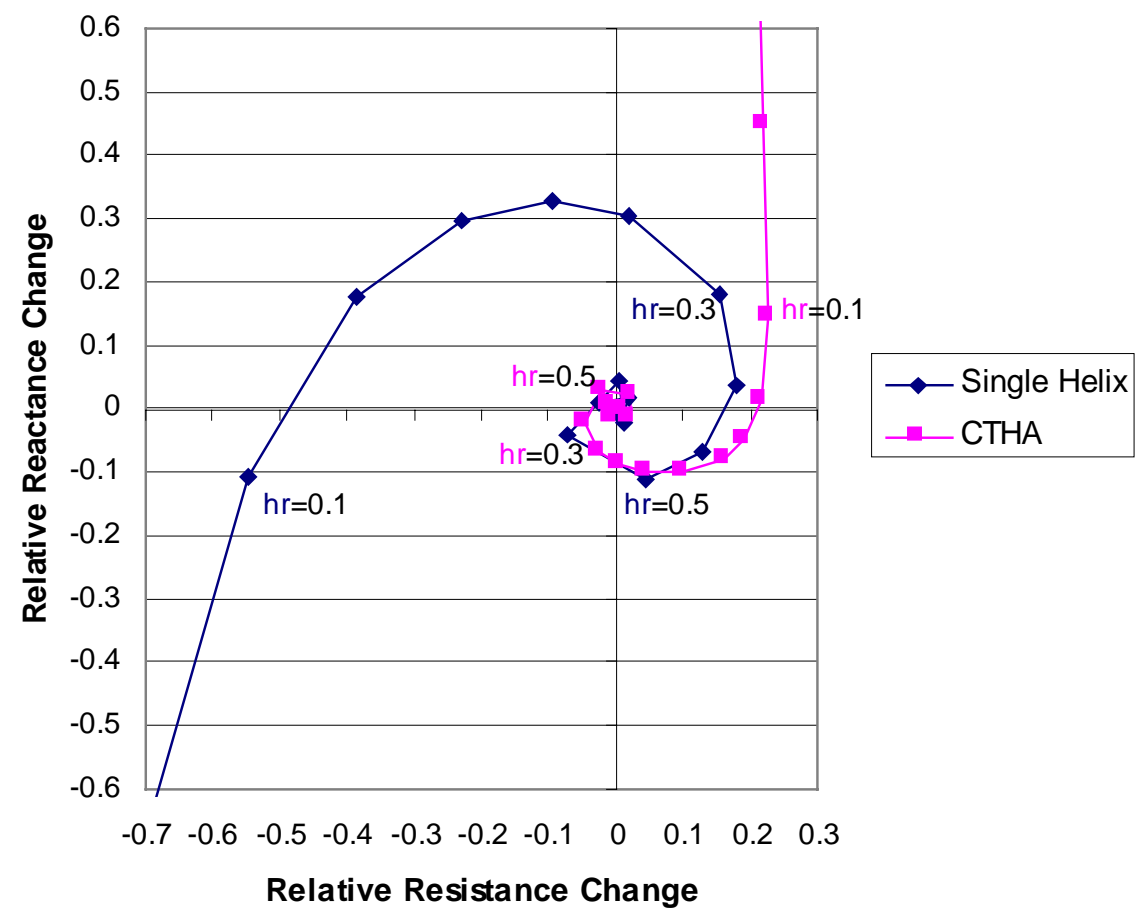

(c) at $4^{\text {th }}$ Resonance Frequency

Figure 28 Relative Impedance Change of the Single Helix and the CTHA Resulted from NEC 


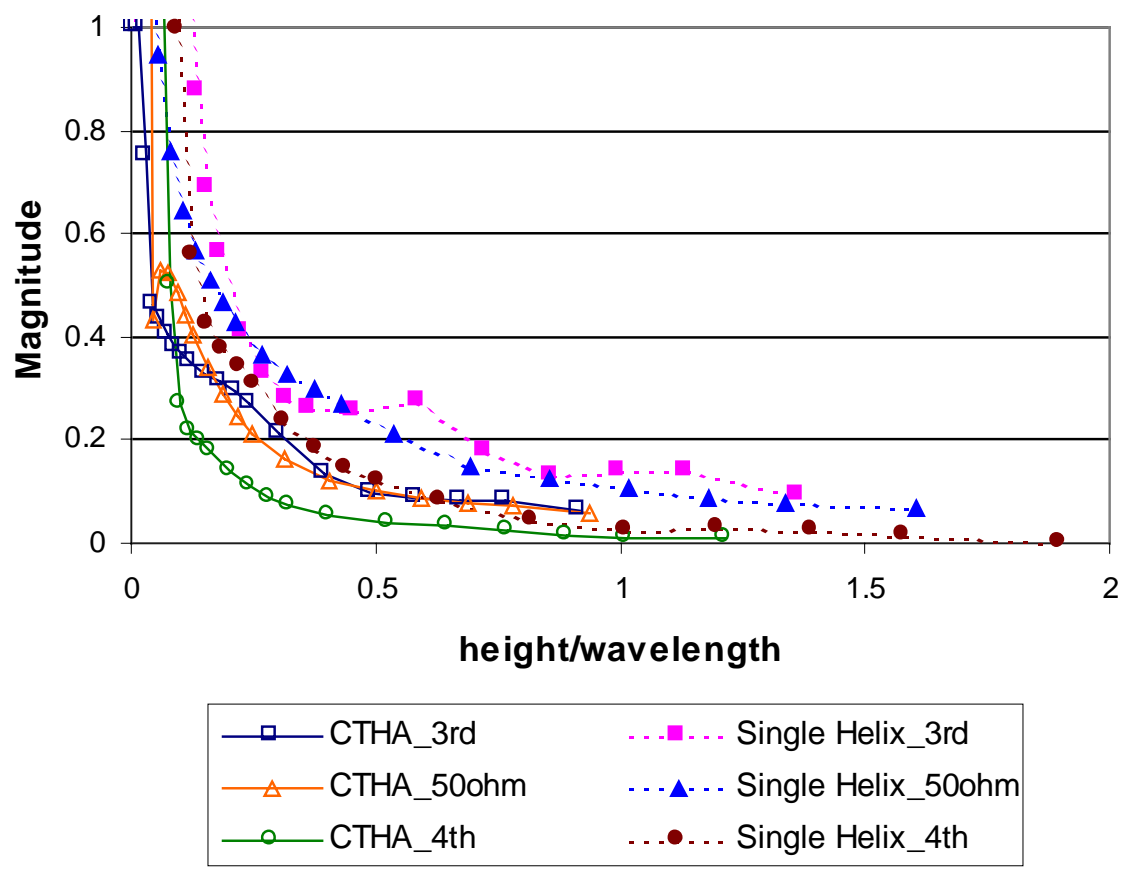

Figure 29 Magnitude of the relative Impedance Change of the Single Helix and the CTHA Resulted from NEC

\section{2) Effect of Near Field Energy Distribution}

Considering that significant impedance variance occurs when the distance between an antenna and a metallic ground plane is less than one wavelength, it is necessary to study the antenna's near field pattern to find more explanation for CTHA's relative impedance invariance.

The near electric and magnetic fields of the horizontal dipole and the CTHA (at $3^{\text {rd }}$ resonance frequency) are evaluated numerically from NEC results. Figure 30 illustrates the electric field intensity $(\mathrm{E})$ and magnetic field intensity $(\mathrm{H})$ within the volume under the horizontal dipole. The width and height of the volume are about the same as the length of the dipole, and the thickness is two times the diameter of the dipole. The upper surface of the volume corresponds to the cross section of the horizontal dipole. 


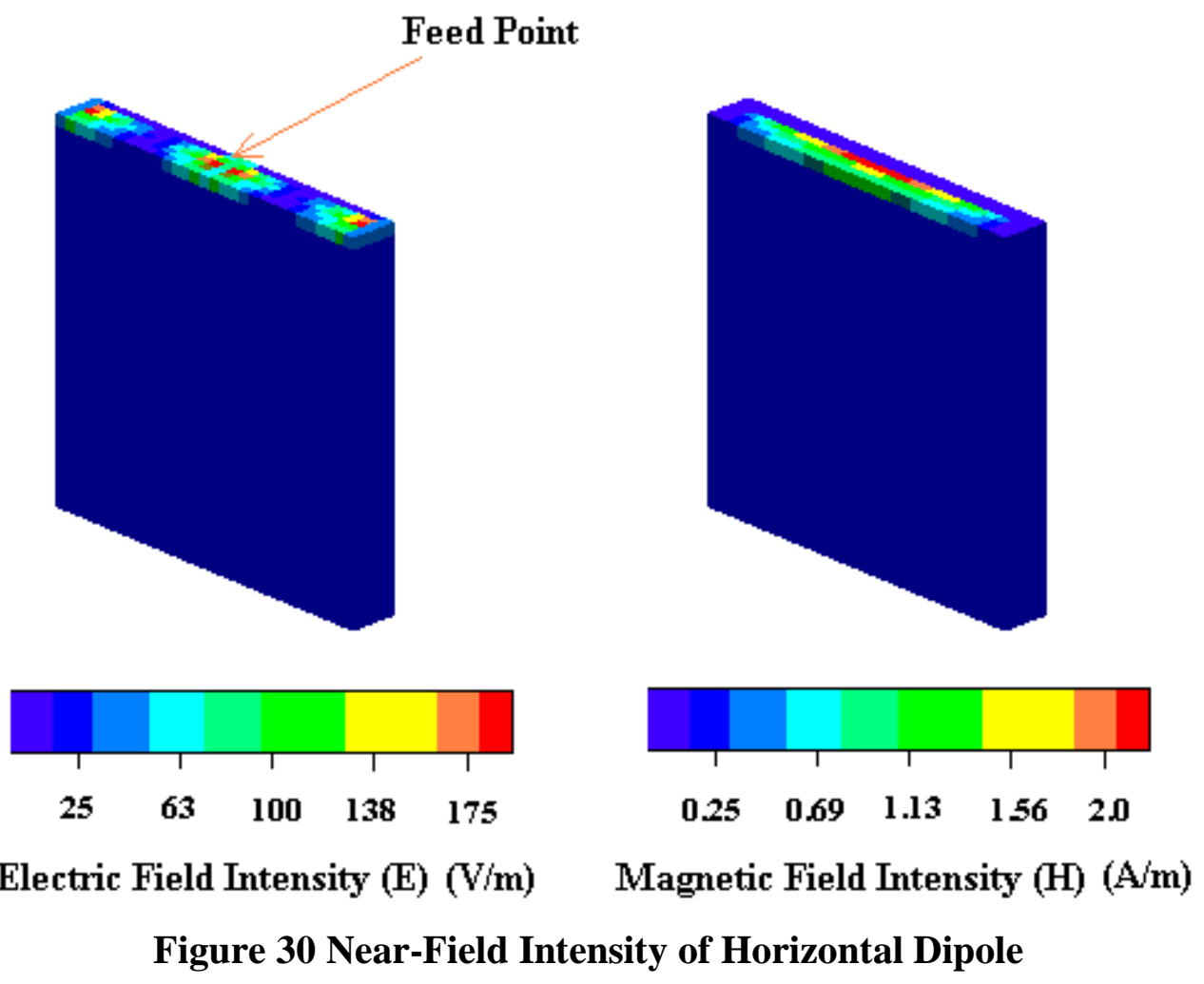

Figure 31 illustrates the electric field intensity and magnetic field intensity within the volume under the CTHA. The width, height, and thickness are about the same as the outer diameter of the CTHA. The upper surface of the volume corresponds to the cross section of the CTHA. 


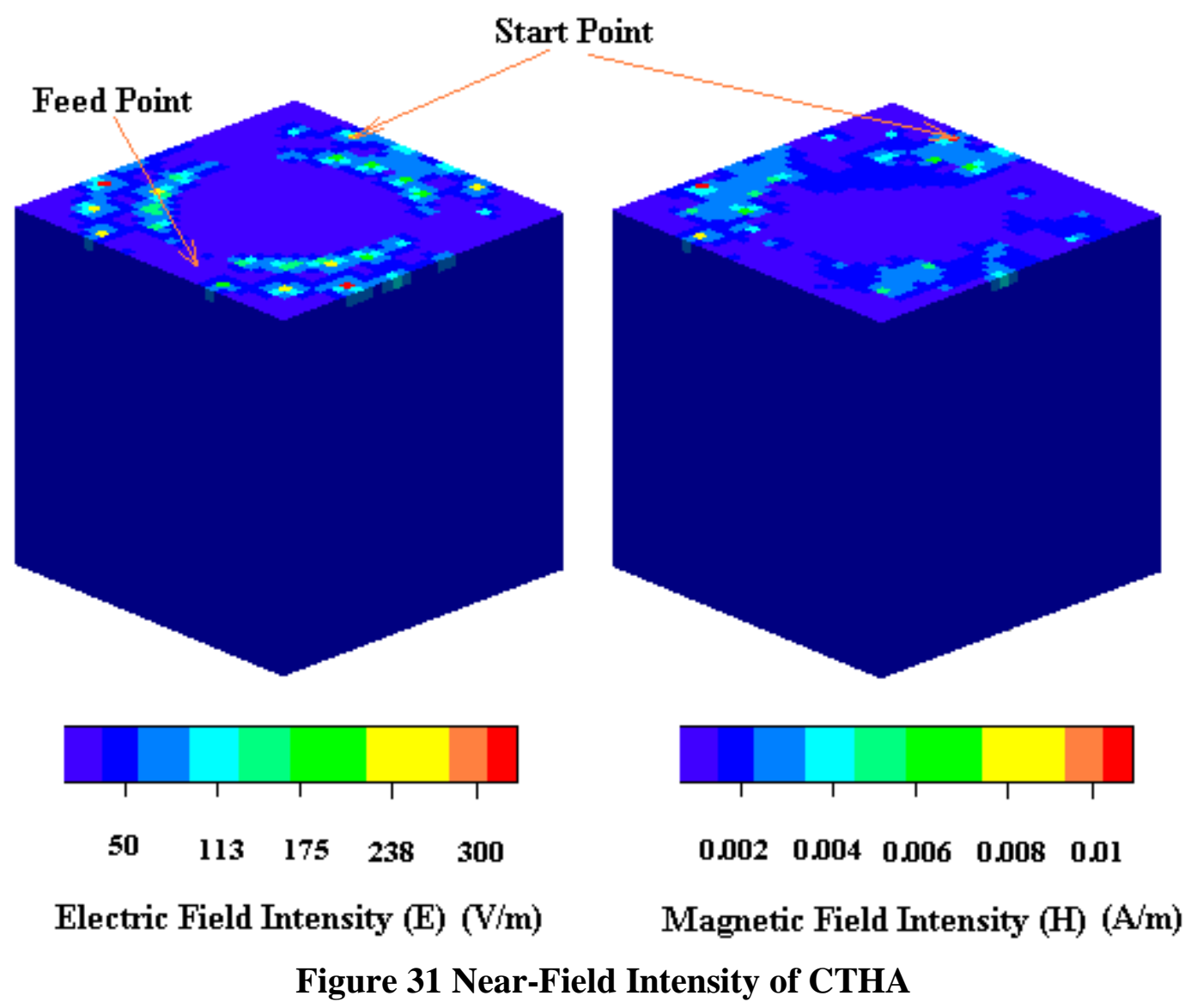

From the above field intensity contours, it can be observed that the near field energy has concentrated around the feed point and two ends of the horizontal dipole, while relatively even distributed around the CTHA. Because the field intensity has decreased sharply along the height, the vertical distribution of the near field energy can not be illustrated clearly by the 3dimensional illustration. To get a better view of the vertical distribution of the near-filed energy, the electric flux density and magnetic flux density are calculated according to Eqs. 6-10 and 6-11 along a vertical line for each antenna. The starting point of the line is chosen with the maximum value on the upper surface of the above 3-D illustrations. For the horizontal dipole, the starting point is next to the feed point along the axis of the dipole, and for the CTHA, it is opposite the feed point as indicated in Figure 31. 


$$
\begin{aligned}
& D=\varepsilon_{0} E \\
& B=\mu_{0} H
\end{aligned}
$$

Figure 32 compares the electric flux density along the vertical lines under the horizontal dipole and the CTHA. It indicates that CTHA has its near electric field energy much more concentrated within $0.1 \lambda$ (about $33 \mathrm{~mm}$ ) beneath the center of it, while the horizontal dipole has relatively larger near electric field energy distributed along the vertical line until the height is close to $1 \lambda$.

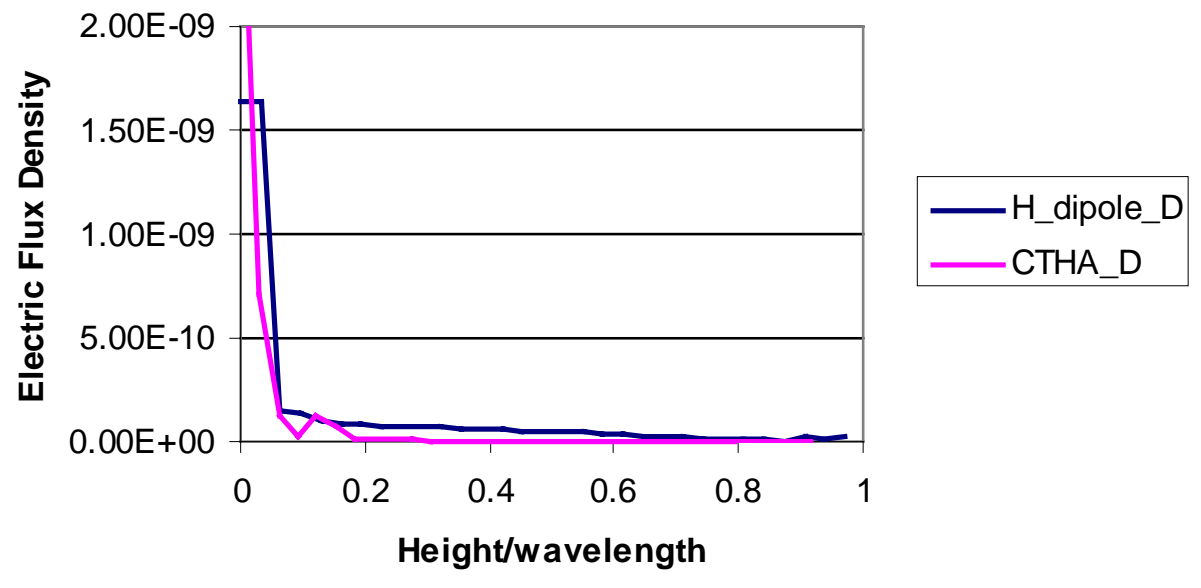

Figure 32 Comparison of Electric Flux Density

Figure 33 compares the magnetic flux density along the vertical lines under the two antennas. Obviously, the horizontal dipole has much larger near magnetic field energy than the CTHA. 


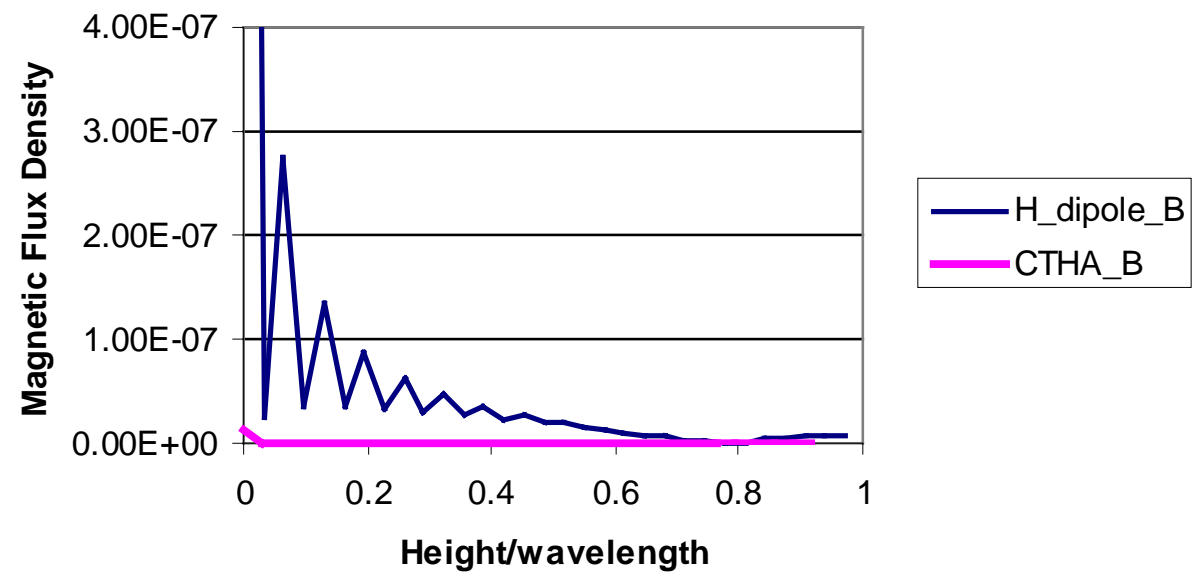

Figure 33 Comparison of Magnetic Flux Density

From the view of the near field energy distribution of the antennas, it can be concluded that the CTHA will have smaller interaction with the metallic ground plane underneath. Consequently, the input impedance of the CTHA will not be affected as much as that of the horizontal dipole by the metallic ground plane in the vicinity.

\section{Summary}

From the above comparisons, it is clear that the CTHA has the advantage of being relatively impedance invariant to adjacent metal plate as compared to the other antennas. This is important because it suggests a smaller increase of SWR when foreign objects are in the vicinity of the CTHA.

The comparison of the impedance change of the CTHA with the single helical toroid antenna indicates that the coupling of the contrawound helices of the CTHA helps to explain the relative invariance of CTHA's impedance to the adjacent object. The comparison of the near field energy distribution under the horizontal dipole and the CTHA indicates that the CTHA has its near field energy more concentrated around the wires, so that the interaction between the CTHA and the 
adjacent object is expected to be relatively weaker. This provides another reason to explain the relative impedance invariance of the CTHA. 


\section{Chapter 7 Conclusions and Recommendations}

\section{Conclusions}

A study of the impedance effects of a metallic ground plane on the CTHA has been conducted numerically and experimentally. Both numerical and experimental results indicate that the CTHA, similar to the other antennas such as the vertical dipole, the horizontal dipole, and the horizontal loop, has its resistance and reactance vary as a damped sinusoid function of the distance between the CTHA and the metallic ground plane reference to the free space wavelength. The relative impedance change of the CTHA decreases along a spiral curve as the distance between the CTHA and the metallic ground plane increases.

The importance of this study is that it provides guidance in understanding how a CTHA's impedance changes as a function of the distance between the antenna and an adjacent object. By accurately knowing this function, a desired height can be found at which the CTHA can maintain good matching even if there were an object in the vicinity.

As the experimental results indicate, the CTHA has the advantage of being relatively impedance invariant in comparison with the other antennas when there is an object in the vicinity. This property indicates a smaller increase of the SWR.

Some study has been made to explore the reasons for CTHA's relative impedance invariance. The coupling of the two contrawound helices of the CTHA could be one of the reasons, and that the near field energy being more concentrated around the wires of the CTHA could be another.

The agreement between the NEC results and the experimental results for the vertical dipole, the horizontal dipole and the horizontal loop indicate that NEC is an effective numerical tool for 
studying the antennas' impedance and modeling metallic surfaces. But being unable to model the core materials with dielectric constant other than one, NEC can not calculate the CTHA's impedance accurately.

XFDTD is a better numerical tool for modeling the CTHA comparing with NEC. But as a compromise to the accuracy of calculations, the calculating time and required memory size of XFDTD are much greater than those of NEC.

\section{Recommendations}

Further study, especially some theoretical analysis on the mechanism of coupling between the antenna and the adjacent objects, should be made so that a better understanding of the antenna's impedance change can be obtained. This may provide a better solution for the mismatching problem of antennas due to the objects in the vicinity.

This study has disclosed the relation between the magnitude of the relative impedance change and the SWR of the antennas. But the direct relation between the magnitude of the relative impedance change of the CTHA and the matching network has not been studied. It is believed that further study of this relationship may be beneficial to solving the mismatching problems of the CTHA. This mismatching is conjectured to be related to an interaction of the fields of the matching network and the CTHA. It is suggested that this needs further study. 


\section{References}

1. "The IEEE Standard Definitions of Terms for Antennas," IEEE Transactions on Antennas and Propagation, Vol. AP-31, No.6, Part II, November, 1983.

2. Rudge, A. W., Milne, K., Olver, A. D., and Knight, P., The Handbook of Antenna Design, Peter Peregrinus Ltd., Vol. 1, 1982.

3. Macnamara, T., Handbook of Antennas for EMC, Artech House, Inc., 1995.

4. Van Voorhis K. L. and Smith, J. E., “Toroidal Antenna," United States Patent Number 5,442,369, August 15, 1995.

5. Smith, J. E., “Contrawound Antenna," United States Patent Number 5,654,723, August 5, 1997.

6. Parsons, J., "Toroidal Helical Antenna (THA), Development Project," Technical Report, Wintec, Inc., June 1994.

7. Ellithy, W. S., "The Toroidal Helical Antenna Analysis and Performance Evaluation," Ph.D Dissertation, WVU, Nov. 1996.

8. Balanis, C.A., Antenna Theory - Analysis and Design, Second Edition, John Wiley \& Sons, Inc., 1997.

9. Babu, R. C., "Impedance Calculations for Horizontal Wire Antennas above a Loosy Earth," Journal of Electromagnetic Waves and Applications, Vol. 11, P1567-1591, 1997.

10. Collin, R. E. and Bucker, F. J., Antenna Theory, Part II, McGraw-Hill, 1969.

11. Burke, G. J., "Numerical Electromagnetics Code - Method of Moments," Lawrence Livermore National Laboratory, 1992.

12. Vogler, L. E. and Noble, J. L., "Curves of Input Impedance Change due to Ground for Dipole Antennas," U. S. National Bureau of Standards, Monograph 72, 1964.

13. Craven, R. P. M., "CTHA Feed Study 1," CTHA Internal Report Series 980325, Center for Industrial Research Application, West Virginia University, Aug. 1998.

14. Moore, J. and Pizer, R., Moment Methods in Electromagnetics, Techniques and Applications, Research Studies Press LTD., John Wiley \& Sons Inc., 1984.

15. Kraus, J. D., Antennas, Second Edition, McGraw-Hill Inc., 1988.

16. Pertl, F. A., "Expected Anechoic chamber Properties", CTHA Internal Report Series 980622, Center for Industrial Research Applications, West Virginia University, June, 1998. 
17. "User's Guide-HP 8753D Option 011 Network Analyzer," Hewlett-Packard Company, 1994.

18. Paul, C. R. and Nasar, S. A., Introduction to Electromagnetic Fields, McGraw-Hill Book Company, 1987.

19. Ostle, B. and Mensing, R. W., Statistics in Research, Iowa State University Press, 1975.

20. "User's Manual for XFDTD, the X-Window Finite Difference Time Domain Graphical User Interface for Electromagnetic Calculations," Version 4.0, Remcom, Inc, March 1997.

21. Straw, R. D., Hall, G. L., and Beezley, B., The ARRL Antenna Book, The American Radio Relay League, 1994.

22. Cheng, D. K., Field and Wave Electromagnetics, Second Edition, Addison-Wesley Publishing Company, 1992. 


\section{Appendix A Numerical Model of Antennas}

1. The NEC Cards for Modeling the Horizontal CTHA at $1 \mathrm{~mm}$ above the 3ft $x$ 3ft Metal Plate

GW 13 2.587E-2 0.000E+0 5.091E-3 2.747E-2 0.000E+0 5.091E-3 3.62E-4 GW 21 2.587E-2 0.000E+0 5.091E-3 2.508E-2 7.883E-4 4.842E-3 3.62E-4 GW 31 2.508E-2 7.883E-4 4.842E-3 2.363E-2 1.487E-3 4.119E-3 3.62E-4 GW 41 2.363E-2 1.487E-3 4.119E-3 2.245E-2 2.122E-3 2.993E-3 3.62E-4 GW 51 2.245E-2 2.122E-3 2.993E-3 2.166E-2 2.736E-3 1.573E-3 3.62E-4 GW 61 2.166E-2 2.736E-3 1.573E-3 2.131E-2 3.376E-3 6.235E-19 3.62E-4 GW 71 2.131E-2 3.376E-3 6.235E-19 2.144E-2 4.090E-3 -1.573E-3 3.62E-4 GW 81 2.144E-2 4.090E-3 -1.573E-3 2.201E-2 4.919E-3 -2.993E-3 3.62E-4 GW 91 2.201E-2 4.919E-3 -2.993E-3 2.293E-2 5.888E-3 -4.119E-3 3.62E-4 GW 101 2.293E-2 5.888E-3 -4.119E-3 2.410E-2 7.002E-3 -4.842E-3 3.62E-4 GW $1112.410 \mathrm{E}-2$ 7.002E-3 -4.842E-3 2.536E-2 8.241E-3 -5.091E-3 3.62E-4 GW 121 2.536E-2 8.241E-3 -5.091E-3 2.657E-2 9.567E-3 -4.842E-3 3.62E-4 GW 131 2.657E-2 9.567E-3 -4.842E-3 2.758E-2 1.092E-2 -4.119E-3 3.62E-4 GW 141 2.758E-2 1.092E-2 -4.119E-3 2.826E-2 1.223E-2 -2.993E-3 3.62E-4 GW 15 12.826E-2 1.223E-2 -2.993E-3 2.851E-2 1.342E-2 -1.573E-3 3.62E-4 GW $1612.851 \mathrm{E}-2$ 1.342E-2 -1.573E-3 2.830E-2 1.442E-2 -1.247E-18 3.62E-4 GW 171 2.830E-2 1.442E-2 -1.247E-18 2.761E-2 1.518E-2 1.973E-3 3.62E-4 GW 181 2.761E-2 1.518E-2 1.973E-3 2.650E-2 1.567E-2 3.753E-3 3.62E-4 GW 191 2.650E-2 1.567E-2 3.753E-3 2.505E-2 1.589E-2 5.165E-3 3.62E-4 GW 201 2.505E-2 1.589E-2 5.165E-3 2.336E-2 1.588E-2 6.072E-3 3.62E-4 GW 21 1 2.336E-2 1.588E-2 6.072E-3 2.158E-2 1.568E-2 6.384E-3 3.62E-4 GW 221 2.158E-2 1.568E-2 6.384E-3 1.983E-2 1.538E-2 6.072E-3 3.62E-4 GW 231 1.983E-2 1.538E-2 6.072E-3 1.824E-2 1.509E-2 5.165E-3 3.62E-4 GW 241 1.824E-2 1.509E-2 5.165E-3 1.692E-2 1.491E-2 3.753E-3 3.62E-4 GW 251 1.692E-2 1.491E-2 3.753E-3 1.591E-2 1.494E-2 1.973E-3 3.62E-4 GW 261 1.591E-2 1.494E-2 1.973E-3 1.526E-2 1.526E-2 1.870E-18 3.62E-4 
GW 271 1.526E-2 1.526E-2 1.870E-18 1.494E-2 1.591E-2 -1.573E-3 3.62E-4 GW 281 1.494E-2 1.591E-2 -1.573E-3 1.491E-2 1.692E-2 -2.993E-3 3.62E-4 GW 291 1.491E-2 1.692E-2 -2.993E-3 1.509E-2 1.824E-2 -4.119E-3 3.62E-4 GW 301 1.509E-2 1.824E-2 -4.119E-3 1.538E-2 1.983E-2 -4.842E-3 3.62E-4 GW 311 1.538E-2 1.983E-2 -4.842E-3 1.568E-2 2.158E-2 -5.091E-3 3.62E-4 GW 321 1.568E-2 2.158E-2 -5.091E-3 1.588E-2 2.336E-2 -4.842E-3 3.62E-4 GW 331 1.588E-2 2.336E-2 -4.842E-3 1.589E-2 2.505E-2 -4.119E-3 3.62E-4 GW 341 1.589E-2 2.505E-2 -4.119E-3 1.567E-2 2.650E-2 -2.993E-3 3.62E-4 GW 351 1.567E-2 2.650E-2 -2.993E-3 1.518E-2 2.761E-2 -1.573E-3 3.62E-4 GW 361 1.518E-2 2.761E-2 -1.573E-3 1.442E-2 2.830E-2 -2.494E-18 3.62E-4 GW 371 1.442E-2 2.830E-2 -2.494E-18 1.342E-2 2.851E-2 1.973E-3 3.62E-4 GW 381 1.342E-2 2.851E-2 1.973E-3 1.223E-2 2.826E-2 3.753E-3 3.62E-4 GW 391 1.223E-2 2.826E-2 3.753E-3 1.092E-2 2.758E-2 5.165E-3 3.62E-4 GW 401 1.092E-2 2.758E-2 5.165E-3 9.567E-3 2.657E-2 6.072E-3 3.62E-4 GW 41 1 9.567E-3 2.657E-2 6.072E-3 8.241E-3 2.536E-2 6.384E-3 3.62E-4 GW 421 8.241E-3 2.536E-2 6.384E-3 7.002E-3 2.410E-2 6.072E-3 3.62E-4 GW 43 1 7.002E-3 2.410E-2 6.072E-3 5.888E-3 2.293E-2 5.165E-3 3.62E-4 GW 441 5.888E-3 2.293E-2 5.165E-3 4.919E-3 2.201E-2 3.753E-3 3.62E-4 GW 45 14.919E-3 2.201E-2 3.753E-3 4.090E-3 2.144E-2 1.973E-3 3.62E-4 GW 461 4.090E-3 2.144E-2 1.973E-3 3.376E-3 2.131E-2 3.117E-18 3.62E-4 GW 471 3.376E-3 2.131E-2 3.117E-18 2.736E-3 2.166E-2 -1.573E-3 3.62E-4 GW 481 2.736E-3 2.166E-2 -1.573E-3 2.122E-3 2.245E-2 -2.993E-3 3.62E-4 GW 491 2.122E-3 2.245E-2 -2.993E-3 1.487E-3 2.363E-2 -4.119E-3 3.62E-4 GW 501 1.487E-3 2.363E-2 -4.119E-3 7.883E-4 2.508E-2 -4.842E-3 3.62E-4 GW 51 1 7.883E-4 2.508E-2 -4.842E-3 1.633E-18 2.667E-2 -5.091E-3 3.62E-4 GW 521 1.633E-18 2.667E-2 -5.091E-3 -8.871E-4 2.823E-2 -4.842E-3 3.62E-4 GW 53 1 -8.871E-4 2.823E-2 -4.842E-3 -1.863E-3 2.960E-2 -4.119E-3 3.62E-4 GW 54 -1.863E-3 2.960E-2 -4.119E-3 -2.898E-3 3.065E-2 -2.993E-3 3.62E-4 GW 551 -2.898E-3 3.065E-2 -2.993E-3 -3.950E-3 3.126E-2 -1.573E-3 3.62E-4 GW 56 1 -3.950E-3 3.126E-2 -1.573E-3 -4.969E-3 3.137E-2 -1.279E-17 3.62E-4 GW 57 -4.969E-3 3.137E-2 -1.279E-17 -5.905E-3 3.095E-2 1.973E-3 3.62E-4 GW 581 -5.905E-3 3.095E-2 1.973E-3 -6.716E-3 3.005E-2 3.753E-3 3.62E-4 
GW 591 -6.716E-3 3.005E-2 3.753E-3 -7.377E-3 2.873E-2 5.165E-3 3.62E-4 GW 601 -7.377E-3 2.873E-2 5.165E-3 -7.880E-3 2.712E-2 6.072E-3 3.62E-4 GW 611 -7.880E-3 2.712E-2 6.072E-3 -8.241E-3 2.536E-2 6.384E-3 3.62E-4 GW 621 -8.241E-3 2.536E-2 6.384E-3 -8.501E-3 2.361E-2 6.072E-3 3.62E-4 GW 631 -8.501E-3 2.361E-2 6.072E-3 -8.716E-3 2.201E-2 5.165E-3 3.62E-4 GW 641 -8.716E-3 2.201E-2 5.165E-3 -8.956E-3 2.070E-2 3.753E-3 3.62E-4 GW 651 -8.956E-3 2.070E-2 3.753E-3 -9.294E-3 1.975E-2 1.973E-3 3.62E-4 GW 661 -9.294E-3 1.975E-2 1.973E-3 -9.796E-3 1.923E-2 -4.680E-18 3.62E-4 GW 67 - $-9.796 \mathrm{E}-3$ 1.923E-2 -4.680E-18 -1.052E-2 1.913E-2 -1.573E-3 3.62E-4 GW 68 -1.052E-2 1.913E-2 -1.573E-3 -1.148E-2 1.941E-2 -2.993E-3 3.62E-4 GW 69 1 -1.148E-2 1.941E-2 -2.993E-3 -1.269E-2 1.999E-2 -4.119E-3 3.62E-4 GW 701 -1.269E-2 1.999E-2 -4.119E-3 -1.411E-2 2.076E-2 -4.842E-3 3.62E-4 GW 71 -1.411E-2 2.076E-2 -4.842E-3 -1.568E-2 2.158E-2 -5.091E-3 3.62E-4 GW 721 -1.568E-2 2.158E-2 -5.091E-3 -1.731E-2 2.232E-2 -4.842E-3 3.62E-4 GW 73 1 -1.731E-2 2.232E-2 -4.842E-3 -1.891E-2 2.286E-2 -4.119E-3 3.62E-4 GW 74 1 -1.891E-2 2.286E-2 -4.119E-3 -2.036E-2 2.310E-2 -2.993E-3 3.62E-4 GW 751 -2.036E-2 2.310E-2 -2.993E-3 -2.157E-2 2.297E-2 -1.573E-3 3.62E-4 GW 761 -2.157E-2 2.297E-2 -1.573E-3 -2.246E-2 2.246E-2 -1.403E-17 3.62E-4 GW 77 1 -2.246E-2 2.246E-2 -1.403E-17 -2.297E-2 2.157E-2 1.973E-3 3.62E-4 GW 78 -2.297E-2 2.157E-2 1.973E-3 -2.310E-2 2.036E-2 3.753E-3 3.62E-4 GW 791 -2.310E-2 2.036E-2 3.753E-3 -2.286E-2 1.891E-2 5.165E-3 3.62E-4 GW 801 -2.286E-2 1.891E-2 5.165E-3 -2.232E-2 1.731E-2 6.072E-3 3.62E-4 GW 811 -2.232E-2 1.731E-2 6.072E-3 -2.158E-2 1.568E-2 6.384E-3 3.62E-4 GW 821 -2.158E-2 1.568E-2 6.384E-3 -2.076E-2 1.411E-2 6.072E-3 3.62E-4 GW 831 -2.076E-2 1.411E-2 6.072E-3 -1.999E-2 1.269E-2 5.165E-3 3.62E-4 GW 841 -1.999E-2 1.269E-2 5.165E-3 -1.941E-2 1.148E-2 3.753E-3 3.62E-4 GW 851 -1.941E-2 1.148E-2 3.753E-3 -1.913E-2 1.052E-2 1.973E-3 3.62E-4 GW 861 -1.913E-2 1.052E-2 1.973E-3 -1.923E-2 9.796E-3 -3.433E-18 3.62E-4 GW 87 -1.923E-2 9.796E-3 -3.433E-18 -1.975E-2 9.294E-3 -1.573E-3 3.62E-4 GW 88 1 -1.975E-2 9.294E-3 -1.573E-3 -2.070E-2 8.956E-3 -2.993E-3 3.62E-4 GW 89 1 -2.070E-2 8.956E-3 -2.993E-3 -2.201E-2 8.716E-3 -4.119E-3 3.62E-4 GW 901 -2.201E-2 8.716E-3 -4.119E-3 -2.361E-2 8.501E-3 -4.842E-3 3.62E-4 
GW 91 -2.361E-2 8.501E-3 -4.842E-3 -2.536E-2 8.241E-3 -5.091E-3 3.62E-4 GW 921 -2.536E-2 8.241E-3 -5.091E-3 -2.712E-2 7.880E-3 -4.842E-3 3.62E-4 GW 93 1 -2.712E-2 7.880E-3 -4.842E-3 -2.873E-2 7.377E-3 -4.119E-3 3.62E-4 GW 94 1 -2.873E-2 7.377E-3 -4.119E-3 -3.005E-2 6.716E-3 -2.993E-3 3.62E-4 GW 951 -3.005E-2 6.716E-3 -2.993E-3 -3.095E-2 5.905E-3 -1.573E-3 3.62E-4 GW 961 -3.095E-2 5.905E-3 -1.573E-3 -3.137E-2 4.969E-3 -1.528E-17 3.62E-4 GW 971 -3.137E-2 4.969E-3 -1.528E-17 -3.126E-2 3.950E-3 1.973E-3 3.62E-4 GW 981 -3.126E-2 3.950E-3 1.973E-3 -3.065E-2 2.898E-3 3.753E-3 3.62E-4 GW 991 -3.065E-2 2.898E-3 3.753E-3 -2.960E-2 1.863E-3 5.165E-3 3.62E-4 GW 1001 -2.960E-2 1.863E-3 5.165E-3 -2.823E-2 8.871E-4 6.072E-3 3.62E-4 GW 1011 -2.823E-2 8.871E-4 6.072E-3 -2.667E-2 3.266E-18 6.384E-3 3.62E-4 GW 1021 -2.667E-2 3.266E-18 6.384E-3 -2.508E-2 -7.883E-4 6.072E-3 3.62E-4 GW 1031 -2.508E-2 -7.883E-4 6.072E-3 -2.363E-2 -1.487E-3 5.165E-3 3.62E-4 GW 104 - 1 -2.363E-2 -1.487E-3 5.165E-3 -2.245E-2 -2.122E-3 3.753E-3 3.62E-4 GW 1051 -2.245E-2 -2.122E-3 3.753E-3 -2.166E-2 -2.736E-3 1.973E-3 3.62E-4 GW 106 1 -2.166E-2 -2.736E-3 1.973E-3 -2.131E-2 -3.376E-3 -2.186E-18 3.62E-4 GW 107 - $2.131 \mathrm{E}-2$-3.376E-3 -2.186E-18 -2.144E-2 -4.090E-3 -1.573E-3 3.62E-4 GW 108 1 -2.144E-2 -4.090E-3 -1.573E-3 -2.201E-2 -4.919E-3 -2.993E-3 3.62E-4 GW 109 1 -2.201E-2 -4.919E-3 -2.993E-3 -2.293E-2 -5.888E-3 -4.119E-3 3.62E-4 GW 1101 -2.293E-2 -5.888E-3 -4.119E-3 -2.410E-2 -7.002E-3 -4.842E-3 3.62E-4 GW 1111 -2.410E-2 -7.002E-3 -4.842E-3 -2.536E-2 -8.241E-3 -5.091E-3 3.62E-4 GW 1121 -2.536E-2 -8.241E-3 -5.091E-3 -2.657E-2 -9.567E-3 -4.842E-3 3.62E-4 GW 1131 -2.657E-2 -9.567E-3 -4.842E-3 -2.758E-2 -1.092E-2 -4.119E-3 3.62E-4 GW 1141 -2.758E-2 -1.092E-2 -4.119E-3 -2.826E-2 -1.223E-2 -2.993E-3 3.62E-4 GW 1151 -2.826E-2 -1.223E-2 -2.993E-3 -2.851E-2 -1.342E-2 -1.573E-3 3.62E-4 GW 1161 -2.851E-2 -1.342E-2 -1.573E-3 -2.830E-2 -1.442E-2 -1.653E-17 3.62E-4 GW 1171 -2.830E-2 -1.442E-2 -1.653E-17 -2.761E-2 -1.518E-2 1.973E-3 3.62E-4 GW 1181 -2.761E-2 -1.518E-2 1.973E-3 -2.650E-2 -1.567E-2 3.753E-3 3.62E-4 GW 1191 -2.650E-2 -1.567E-2 3.753E-3 -2.505E-2 -1.589E-2 5.165E-3 3.62E-4 GW 1201 -2.505E-2 -1.589E-2 5.165E-3 -2.336E-2 -1.588E-2 6.072E-3 3.62E-4 GW 1211 -2.336E-2 -1.588E-2 6.072E-3 -2.158E-2 -1.568E-2 6.384E-3 3.62E-4 GW 1221 -2.158E-2 -1.568E-2 6.384E-3 -1.983E-2 -1.538E-2 6.072E-3 3.62E-4 
GW 1231 -1.983E-2 -1.538E-2 6.072E-3 -1.824E-2 -1.509E-2 5.165E-3 3.62E-4 GW 1241 -1.824E-2 -1.509E-2 5.165E-3 -1.692E-2 -1.491E-2 3.753E-3 3.62E-4 GW 1251 -1.692E-2 -1.491E-2 3.753E-3 -1.591E-2 -1.494E-2 1.973E-3 3.62E-4 GW 1261 -1.591E-2 -1.494E-2 1.973E-3 -1.526E-2 -1.526E-2 -9.387E-19 3.62E-4 GW 1271 -1.526E-2 -1.526E-2 -9.387E-19 -1.494E-2 -1.591E-2 -1.573E-3 3.62E-4 GW 1281 -1.494E-2 -1.591E-2 -1.573E-3 -1.491E-2 -1.692E-2 -2.993E-3 3.62E-4 GW 129 1 -1.491E-2 -1.692E-2 -2.993E-3 -1.509E-2 -1.824E-2 -4.119E-3 3.62E-4 GW 1301 -1.509E-2 -1.824E-2 -4.119E-3 -1.538E-2 -1.983E-2 -4.842E-3 3.62E-4 GW 1311 -1.538E-2 -1.983E-2 -4.842E-3 -1.568E-2 -2.158E-2 -5.091E-3 3.62E-4 GW 1321 -1.568E-2 -2.158E-2 -5.091E-3 -1.588E-2 -2.336E-2 -4.842E-3 3.62E-4 GW 1331 -1.588E-2 -2.336E-2 -4.842E-3 -1.589E-2 -2.505E-2 -4.119E-3 3.62E-4 GW 1341 -1.589E-2 -2.505E-2 -4.119E-3 -1.567E-2 -2.650E-2 -2.993E-3 3.62E-4 GW 1351 -1.567E-2 -2.650E-2 -2.993E-3 -1.518E-2 -2.761E-2 -1.573E-3 3.62E-4 GW 1361 -1.518E-2 -2.761E-2 -1.573E-3 -1.442E-2 -2.830E-2 -1.777E-17 3.62E-4 GW 137 -1.442E-2 -2.830E-2 -1.777E-17 -1.342E-2 -2.851E-2 1.973E-3 3.62E-4 GW 1381 -1.342E-2 -2.851E-2 1.973E-3 -1.223E-2 -2.826E-2 3.753E-3 3.62E-4 GW 1391 -1.223E-2 -2.826E-2 3.753E-3 -1.092E-2 -2.758E-2 5.165E-3 3.62E-4 GW 1401 -1.092E-2 -2.758E-2 5.165E-3 -9.567E-3 -2.657E-2 6.072E-3 3.62E-4 GW 1411 -9.567E-3 -2.657E-2 6.072E-3 -8.241E-3 -2.536E-2 6.384E-3 3.62E-4 GW 1421 -8.241E-3 -2.536E-2 6.384E-3 -7.002E-3 -2.410E-2 6.072E-3 3.62E-4 GW 143 1 -7.002E-3 -2.410E-2 6.072E-3 -5.888E-3 -2.293E-2 5.165E-3 3.62E-4 GW 1441 -5.888E-3 -2.293E-2 5.165E-3 -4.919E-3 -2.201E-2 3.753E-3 3.62E-4 GW 1451 -4.919E-3 -2.201E-2 3.753E-3 -4.090E-3 -2.144E-2 1.973E-3 3.62E-4 GW 1461 -4.090E-3 -2.144E-2 1.973E-3 -3.376E-3 -2.131E-2 3.083E-19 3.62E-4 GW 147 1 -3.376E-3 -2.131E-2 3.083E-19 -2.736E-3 -2.166E-2 -1.573E-3 3.62E-4 GW 148 1 -2.736E-3 -2.166E-2 -1.573E-3 -2.122E-3 -2.245E-2 -2.993E-3 3.62E-4 GW 149 1 -2.122E-3 -2.245E-2 -2.993E-3 -1.487E-3 -2.363E-2 -4.119E-3 3.62E-4 GW 1501 -1.487E-3 -2.363E-2 -4.119E-3 -7.883E-4 -2.508E-2 -4.842E-3 3.62E-4 GW 151 1 -7.883E-4 -2.508E-2 -4.842E-3 -4.899E-18 -2.667E-2 -5.091E-3 3.62E-4 GW 1521 -4.899E-18 -2.667E-2 -5.091E-3 8.871E-4 -2.823E-2 -4.842E-3 3.62E-4 GW 1531 8.871E-4 -2.823E-2 -4.842E-3 1.863E-3 -2.960E-2 -4.119E-3 3.62E-4 GW 1541 1.863E-3 -2.960E-2 -4.119E-3 2.898E-3 -3.065E-2 -2.993E-3 3.62E-4 
GW 1551 2.898E-3 -3.065E-2 -2.993E-3 3.950E-3 -3.126E-2 -1.573E-3 3.62E-4 GW 1561 3.950E-3 -3.126E-2 -1.573E-3 4.969E-3 -3.137E-2 -1.902E-17 3.62E-4 GW 157 14.969E-3 -3.137E-2 -1.902E-17 5.905E-3 -3.095E-2 1.973E-3 3.62E-4 GW 1581 5.905E-3 -3.095E-2 1.973E-3 6.716E-3 -3.005E-2 3.753E-3 3.62E-4 GW 1591 6.716E-3 -3.005E-2 3.753E-3 7.377E-3 -2.873E-2 5.165E-3 3.62E-4 GW 1601 7.377E-3 -2.873E-2 5.165E-3 7.880E-3 -2.712E-2 6.072E-3 3.62E-4 GW 1611 7.880E-3 -2.712E-2 6.072E-3 8.241E-3 -2.536E-2 6.384E-3 3.62E-4 GW 1621 8.241E-3 -2.536E-2 6.384E-3 8.501E-3 -2.361E-2 6.072E-3 3.62E-4 GW 1631 8.501E-3 -2.361E-2 6.072E-3 8.716E-3 -2.201E-2 5.165E-3 3.62E-4 GW 1641 8.716E-3 -2.201E-2 5.165E-3 8.956E-3 -2.070E-2 3.753E-3 3.62E-4 GW 1651 8.956E-3 -2.070E-2 3.753E-3 9.294E-3 -1.975E-2 1.973E-3 3.62E-4 GW 1661 9.294E-3 -1.975E-2 1.973E-3 9.796E-3 -1.923E-2 1.555E-18 3.62E-4 GW 167 19.796E-3 -1.923E-2 1.555E-18 1.052E-2 -1.913E-2 -1.573E-3 3.62E-4 GW 1681 1.052E-2 -1.913E-2 -1.573E-3 1.148E-2 -1.941E-2 -2.993E-3 3.62E-4 GW 1691 1.148E-2 -1.941E-2 -2.993E-3 1.269E-2 -1.999E-2 -4.119E-3 3.62E-4 GW 1701 1.269E-2 -1.999E-2 -4.119E-3 1.411E-2 -2.076E-2 -4.842E-3 3.62E-4 GW 171 1.411E-2 -2.076E-2 -4.842E-3 1.568E-2 -2.158E-2 -5.091E-3 3.62E-4 GW 1721 1.568E-2 -2.158E-2 -5.091E-3 1.731E-2 -2.232E-2 -4.842E-3 3.62E-4 GW 1731 1.731E-2 -2.232E-2 -4.842E-3 1.891E-2 -2.286E-2 -4.119E-3 3.62E-4 GW 1741 1.891E-2 -2.286E-2 -4.119E-3 2.036E-2 -2.310E-2 -2.993E-3 3.62E-4 GW $17512.036 \mathrm{E}-2$-2.310E-2 -2.993E-3 2.157E-2 -2.297E-2 -1.573E-3 3.62E-4 GW 1761 2.157E-2 -2.297E-2 -1.573E-3 2.246E-2 -2.246E-2 -2.027E-17 3.62E-4 GW 1771 2.246E-2 -2.246E-2 -2.027E-17 2.297E-2 -2.157E-2 1.973E-3 3.62E-4 GW 1781 2.297E-2 -2.157E-2 1.973E-3 2.310E-2 -2.036E-2 3.753E-3 3.62E-4 GW 1791 2.310E-2 -2.036E-2 3.753E-3 2.286E-2 -1.891E-2 5.165E-3 3.62E-4 GW 1801 2.286E-2 -1.891E-2 5.165E-3 2.232E-2 -1.731E-2 6.072E-3 3.62E-4 GW 1811 2.232E-2 -1.731E-2 6.072E-3 2.158E-2 -1.568E-2 6.384E-3 3.62E-4 GW 1821 2.158E-2 -1.568E-2 6.384E-3 2.076E-2 -1.411E-2 6.072E-3 3.62E-4 GW $18312.076 \mathrm{E}-2$-1.411E-2 6.072E-3 1.999E-2 -1.269E-2 5.165E-3 3.62E-4 GW 1841 1.999E-2 -1.269E-2 5.165E-3 1.941E-2 -1.148E-2 3.753E-3 3.62E-4 GW 1851 1.941E-2 -1.148E-2 3.753E-3 1.913E-2 -1.052E-2 1.973E-3 3.62E-4 GW 1861 1.913E-2 -1.052E-2 1.973E-3 1.923E-2 -9.796E-3 2.802E-18 3.62E-4 
GW 187 1 1.923E-2 -9.796E-3 2.802E-18 1.975E-2 -9.294E-3 -1.573E-3 3.62E-4 GW 1881 1.975E-2 -9.294E-3 -1.573E-3 2.070E-2 -8.956E-3 -2.993E-3 3.62E-4 GW 1891 2.070E-2 -8.956E-3 -2.993E-3 2.201E-2 -8.716E-3 -4.119E-3 3.62E-4 GW 1901 2.201E-2 -8.716E-3 -4.119E-3 2.361E-2 -8.501E-3 -4.842E-3 3.62E-4 GW $19112.361 \mathrm{E}-2$-8.501E-3 -4.842E-3 2.536E-2 -8.241E-3 -5.091E-3 3.62E-4 GW 1921 2.536E-2 -8.241E-3 -5.091E-3 2.712E-2 -7.880E-3 -4.842E-3 3.62E-4 GW 1931 2.712E-2 -7.880E-3 -4.842E-3 2.873E-2 -7.377E-3 -4.119E-3 3.62E-4 GW 1941 2.873E-2 -7.377E-3 -4.119E-3 3.005E-2 -6.716E-3 -2.993E-3 3.62E-4 GW 1951 3.005E-2 -6.716E-3 -2.993E-3 3.095E-2 -5.905E-3 -1.573E-3 3.62E-4 GW 1961 3.095E-2 -5.905E-3 -1.573E-3 3.137E-2 -4.969E-3 -2.151E-17 3.62E-4 GW 197 1 3.137E-2 -4.969E-3 -2.151E-17 3.126E-2 -3.950E-3 1.573E-3 3.62E-4 GW 1981 3.126E-2 -3.950E-3 1.573E-3 3.065E-2 -2.898E-3 2.993E-3 3.62E-4 GW 1991 3.065E-2 -2.898E-3 2.993E-3 2.960E-2 -1.863E-3 4.119E-3 3.62E-4 GW 2001 2.960E-2 -1.863E-3 4.119E-3 2.823E-2 -8.871E-4 4.842E-3 3.62E-4 GW 2011 2.823E-2 -8.871E-4 4.842E-3 2.747E-2 0.000E+0 5.091E-3 3.62E-4 GW 2021 2.747E-2 0.000E+0 5.091E-3 2.823E-2 8.871E-4 4.842E-3 3.62E-4 GW 2031 2.823E-2 8.871E-4 4.842E-3 2.960E-2 1.863E-3 4.119E-3 3.62E-4 GW 2041 2.960E-2 1.863E-3 4.119E-3 3.065E-2 2.898E-3 2.993E-3 3.62E-4 GW 2051 3.065E-2 2.898E-3 2.993E-3 3.126E-2 3.950E-3 1.573E-3 3.62E-4 GW 2061 3.126E-2 3.950E-3 1.573E-3 3.137E-2 4.969E-3 -5.266E-36 3.62E-4 GW 2071 3.137E-2 4.969E-3 -5.266E-36 3.095E-2 5.905E-3 -1.973E-3 3.62E-4 GW 2081 3.095E-2 5.905E-3 -1.973E-3 3.005E-2 6.716E-3 -3.753E-3 3.62E-4 GW 2091 3.005E-2 6.716E-3 -3.753E-3 2.873E-2 7.377E-3 -5.165E-3 3.62E-4 GW 2101 2.873E-2 7.377E-3 -5.165E-3 2.712E-2 7.880E-3 -6.072E-3 3.62E-4 GW 2111 2.712E-2 7.880E-3 -6.072E-3 2.536E-2 8.241E-3 -6.384E-3 3.62E-4 GW 2121 2.536E-2 8.241E-3 -6.384E-3 2.361E-2 8.501E-3 -6.072E-3 3.62E-4 GW 2131 2.361E-2 8.501E-3 -6.072E-3 2.201E-2 8.716E-3 -5.165E-3 3.62E-4 GW 2141 2.201E-2 8.716E-3 -5.165E-3 2.070E-2 8.956E-3 -3.753E-3 3.62E-4 GW 2151 2.070E-2 8.956E-3 -3.753E-3 1.975E-2 9.294E-3 -1.973E-3 3.62E-4 GW 2161 1.975E-2 9.294E-3 -1.973E-3 1.923E-2 9.796E-3 -6.235E-19 3.62E-4 GW 2171 1.923E-2 9.796E-3 -6.235E-19 1.913E-2 1.052E-2 1.573E-3 3.62E-4 GW 2181 1.913E-2 1.052E-2 1.573E-3 1.941E-2 1.148E-2 2.993E-3 3.62E-4 
GW 2191 1.941E-2 1.148E-2 2.993E-3 1.999E-2 1.269E-2 4.119E-3 3.62E-4 GW 2201 1.999E-2 1.269E-2 4.119E-3 2.076E-2 1.411E-2 4.842E-3 3.62E-4 GW 2211 2.076E-2 1.411E-2 4.842E-3 2.158E-2 1.568E-2 5.091E-3 3.62E-4 GW 2221 2.158E-2 1.568E-2 5.091E-3 2.232E-2 1.731E-2 4.842E-3 3.62E-4 GW 2231 2.232E-2 1.731E-2 4.842E-3 2.286E-2 1.891E-2 4.119E-3 3.62E-4 GW 2241 2.286E-2 1.891E-2 4.119E-3 2.310E-2 2.036E-2 2.993E-3 3.62E-4 GW 2251 2.310E-2 2.036E-2 2.993E-3 2.297E-2 2.157E-2 1.573E-3 3.62E-4 GW 2261 2.297E-2 2.157E-2 1.573E-3 2.246E-2 2.246E-2 1.247E-18 3.62E-4 GW 2271 2.246E-2 2.246E-2 1.247E-18 2.157E-2 2.297E-2 -1.973E-3 3.62E-4 GW 2281 2.157E-2 2.297E-2 -1.973E-3 2.036E-2 2.310E-2 -3.753E-3 3.62E-4 GW 2291 2.036E-2 2.310E-2 -3.753E-3 1.891E-2 2.286E-2 -5.165E-3 3.62E-4 GW 2301 1.891E-2 2.286E-2 -5.165E-3 1.731E-2 2.232E-2 -6.072E-3 3.62E-4 GW 2311 1.731E-2 2.232E-2 -6.072E-3 1.568E-2 2.158E-2 -6.384E-3 3.62E-4 GW 2321 1.568E-2 2.158E-2 -6.384E-3 1.411E-2 2.076E-2 -6.072E-3 3.62E-4 GW 2331 1.411E-2 2.076E-2 -6.072E-3 1.269E-2 1.999E-2 -5.165E-3 3.62E-4 GW 2341 1.269E-2 1.999E-2 -5.165E-3 1.148E-2 1.941E-2 -3.753E-3 3.62E-4 GW 2351 1.148E-2 1.941E-2 -3.753E-3 1.052E-2 1.913E-2 -1.973E-3 3.62E-4 GW 2361 1.052E-2 1.913E-2 -1.973E-3 9.796E-3 1.923E-2 -1.870E-18 3.62E-4 GW 2371 9.796E-3 1.923E-2 -1.870E-18 9.294E-3 1.975E-2 1.573E-3 3.62E-4 GW 2381 9.294E-3 1.975E-2 1.573E-3 8.956E-3 2.070E-2 2.993E-3 3.62E-4 GW 2391 8.956E-3 2.070E-2 2.993E-3 8.716E-3 2.201E-2 4.119E-3 3.62E-4 GW 2401 8.716E-3 2.201E-2 4.119E-3 8.501E-3 2.361E-2 4.842E-3 3.62E-4 GW 2411 8.501E-3 2.361E-2 4.842E-3 8.241E-3 2.536E-2 5.091E-3 3.62E-4 GW 2421 8.241E-3 2.536E-2 5.091E-3 7.880E-3 2.712E-2 4.842E-3 3.62E-4 GW 2431 7.880E-3 2.712E-2 4.842E-3 7.377E-3 2.873E-2 4.119E-3 3.62E-4 GW 2441 7.377E-3 2.873E-2 4.119E-3 6.716E-3 3.005E-2 2.993E-3 3.62E-4 GW 2451 6.716E-3 3.005E-2 2.993E-3 5.905E-3 3.095E-2 1.573E-3 3.62E-4 GW 2461 5.905E-3 3.095E-2 1.573E-3 4.969E-3 3.137E-2 2.494E-18 3.62E-4 GW 2471 4.969E-3 3.137E-2 2.494E-18 3.950E-3 3.126E-2 -1.973E-3 3.62E-4 GW 2481 3.950E-3 3.126E-2 -1.973E-3 2.898E-3 3.065E-2 -3.753E-3 3.62E-4 GW 2491 2.898E-3 3.065E-2 -3.753E-3 1.863E-3 2.960E-2 -5.165E-3 3.62E-4 GW 2501 1.863E-3 2.960E-2 -5.165E-3 8.871E-4 2.823E-2 -6.072E-3 3.62E-4 
GW 2511 8.871E-4 2.823E-2 -6.072E-3 1.633E-18 2.667E-2 -6.384E-3 3.62E-4 GW 2521 1.633E-18 2.667E-2 -6.384E-3 -7.883E-4 2.508E-2 -6.072E-3 3.62E-4 GW 2531 -7.883E-4 2.508E-2 -6.072E-3 -1.487E-3 2.363E-2 -5.165E-3 3.62E-4 GW 2541 -1.487E-3 2.363E-2 -5.165E-3 -2.122E-3 2.245E-2 -3.753E-3 3.62E-4 GW 2551 -2.122E-3 2.245E-2 -3.753E-3 -2.736E-3 2.166E-2 -1.973E-3 3.62E-4 GW 2561 -2.736E-3 2.166E-2 -1.973E-3 -3.376E-3 2.131E-2 -1.216E-17 3.62E-4 GW 2571 -3.376E-3 2.131E-2 -1.216E-17 -4.090E-3 2.144E-2 1.573E-3 3.62E-4 GW 2581 -4.090E-3 2.144E-2 1.573E-3 -4.919E-3 2.201E-2 2.993E-3 3.62E-4 GW 259 1 -4.919E-3 2.201E-2 2.993E-3 -5.888E-3 2.293E-2 4.119E-3 3.62E-4 GW 2601 -5.888E-3 2.293E-2 4.119E-3 -7.002E-3 2.410E-2 4.842E-3 3.62E-4 GW 2611 -7.002E-3 2.410E-2 4.842E-3 -8.241E-3 2.536E-2 5.091E-3 3.62E-4 GW 2621 -8.241E-3 2.536E-2 5.091E-3 -9.567E-3 2.657E-2 4.842E-3 3.62E-4 GW 2631 -9.567E-3 2.657E-2 4.842E-3 -1.092E-2 2.758E-2 4.119E-3 3.62E-4 GW 2641 -1.092E-2 2.758E-2 4.119E-3 -1.223E-2 2.826E-2 2.993E-3 3.62E-4 GW 2651 -1.223E-2 2.826E-2 2.993E-3 -1.342E-2 2.851E-2 1.573E-3 3.62E-4 GW 2661 -1.342E-2 2.851E-2 1.573E-3 -1.442E-2 2.830E-2 -5.303E-18 3.62E-4 GW 2671 -1.442E-2 2.830E-2 -5.303E-18 -1.518E-2 2.761E-2 -1.973E-3 3.62E-4 GW 2681 -1.518E-2 2.761E-2 -1.973E-3 -1.567E-2 2.650E-2 -3.753E-3 3.62E-4 GW 2691 -1.567E-2 2.650E-2 -3.753E-3 -1.589E-2 2.505E-2 -5.165E-3 3.62E-4 GW $2701-1.589 \mathrm{E}-2$ 2.505E-2 -5.165E-3 -1.588E-2 2.336E-2 -6.072E-3 3.62E-4 GW 2711 -1.588E-2 2.336E-2 -6.072E-3 -1.568E-2 2.158E-2 -6.384E-3 3.62E-4 GW $2721-1.568 \mathrm{E}-2$ 2.158E-2 -6.384E-3 -1.538E-2 1.983E-2 -6.072E-3 3.62E-4 GW 2731 -1.538E-2 1.983E-2 -6.072E-3 -1.509E-2 1.824E-2 -5.165E-3 3.62E-4 GW 2741 -1.509E-2 1.824E-2 -5.165E-3 -1.491E-2 1.692E-2 -3.753E-3 3.62E-4 GW 2751 -1.491E-2 1.692E-2 -3.753E-3 -1.494E-2 1.591E-2 -1.973E-3 3.62E-4 GW 2761 -1.494E-2 1.591E-2 -1.973E-3 -1.526E-2 1.526E-2 -1.341E-17 3.62E-4 GW 2771 -1.526E-2 1.526E-2 -1.341E-17 -1.591E-2 1.494E-2 1.573E-3 3.62E-4 GW 2781 -1.591E-2 1.494E-2 1.573E-3 -1.692E-2 1.491E-2 2.993E-3 3.62E-4 GW 2791 -1.692E-2 1.491E-2 2.993E-3 -1.824E-2 1.509E-2 4.119E-3 3.62E-4 GW 2801 -1.824E-2 1.509E-2 4.119E-3 -1.983E-2 1.538E-2 4.842E-3 3.62E-4 GW 2811 -1.983E-2 1.538E-2 4.842E-3 -2.158E-2 1.568E-2 5.091E-3 3.62E-4 GW 2821 -2.158E-2 1.568E-2 5.091E-3 -2.336E-2 1.588E-2 4.842E-3 3.62E-4 
GW 2831 -2.336E-2 1.588E-2 4.842E-3 -2.505E-2 1.589E-2 4.119E-3 3.62E-4 GW 2841 -2.505E-2 1.589E-2 4.119E-3 -2.650E-2 1.567E-2 2.993E-3 3.62E-4 GW 2851 -2.650E-2 1.567E-2 2.993E-3 -2.761E-2 1.518E-2 1.573E-3 3.62E-4 GW 2861 -2.761E-2 1.518E-2 1.573E-3 -2.830E-2 1.442E-2 -4.056E-18 3.62E-4 GW 287 1 -2.830E-2 1.442E-2 -4.056E-18 -2.851E-2 1.342E-2 -1.973E-3 3.62E-4 GW 2881 -2.851E-2 1.342E-2 -1.973E-3 -2.826E-2 1.223E-2 -3.753E-3 3.62E-4 GW $2891-2.826 \mathrm{E}-2$ 1.223E-2 -3.753E-3 -2.758E-2 1.092E-2 -5.165E-3 3.62E-4 GW 2901 -2.758E-2 1.092E-2 -5.165E-3 -2.657E-2 9.567E-3 -6.072E-3 3.62E-4 GW 2911 -2.657E-2 9.567E-3 -6.072E-3 -2.536E-2 8.241E-3 -6.384E-3 3.62E-4 GW 2921 -2.536E-2 8.241E-3 -6.384E-3 -2.410E-2 7.002E-3 -6.072E-3 3.62E-4 GW 293 1 -2.410E-2 7.002E-3 -6.072E-3 -2.293E-2 5.888E-3 -5.165E-3 3.62E-4 GW 2941 -2.293E-2 5.888E-3 -5.165E-3 -2.201E-2 4.919E-3 -3.753E-3 3.62E-4 GW 2951 -2.201E-2 4.919E-3 -3.753E-3 -2.144E-2 4.090E-3 -1.973E-3 3.62E-4 GW 2961 -2.144E-2 4.090E-3 -1.973E-3 -2.131E-2 3.376E-3 -1.466E-17 3.62E-4 GW 2971 -2.131E-2 3.376E-3 -1.466E-17 -2.166E-2 2.736E-3 1.573E-3 3.62E-4 GW 2981 -2.166E-2 2.736E-3 1.573E-3 -2.245E-2 2.122E-3 2.993E-3 3.62E-4 GW 299 1 -2.245E-2 2.122E-3 2.993E-3 -2.363E-2 1.487E-3 4.119E-3 3.62E-4 GW 3001 -2.363E-2 1.487E-3 4.119E-3 -2.508E-2 7.883E-4 4.842E-3 3.62E-4 GW 301 1 -2.508E-2 7.883E-4 4.842E-3 -2.667E-2 3.266E-18 5.091E-3 3.62E-4 GW 3021 -2.667E-2 3.266E-18 5.091E-3 -2.823E-2 -8.871E-4 4.842E-3 3.62E-4 GW 3031 -2.823E-2 -8.871E-4 4.842E-3 -2.960E-2 -1.863E-3 4.119E-3 3.62E-4 GW 3041 -2.960E-2 -1.863E-3 4.119E-3 -3.065E-2 -2.898E-3 2.993E-3 3.62E-4 GW 3051 -3.065E-2 -2.898E-3 2.993E-3 -3.126E-2 -3.950E-3 1.573E-3 3.62E-4 GW 306 1 -3.126E-2 -3.950E-3 1.573E-3 -3.137E-2 -4.969E-3 -2.809E-18 3.62E-4 GW 307 1 -3.137E-2 -4.969E-3 -2.809E-18 -3.095E-2 -5.905E-3 -1.973E-3 3.62E-4 GW 3081 -3.095E-2 -5.905E-3 -1.973E-3 -3.005E-2 -6.716E-3 -3.753E-3 3.62E-4 GW 3091 -3.005E-2 -6.716E-3 -3.753E-3 -2.873E-2 -7.377E-3 -5.165E-3 3.62E-4 GW 3101 -2.873E-2 -7.377E-3 -5.165E-3 -2.712E-2 -7.880E-3 -6.072E-3 3.62E-4 GW 311 1 -2.712E-2 -7.880E-3 -6.072E-3 -2.536E-2 -8.241E-3 -6.384E-3 3.62E-4 GW $3121-2.536 \mathrm{E}-2$-8.241E-3 -6.384E-3 -2.361E-2 -8.501E-3 -6.072E-3 3.62E-4 GW 3131 -2.361E-2 -8.501E-3 -6.072E-3 -2.201E-2 -8.716E-3 -5.165E-3 3.62E-4 GW 314 1 -2.201E-2 -8.716E-3 -5.165E-3 -2.070E-2 -8.956E-3 -3.753E-3 3.62E-4 
GW 3151 -2.070E-2 -8.956E-3 -3.753E-3 -1.975E-2 -9.294E-3 -1.973E-3 3.62E-4 GW 3161 -1.975E-2 -9.294E-3 -1.973E-3 -1.923E-2 -9.796E-3 -1.590E-17 3.62E-4 GW 317 -1.923E-2 -9.796E-3 -1.590E-17 -1.913E-2 -1.052E-2 1.573E-3 3.62E-4 GW 3181 -1.913E-2 -1.052E-2 1.573E-3 -1.941E-2 -1.148E-2 2.993E-3 3.62E-4 GW 3191 -1.941E-2 -1.148E-2 2.993E-3 -1.999E-2 -1.269E-2 4.119E-3 3.62E-4 GW 3201 -1.999E-2 -1.269E-2 4.119E-3 -2.076E-2 -1.411E-2 4.842E-3 3.62E-4 GW 3211 -2.076E-2 -1.411E-2 4.842E-3 -2.158E-2 -1.568E-2 5.091E-3 3.62E-4 GW 3221 -2.158E-2 -1.568E-2 5.091E-3 -2.232E-2 -1.731E-2 4.842E-3 3.62E-4 GW 323 1 -2.232E-2 -1.731E-2 4.842E-3 -2.286E-2 -1.891E-2 4.119E-3 3.62E-4 GW 3241 -2.286E-2 -1.891E-2 4.119E-3 -2.310E-2 -2.036E-2 2.993E-3 3.62E-4 GW 3251 -2.310E-2 -2.036E-2 2.993E-3 -2.297E-2 -2.157E-2 1.573E-3 3.62E-4 GW 3261 -2.297E-2 -2.157E-2 1.573E-3 -2.246E-2 -2.246E-2 -1.562E-18 3.62E-4 GW 327 -2.246E-2 -2.246E-2 -1.562E-18 -2.157E-2 -2.297E-2 -1.973E-3 3.62E-4 GW 3281 -2.157E-2 -2.297E-2 -1.973E-3 -2.036E-2 -2.310E-2 -3.753E-3 3.62E-4 GW 3291 -2.036E-2 -2.310E-2 -3.753E-3 -1.891E-2 -2.286E-2 -5.165E-3 3.62E-4 GW 3301 -1.891E-2 -2.286E-2 -5.165E-3 -1.731E-2 -2.232E-2 -6.072E-3 3.62E-4 GW $3311-1.731 \mathrm{E}-2$-2.232E-2 -6.072E-3 -1.568E-2 -2.158E-2 -6.384E-3 3.62E-4 GW 3321 -1.568E-2 -2.158E-2 -6.384E-3 -1.411E-2 -2.076E-2 -6.072E-3 3.62E-4 GW 3331 -1.411E-2 -2.076E-2 -6.072E-3 -1.269E-2 -1.999E-2 -5.165E-3 3.62E-4 GW $3341-1.269 \mathrm{E}-2$-1.999E-2 -5.165E-3 -1.148E-2 -1.941E-2 -3.753E-3 3.62E-4 GW 3351 -1.148E-2 -1.941E-2 -3.753E-3 -1.052E-2 -1.913E-2 -1.973E-3 3.62E-4 GW 3361 -1.052E-2 -1.913E-2 -1.973E-3 -9.796E-3 -1.923E-2 -1.715E-17 3.62E-4 GW 337 - 1 -9.796E-3 -1.923E-2 -1.715E-17 -9.294E-3 -1.975E-2 1.573E-3 3.62E-4 GW 338 1 -9.294E-3 -1.975E-2 1.573E-3 -8.956E-3 -2.070E-2 2.993E-3 3.62E-4 GW 3391 -8.956E-3 -2.070E-2 2.993E-3 -8.716E-3 -2.201E-2 4.119E-3 3.62E-4 GW 3401 -8.716E-3 -2.201E-2 4.119E-3 -8.501E-3 -2.361E-2 4.842E-3 3.62E-4 GW 3411 -8.501E-3 -2.361E-2 4.842E-3 -8.241E-3 -2.536E-2 5.091E-3 3.62E-4 GW 3421 -8.241E-3 -2.536E-2 5.091E-3 -7.880E-3 -2.712E-2 4.842E-3 3.62E-4 GW 343 1 -7.880E-3 -2.712E-2 4.842E-3 -7.377E-3 -2.873E-2 4.119E-3 3.62E-4 GW 344 1 -7.377E-3 -2.873E-2 4.119E-3 -6.716E-3 -3.005E-2 2.993E-3 3.62E-4 GW 345 - 1 -6.716E-3 -3.005E-2 2.993E-3 -5.905E-3 -3.095E-2 1.573E-3 3.62E-4 GW 3461 -5.905E-3 -3.095E-2 1.573E-3 -4.969E-3 -3.137E-2 -3.152E-19 3.62E-4 
GW 3471 -4.969E-3 -3.137E-2 -3.152E-19 -3.950E-3 -3.126E-2 -1.973E-3 3.62E-4 GW 3481 -3.950E-3 -3.126E-2 -1.973E-3 -2.898E-3 -3.065E-2 -3.753E-3 3.62E-4 GW 3491 -2.898E-3 -3.065E-2 -3.753E-3 -1.863E-3 -2.960E-2 -5.165E-3 3.62E-4 GW 3501 -1.863E-3 -2.960E-2 -5.165E-3 -8.871E-4 -2.823E-2 -6.072E-3 3.62E-4 GW 3511 -8.871E-4 -2.823E-2 -6.072E-3 -4.899E-18 -2.667E-2 -6.384E-3 3.62E-4 GW 3521 -4.899E-18 -2.667E-2 -6.384E-3 7.883E-4 -2.508E-2 -6.072E-3 3.62E-4 GW 3531 7.883E-4 -2.508E-2 -6.072E-3 1.487E-3 -2.363E-2 -5.165E-3 3.62E-4 GW 3541 1.487E-3 -2.363E-2 -5.165E-3 2.122E-3 -2.245E-2 -3.753E-3 3.62E-4 GW 3551 2.122E-3 -2.245E-2 -3.753E-3 2.736E-3 -2.166E-2 -1.973E-3 3.62E-4 GW $35612.736 \mathrm{E}-3$-2.166E-2 -1.973E-3 3.376E-3 -2.131E-2 -1.840E-17 3.62E-4 GW 3571 3.376E-3 -2.131E-2 -1.840E-17 4.090E-3 -2.144E-2 1.573E-3 3.62E-4 GW 3581 4.090E-3 -2.144E-2 1.573E-3 4.919E-3 -2.201E-2 2.993E-3 3.62E-4 GW 3591 4.919E-3 -2.201E-2 2.993E-3 5.888E-3 -2.293E-2 4.119E-3 3.62E-4 GW 3601 5.888E-3 -2.293E-2 4.119E-3 7.002E-3 -2.410E-2 4.842E-3 3.62E-4 GW 3611 7.002E-3 -2.410E-2 4.842E-3 8.241E-3 -2.536E-2 5.091E-3 3.62E-4 GW 3621 8.241E-3 -2.536E-2 5.091E-3 9.567E-3 -2.657E-2 4.842E-3 3.62E-4 GW 3631 9.567E-3 -2.657E-2 4.842E-3 1.092E-2 -2.758E-2 4.119E-3 3.62E-4 GW 3641 1.092E-2 -2.758E-2 4.119E-3 1.223E-2 -2.826E-2 2.993E-3 3.62E-4 GW 3651 1.223E-2 -2.826E-2 2.993E-3 1.342E-2 -2.851E-2 1.573E-3 3.62E-4 GW 3661 1.342E-2 -2.851E-2 1.573E-3 1.442E-2 -2.830E-2 9.318E-19 3.62E-4 GW 3671 1.442E-2 -2.830E-2 9.318E-19 1.518E-2 -2.761E-2 -1.973E-3 3.62E-4 GW 3681 1.518E-2 -2.761E-2 -1.973E-3 1.567E-2 -2.650E-2 -3.753E-3 3.62E-4 GW 3691 1.567E-2 -2.650E-2 -3.753E-3 1.589E-2 -2.505E-2 -5.165E-3 3.62E-4 GW 3701 1.589E-2 -2.505E-2 -5.165E-3 1.588E-2 -2.336E-2 -6.072E-3 3.62E-4 GW 3711 1.588E-2 -2.336E-2 -6.072E-3 1.568E-2 -2.158E-2 -6.384E-3 3.62E-4 GW 3721 1.568E-2 -2.158E-2 -6.384E-3 1.538E-2 -1.983E-2 -6.072E-3 3.62E-4 GW 3731 1.538E-2 -1.983E-2 -6.072E-3 1.509E-2 -1.824E-2 -5.165E-3 3.62E-4 GW 3741 1.509E-2 -1.824E-2 -5.165E-3 1.491E-2 -1.692E-2 -3.753E-3 3.62E-4 GW 3751 1.491E-2 -1.692E-2 -3.753E-3 1.494E-2 -1.591E-2 -1.973E-3 3.62E-4 GW 3761 1.494E-2 -1.591E-2 -1.973E-3 1.526E-2 -1.526E-2 -1.964E-17 3.62E-4 GW 3771 1.526E-2 -1.526E-2 -1.964E-17 1.591E-2 -1.494E-2 1.573E-3 3.62E-4 GW 3781 1.591E-2 -1.494E-2 1.573E-3 1.692E-2 -1.491E-2 2.993E-3 3.62E-4 
GW 3791 1.692E-2 -1.491E-2 2.993E-3 1.824E-2 -1.509E-2 4.119E-3 3.62E-4 GW 3801 1.824E-2 -1.509E-2 4.119E-3 1.983E-2 -1.538E-2 4.842E-3 3.62E-4 GW 3811 1.983E-2 -1.538E-2 4.842E-3 2.158E-2 -1.568E-2 5.091E-3 3.62E-4 GW 3821 2.158E-2 -1.568E-2 5.091E-3 2.336E-2 -1.588E-2 4.842E-3 3.62E-4 GW 3831 2.336E-2 -1.588E-2 4.842E-3 2.505E-2 -1.589E-2 4.119E-3 3.62E-4 GW 3841 2.505E-2 -1.589E-2 4.119E-3 2.650E-2 -1.567E-2 2.993E-3 3.62E-4 GW 3851 2.650E-2 -1.567E-2 2.993E-3 2.761E-2 -1.518E-2 1.573E-3 3.62E-4 GW 3861 2.761E-2 -1.518E-2 1.573E-3 2.830E-2 -1.442E-2 2.179E-18 3.62E-4 GW 387 1 2.830E-2 -1.442E-2 2.179E-18 2.851E-2 -1.342E-2 -1.973E-3 3.62E-4 GW $38812.851 \mathrm{E}-2$-1.342E-2 -1.973E-3 2.826E-2 -1.223E-2 -3.753E-3 3.62E-4 GW $38912.826 \mathrm{E}-2$-1.223E-2 -3.753E-3 2.758E-2 -1.092E-2 -5.165E-3 3.62E-4 GW 3901 2.758E-2 -1.092E-2 -5.165E-3 2.657E-2 -9.567E-3 -6.072E-3 3.62E-4 GW 3911 2.657E-2 -9.567E-3 -6.072E-3 2.536E-2 -8.241E-3 -6.384E-3 3.62E-4 GW $39212.536 \mathrm{E}-2$-8.241E-3 -6.384E-3 2.410E-2 -7.002E-3 -6.072E-3 3.62E-4 GW 3931 2.410E-2 -7.002E-3 -6.072E-3 2.293E-2 -5.888E-3 -5.165E-3 3.62E-4 GW 3941 2.293E-2 -5.888E-3 -5.165E-3 2.201E-2 -4.919E-3 -3.753E-3 3.62E-4 GW 3951 2.201E-2 -4.919E-3 -3.753E-3 2.144E-2 -4.090E-3 -1.973E-3 3.62E-4 GW 3961 2.144E-2 -4.090E-3 -1.973E-3 2.131E-2 -3.376E-3 -2.089E-17 3.62E-4 GW 397 1 2.131E-2 -3.376E-3 -2.089E-17 2.166E-2 -2.736E-3 1.573E-3 3.62E-4 GW 3981 2.166E-2 -2.736E-3 1.573E-3 2.245E-2 -2.122E-3 2.993E-3 3.62E-4 GW 3991 2.245E-2 -2.122E-3 2.993E-3 2.363E-2 -1.487E-3 4.119E-3 3.62E-4 GW 4001 2.363E-2 -1.487E-3 4.119E-3 2.508E-2 -7.883E-4 4.842E-3 3.62E-4 GW $40112.508 \mathrm{E}-2$-7.883E-4 4.842E-3 2.587E-2 0.000E+0 5.091E-3 3.62E-4 GM 00000000.0056990000

SM 2020 4.5720E-1 -4.5720E-1 0 4.5720E-1 4.5720E-1 0

SC $00-4.5720 \mathrm{E}-1$ 4.5720E-1 0

GE 00

GN -1 0000.0000 .000

LD 5000 5.8E7

EX 01201.0000

FR $\begin{array}{llllllll}0 & 1 & 0 & 0 & 917.21 & 0\end{array}$

RP 01110010.00 .05 .05 .00 .0 
EN 0

2. The NEC Cards for Modeling the Vertical Dipole at $1 \mathbf{~ m m}$ above the $3 \mathrm{ft} \mathbf{x} 3 \mathrm{ft}$ Metal

Plate

GW 1130000.063750000 .07975 1.016E-3

GW $1150 \begin{array}{llllllll}5 & 0 & 0 & 0 & 0 & 0 & 0.06375 & 1.016 \mathrm{E}-3\end{array}$

GW $115000079750000.1435 \quad 1.016 \mathrm{E}-3$

GM $00 \begin{array}{llllllllllll}0 & 0 & 0 & 0 & 0 & 0 & 0.001 & 0 & 1 & 0 & 13\end{array}$

SM 2020 4.5720E-1 -4.5720E-1 0 4.5720E-1 4.5720E-1 0

SC $\quad 0 \quad 0 \quad-4.5720 \mathrm{E}-1 \quad 4.5720 \mathrm{E}-1 \quad 0$

GE 0

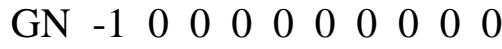

LD $5 \begin{array}{llllllllll} & 0 & 0 & 0 & 58000000 & 0 & 0 & 0 & 0 & 0\end{array}$

$\begin{array}{lllllllllll}\mathrm{EX} & 0 & 1 & 2 & 0 & 1 & 0 & 0 & 0 & 0 & 0\end{array}$

FR $\begin{array}{lllllllllll}0 & 1 & 0 & 0 & 971 & 0 & 0 & 0 & 0 & 0\end{array}$

RP 0011110010005500

$\mathrm{EN}$

3. The NEC Cards for Modeling the Horizontal Dipole at $1 \mathrm{~mm}$ above the $3 \mathrm{ft} \times$ 3ft Metal

\section{Plate}

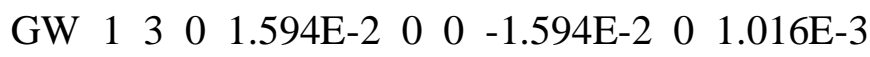

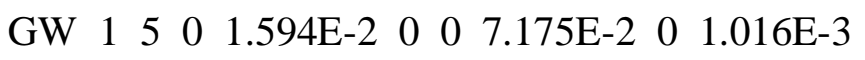

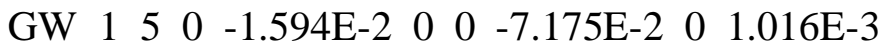

GM $00 \begin{array}{llllllllllllllll}0 & 0 & 0 & 0 & 0 & 0 & 0.002016 & 0 & 1 & 0 & 13\end{array}$

SM 2020 4.5720E-1 -4.5720E-1 0 4.5720E-1 4.5720E-1 0

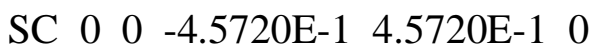

GE 0

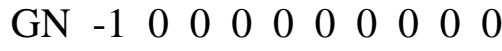

LD $5 \begin{array}{lllllllllll} & 0 & 0 & 0 & 58000000 & 0 & 0 & 0 & 0 & 0\end{array}$ 
EX $0 \begin{array}{llllllllllllll} & 1 & 2 & 0 & 1 & 0 & 0 & 0 & 0 & 0\end{array}$

FR $0 \begin{array}{llllllllll}0 & 1 & 0 & 0 & 971 & 0 & 0 & 0 & 0 & 0\end{array}$

RP $0 \begin{array}{lllllllllll}0 & 1 & 1 & 1001 & 0 & 0 & 5 & 5 & 0 & 0\end{array}$

EN

4. The NEC Cards for Modeling the Horizontal Loop at $1 \mathrm{~mm}$ above the $3 \mathrm{ft} \times$ 3ft Metal

Plate

GA 130.04978 -5 5 5.206E-4

GA $4250.049785355 \quad 5.206 \mathrm{E}-4$

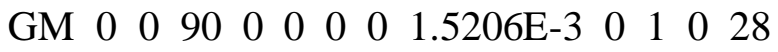

SM 2020 4.5720E-1 $-4.5720 \mathrm{E}-1 \quad 0 \quad 4.5720 \mathrm{E}-1 \quad 4.5720 \mathrm{E}-1 \quad 0$

$\begin{array}{lllllll}S C & 0 & 0 & -4.5720 \mathrm{E}-1 & 4.5720 \mathrm{E}-1 & 0\end{array}$

GE 0

GN $-1 \quad 0 \quad 0 \quad 0 \quad 0 \quad 0 \quad 0 \quad 0 \quad 0 \quad 0$

LD $\begin{array}{llllllllllll}5 & 0 & 0 & 0 & 58000000 & 0 & 0 & 0 & 0 & 0\end{array}$

EX $\begin{array}{llllllllll}0 & 1 & 2 & 0 & 1 & 0 & 0 & 0 & 0 & 0\end{array}$

FR $0 \begin{array}{llllllllll} & 1 & 0 & 0 & 1035 & 0 & 0 & 0 & 0 & 0\end{array}$

RP $0 \begin{array}{llllllllll}0 & 1 & 1 & 1001 & 0 & 0 & 5 & 5 & 0 & 0\end{array}$

EN 


\section{Appendix B Plain Surface Generator}

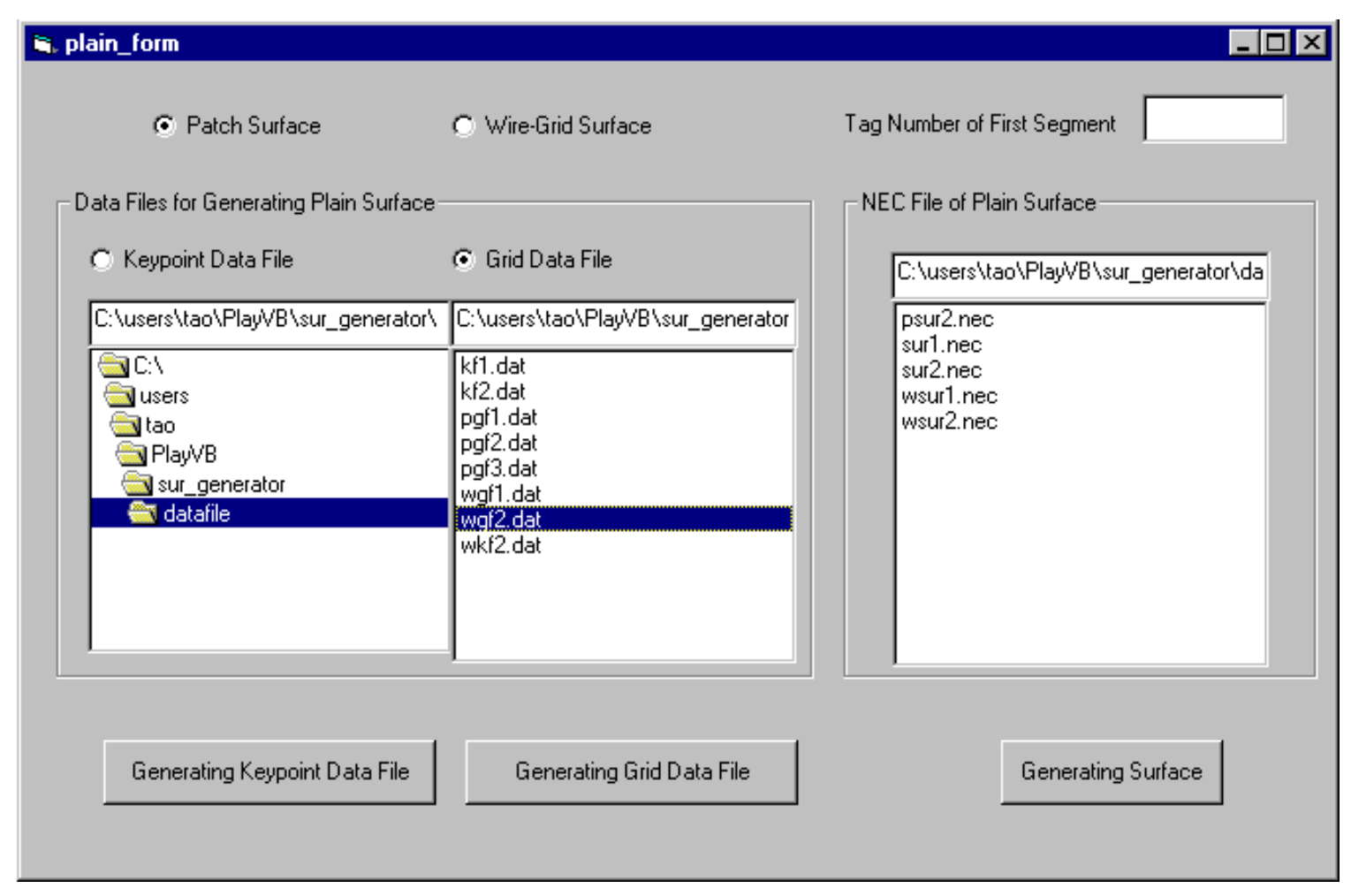

Figure 34 Plain_form

This form is used when the keypoint data file and the grid data file have been prepared. Two kinds of surfaces can be constructed; one is a patch surface, and the other is a wire-grid surface. For a wire-grid surface, the tag number of the first wire segment can be defined by user. With the keypoint data file and grid data file defined, the NEC file for plain surfaces could be generated. 


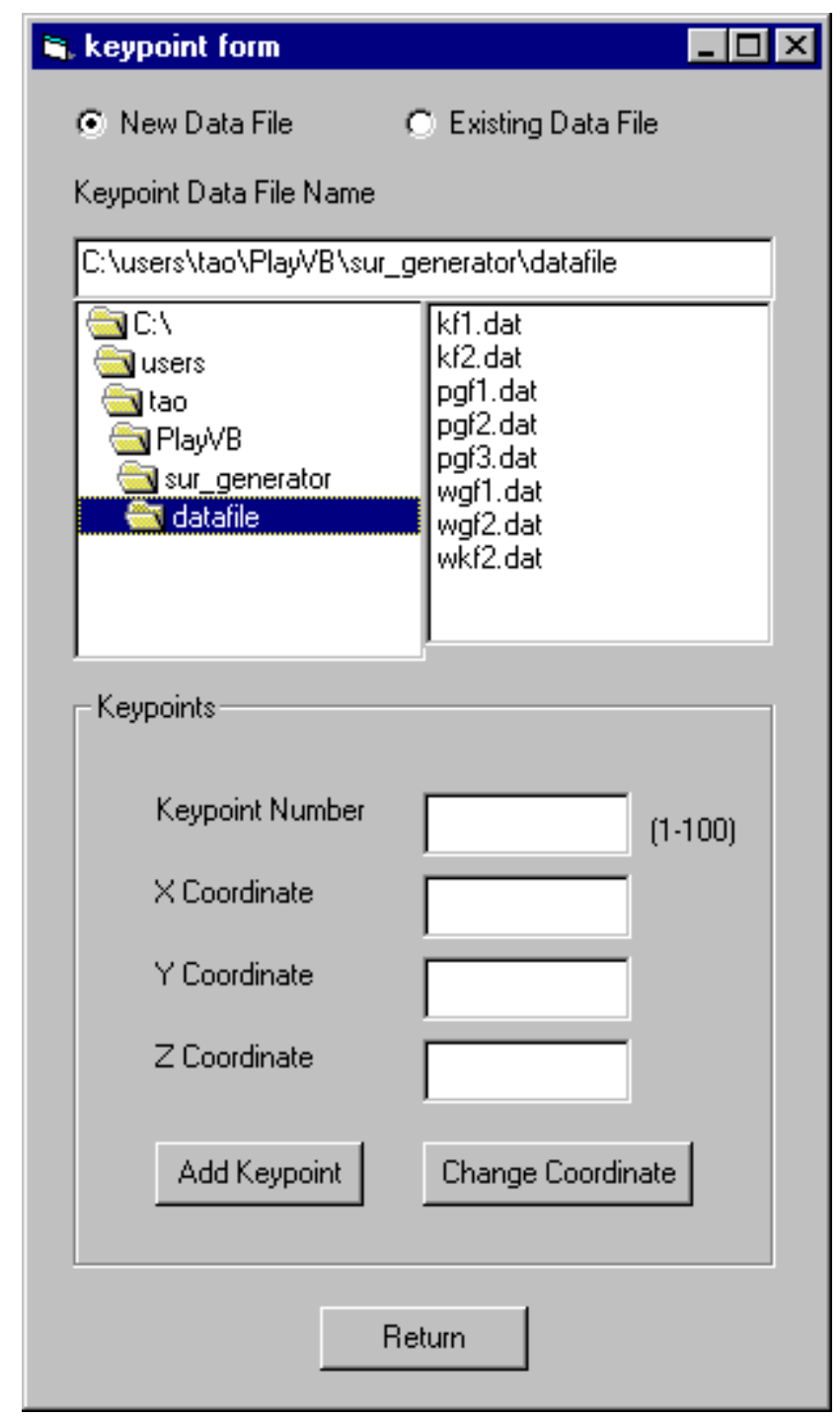

Figure 35 Form of Keypoint Data File Generator

The keypoint data file generator is used to generate a keypoint data file, which includes the total number of keypoints for constructing the surfaces, the keypoint number of each point, and its Cartesian coordinates (in meters). Keypoints are the corner points of each piece of a plain surface. Four keypoints are needed for each quadrilateral surface, and three keypoints are needed for each triangular surface. 


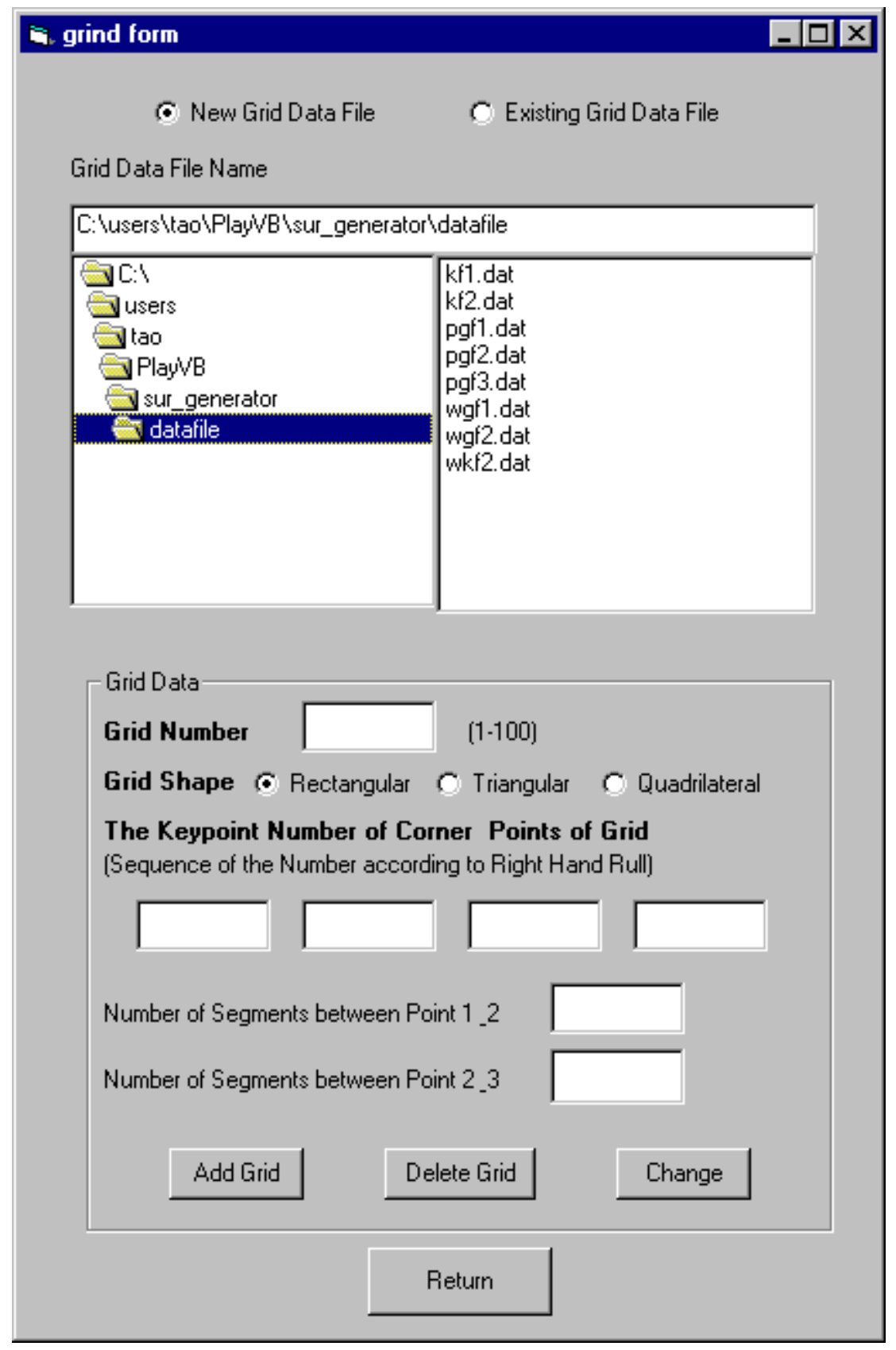

Figure 36 Form of Grid Data File Generator

The grid data file generator is used to generate a grid data file, which includes the total number of surface grids, the grid number of each grid, the shape of the grid (shape information will appear only in the grid data file of a patch surface), the keypoint numbers of the four/three keypoints of the grid, and the number of segments on each side of the grid (for a patch surface, 
only 1 segment is allowed for a triangular grid). For a patch surface, the normal vector of each grid is very important. It should direct outward for closed surfaces. The normal vector direction is determined by the ordering of the keypoints and the right-hand rule. For a wire-grid surface, the keypoints number should also be input in order. 


\section{Appendix C Properties of RANTEC FerroSorb}

- Rantec ${ }^{*}$ Ferrosob is fire retardant and meets the standards of Navel Research Laboratory Report 8093, Texas Instruments Report \#269066, and MIT MS-8-21.

Rantec Ferrosorb is tested in a non-destructive process using a coaxial reflectometer or an NRL arch. This process assures quality and performance, alkowing us to guarantee Rantec Ferrosorb's integrity.

\section{Electrical Properties}

\begin{tabular}{|c|c|c|}
\hline $\begin{array}{l}\text { FREQUENQY } \\
\text { RANGE }\end{array}$ & $\begin{array}{c}\text { ABS0RETJDW' } \\
\text { REFLEETWITY } \\
\text { AVERAGE }\end{array}$ & $\begin{array}{l}\text { PQWER HANDLING } \\
\text { COKTINUDUS GW }\end{array}$ \\
\hline $30 \mathrm{MHz}-40 \mathrm{GHz}$ & $\begin{array}{c}20 \mathrm{~dB} \text { oyer } \\
30 \mathrm{MHz}=40 \mathrm{GHz}\end{array}$ & $260 \mathrm{~W} / \mathrm{m}$ \\
\hline
\end{tabular}

\section{Applications}

Rantec Ferrosorb's ultra broadband frequency range, high absorption and power handling capabillites are ideal for IEC 801-3, MIL-STD 461/462D, and ANSI C63.4. Rantec ${ }^{9}$ Ferrosorb can be used for new construction as well as upgracting the performance of existing rooms. Chamber performance is guaranteed when installed and tested by Rantec technicians / RF engineers.

\section{Physical Properties}

\begin{tabular}{|c|c|c|c|c|}
\hline $\begin{array}{l}\text { HEIGHif } \\
\text { DWEFHLL }\end{array}$ & $\begin{array}{l}\text { UNTा } \\
\text { SlzE }\end{array}$ & $\begin{array}{l}\text { NDMAMAL } \\
\text { WEAGHT }\end{array}$ & MOUWTJNG & $\begin{array}{l}\text { AATINGS: } \\
\text { FIRE } \\
\text { PETAROANT }\end{array}$ \\
\hline $\begin{array}{l}410 \mathrm{MH} \\
16.1 \mathrm{in} .\end{array}$ & $\begin{array}{l}60 \mathrm{~cm} \\
5 q u a r e \\
23.62 \text { in. }\end{array}$ & $\begin{array}{l}19 \mathrm{~kg} \\
42 \mathrm{lbs} .\end{array}$ & $\begin{array}{l}\text { Epoxy or } \\
\text { Mech. Plate }\end{array}$ & $\begin{array}{l}\text { NRL 8Dg3 } \\
\text { DN } 4102 \cdot 82\end{array}$ \\
\hline
\end{tabular}
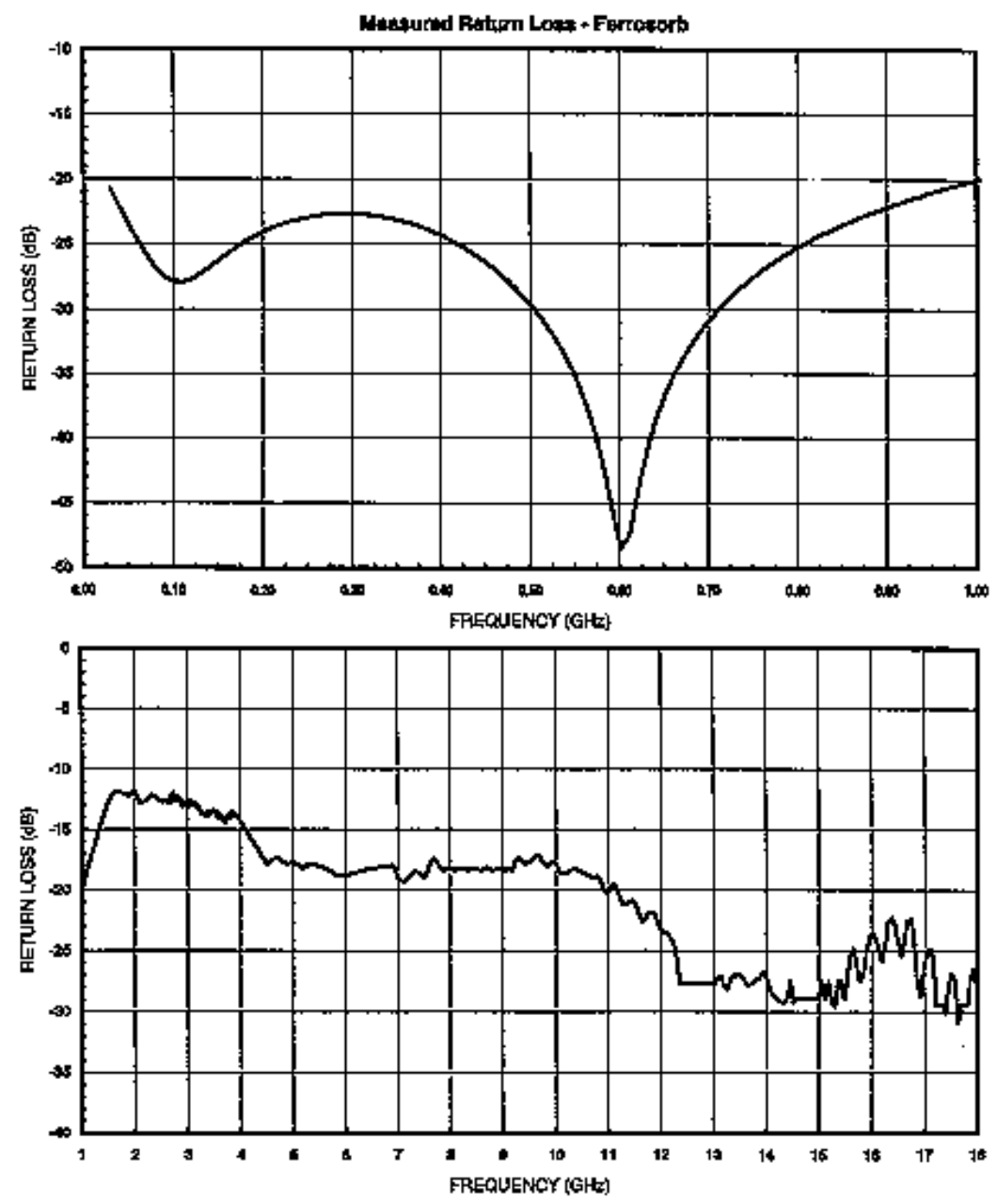


\section{Appendix D Specifications of HP8753D Network Analyzer}

\begin{tabular}{|c|c|}
\hline \multicolumn{2}{|c|}{$\begin{array}{l}\text { Test Port Output Characteristics } \\
\text { Prequency Chatracteristics }\end{array}$} \\
\hline Range: & $\begin{array}{l}300 \mathrm{kHz} \text { to } 3 \mathrm{Gllz} \\
30 \mathrm{kHz} \text { to } 6 \mathrm{GHz} \text { (with ojption } 0016 \text { ) }\end{array}$ \\
\hline \multirow[t]{2}{*}{$\begin{array}{l}\text { Resolution: } \\
\text { Stability: }\end{array}$} & $\begin{array}{l}1 \mathrm{~Hz} \\
\text { typically } \pm 7.5 \mathrm{sp}, \mathrm{m} 0^{\circ} \text { to } 55^{\circ} \mathrm{C} \\
\text { typically } \pm 3 \mathrm{ppm} / \mathrm{yes}^{\prime}\end{array}$ \\
\hline & $\begin{array}{l}\text { With Oftion 105: } \\
\text { typically } \pm 0.05 \text { ppm } 0^{\circ} \text { to } 55^{\circ} \mathrm{C} \\
\text { typically } \pm 0.5 \text { ppmiysur }\end{array}$ \\
\hline Aceuracy: & \pm 10 ppm at $25^{\circ} \mathrm{C} \pm 5^{\circ} \mathrm{C}$ \\
\hline Yower танg: & $\begin{array}{l}-5 \text { to }+20 \text { dBw } \\
-5 \text { to }+18 \text { dBan (with Option } 006 \text { ) }\end{array}$ \\
\hline Resolution: & $0,05 \mathrm{~dB}$ \\
\hline Levol acturucy": & $\doteq 1.0 \mathrm{~dB}$ \\
\hline Level linearity ${ }^{3.2 .2 s_{t}}$ & $\begin{array}{l}(-5 \mathrm{dBm} \omega+15 \mathrm{dBm}): 0.25 \mathrm{~dB} \\
(+15 \mathrm{~dB} w 1 \mathrm{to}+20 \mathrm{dBm}): 1: 0.5 \mathrm{~dB}\end{array}$ \\
\hline Inıpedance: & 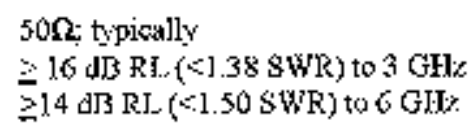 \\
\hline \multicolumn{2}{|l|}{ Spectral jurity: } \\
\hline 2nd harmonicic & 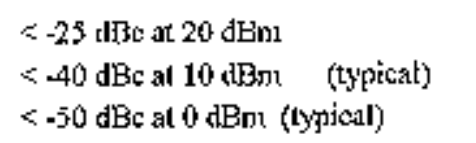 \\
\hline \multirow[t]{3}{*}{ 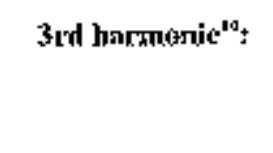 } & $<-25 \mathrm{dBc}$ at $20 \mathrm{dBm}$ \\
\hline & $<+10 \mathrm{dBc}$ at 10 dithn (tyoical) \\
\hline & $<-50$ dBo at 0 dHan (typical) \\
\hline \multicolumn{2}{|c|}{ Nonharmonic spurious: } \\
\hline Mixer related: & $\begin{array}{l}<-30 \mathrm{~dB} \text { at } 20 \mathrm{dBm} \text { (typical) } \\
<-55 \mathrm{~dB} \text { at } 0 \mathrm{dBm} \text { (typical) }\end{array}$ \\
\hline
\end{tabular}

\author{
Test Port Input Characteristics \\ Freguency range: \\ $300 \mathrm{kHz}$ to $3 \mathrm{GH} z$ \\ $30 \mathrm{kHz} /$ to 6 Gllt? (with Option 006 ) \\ Avernge roise level: \\ -90 dBm (3 kW BW, 50 kIfz o $3 \mathrm{GHz})$ \\ $-110 \mathrm{dBm}$ (10 IJz ISW, $50 \mathrm{kHz}$ to $3 \mathrm{GHz}$ ) \\ $-120 \mathrm{dBm}$ (10 IJz J3W, $50 \mathrm{kHz}$ to $3 \mathrm{GHz})$ \\ (typitual) \\ $-85 \mathrm{dBm}(3 \mathrm{kJHz} \mathrm{JW}, 3$ to $6 \mathrm{GHz})$ \\ $-105 \mathrm{dBm}(10 \mathrm{H} \% \mathrm{BW}, 3$ to $6 \mathrm{GHz})$ \\ $-115 \mathrm{dBm}(10 \mathrm{k} / \mathrm{BW}, 3$ 10 $6 \mathrm{GHz}$ (typical) \\ Maxinıum input level: $\quad$ d $\mathrm{dBm}$ \\ Damage level: $\quad 20$ ABn1 or 33 VDKC \\ Impedance: 50 ohnz: $\geq 10 \mathrm{AB} \mathrm{RL}, 30 \mathrm{kLJz} 1050 \mathrm{kHz}$. \\ $\geq 20 \mathrm{AB} \mathrm{RL}, 50 \mathrm{kHz} 10,300 \mathrm{kHz}$ \\ $\geq 23 \mathrm{~dB}$ RL, $300 \mathrm{kHL}$ to $1,3 \mathrm{GHz}$ \\ $\geq 20 \mathrm{~dB}$ RL. $1.3 \mathrm{GHz}$ to $3 \mathrm{GL}$ \\ $\geq 8 \mathrm{dBRL}, 3 \mathrm{GHz}$ to $6 \mathrm{GHL}$ \\ Frequency responses $\quad \pm 1.0 \mathrm{dL}, 300 \mathrm{kHz}$ to $3 \mathrm{GHz}$ \\ $\left(25^{\circ} \pm 5^{\circ} \mathrm{C}\right) \quad \pm 2.0 \mathrm{dE}, 3 \mathrm{GHz} 106 \mathrm{GHz}$ \\ Harmonics (Option 002):

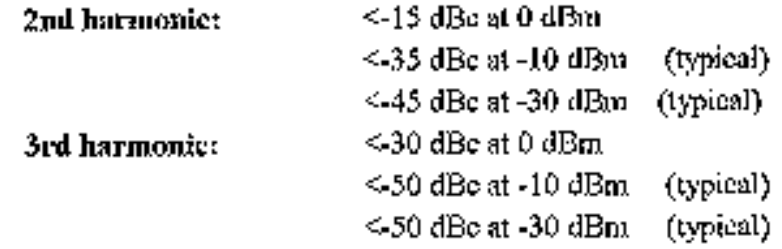 \\ Harmonic Measurement kecuracy: $\left(25^{\circ}, 5^{\ominus} \mathrm{C}\right)$ \\ $16 \mathrm{MHz}$ to $3 \mathrm{GHz} \quad \pm 1 \mathrm{~dB}$ \\ $3 \mathrm{GHz}$ to $6 \mathrm{GHz}: \quad \pm 3 \mathrm{~dB}$ (with Option 006 ) \\ Harmunic Measuresutent Dymanic Range: \\ $-40 \mathrm{~dB}$ (oulput $=-10 \mathrm{dBm}$, icrput $<.15 \mathrm{dBm}$ )
}




\section{Approval of Examining Committee}

Roy S. Nutter, Ph.D.

Gregory J. Thompson, Ph.D.

James E. Smith, Ph.D., Chairman

Date 